\title{
ANALYTICAL APPLICATIONS OF CALIXARENES
}

\author{
Y.K. Agrawal, S. Kunji and S.K. Menon \\ Department of Chemistry \\ School of Sciences, Gujarat University \\ Ahmedabad, India
}

\section{CONTENTS}

Page

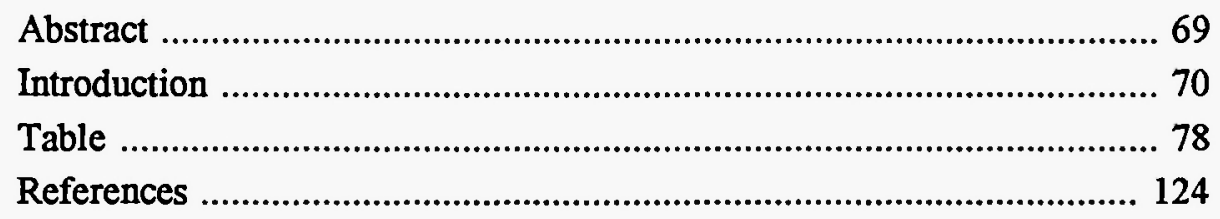

\section{ABSTRACT}

Calixarene chemistry is developing fast; in the present decade its status in supramolecular chemistry gives the scientific community a springboard to expand the applications of these cyclic oligomers into other areas of science. Though calixarenes have shown more of their molecular ability as ionophores, still a large part of their utility remains unexplored. In the present review we try to present the history of calixarenes by tracing the various stages of their development and their applications. 


\section{INTRODUCTION}

Calixarenes, a family of vase-like cyclic macrocycles, have been under development in the research laboratories for just over 50 years, with the first paper on calixarenes having appeared in $1944 / 1 /$. In the half century since their inception, calixarenes have evolved from a scientific curiosity to a topic truly useful in the utilitarian sense or just intriguing in the intellectual sense, obviously complemented by a group of versatile techniques capable of characterizing these macromolecules and assessing their potentiality. In the present decade emphasis was placed on their supramolecular properties because of their flexibility towards structural-molecular modifications which account for a hydrophobic cavity and the use of second sphere coordinations to assemble large aggregates.

The origin of calixarenes, however, can be traced back to Baeyer's discovery of phenol formaldehyde resins in the $1870 \mathrm{~s} / 2 /$. Baeyer, best known for his work on saturated carbocyclic ring compounds and synthesis and structural elucidation of naturally occurring dye indigo, examined compounds obtained by mixing aldehydes and phenols in the presence of strong acids $13,4 /$. Though failing to characterize the cement-like condensed product, Baeyer nevertheless gave to the chemical world "phenolformaldehyde chemistry". Investigation of these compounds was actively pursued by researchers like Lederer /5/, Manasse /6/, Blumer /7/ Storey /8/ and Luft /9/, who all tried to subdue these resinous tars and find their commercial applications but were unable to produce materials of marketable quality.

These compounds crossed the academic-industrial dividing line in 1907 when Baekeland /10-13/ filed a patent on his process to make Bakelite /14/. Both Baeyer and Baekeland $/ 2-4,10,11 /$ as well as Gills $/ 13 /$, realized that $\mathrm{CH}_{2}$ and $\mathrm{CH}_{2} \mathrm{OCH}_{2}$ groups are the most likely type of linkages between a pair of aromatic rings in a phenol-formaldehyde condensation product. Zinke and coworkers $/ 1,15-18 /$ simplified the problem by looking at p-substituted phenols instead of phenols because, unlike phenol which itself reacts at the ortho and para positions to form a highly cross-linked polymer, a para alkyl phenol on the contrary can react only at the two ortho positions, denying 
cross-linking possibilities. The product that Zinke et al. obtained in their first experiment $/ 17 /$ was a crystalline acetate (m.p. $314^{\circ} \mathrm{C}$ ) and it had a surprisingly high molecular weight of 1725 . Zinke $/ 16 /$ proposed a cyclic tetrameric structure for his product in accordance with Niederl and Vogel /19/, who had also proposed a cyclic structure for compounds obtained by acid-catalysed treatment of aldehydes and phenols. In contrast to Zinke tetrols, wherein the four $\mathrm{OH}$ groups were intrannular, the Niederl octols had eight extrannular $\mathrm{OH}$ groups $/ 20 /$.

Abundant 'new evidence accumulated to substantiate the structure and synthesis of these compounds when the further four papers published by Zinke $/ 15-18 /$ described cyclic tetramer prepared from p-methyl-, p-tert butyl-, p-tert pentyl-, p-(1,1,3,3-tetra methyl)-, p-cyclo hexyl-, p-phenyl- and p-benzyl phenol. Zinke /16/ assigned tetrameric structure to these macrocycles and called them "mehrkernmethylenephenolverbindungen" (polynuclear methylenephenol compounds) $/ 15 /$.

The other method, first worked out by Hayes and Hunter $/ 21 /$ in 1952 and exploited and improved a few years later by Kämmerer et al. /22-29/ involves the sequential addition of methylene groups and aryl functions to a p-substituted phenol blocked at one of the ortho positions by a halogen. A linear oligomer can be elaborated, which carries a hydroxymethyl group at one end and the halogen blocking group at the other end. Removal of the halogen by hydrogenolysis, followed by acid-catalyzed high dilution cyclization, gives the cyclic oligomer. This method demonstrated its potential in the preparation of a series of methyl 4-tert butyl substituted calixarenes, including cyclic tetramers, pentamers, hexamers and heptamers. Molecular weight determination, melting point, elemental analysis and ultraviolet spectra were consistent with a cyclic tetramer.

Cornforth $/ 30 /$ ventured into the Zinke procedure and negated the tacit assumption that a single product is formed in every instance. He used p-tert butyl phenol as the starting material and isolated two materials, both crystalline, having high but non-identical melting points. When $\mathrm{p}$-(1,1,3,3-tetra methyl butyl) phenol was used as the starting material, again two compounds were isolated. Cornforth inferred that both compounds were diastereoisomers arising from hindered rotation. However, this interpretation was disproved 
when Kämmerer et al. $/ 22 /$ and John Munch /31/ of the Petrolite Corporation (St. Louis) produced data on 'HNMR studies which showed rapid conformational interconversion occurring at room temperature. Buriks et al. /32,33/ investigated the petrolite demulsifier made by oxyalkylating p-tert butyl phenol and formaldehyde and concluded that, although the synthetic route followed was different from the one described by Zinke, the material they isolated must be Zinke's cyclic tetramer.

Gutsche /34-38/, a pioneer in calixarene chemistry, explored Zinke's compounds and accredited them as potential candidates for enzyme mimic building. He coined the word "calixarenes" in 1975 /35/, with 'calix' meaning vase or chalice and 'arene' indicating the number of aryl moieties in the macrocyclic array. He further used different substituted phenols, viz., p-methyl-, p-tert butyl-, p-phenyl-, p-methoxy- and p-carboxy methoxy phenol and elucidated the condensation products as cyclic tetramers $134 /$. However, Gutsche demonstrated that the outcome of the reactions can be strongly influenced by reaction conditions, thereby showing the synthesis of calixarenes with $n=4-8$. All structures are in complete agreement with chemical, spectral and analytical data, further definitely destablished by single crystal $\mathrm{X}$-ray crystallography by Andreetti, Ungaro and Pochini 139-41/.

During the decade that followed, calixarene chemistry progressed steadily in the academic laboratories, ultimately becoming accessible to a wider community of research. Attention is presently focused on ways in which the baskets have been or might be put to use with change in structural-molecular properties, and their promise as selective and useful complexation agents.

Several methods are in practice for attaching the calixarenes with functional groups. These involve:

reactions at the lower rim of the calix:

viz., ester formation with monofunctional reagents, ether formation with monofunctional reagents and esterification/etherification with polyfunctional reagents. 
reactions at the upper rim of the calix:

viz., dealkylation of p-substituted calixarenes, functionalization via $\mathrm{p}$-Claisen rearrangement route, $\mathrm{p}$-quinonemethide route and $\mathrm{p}$-chloromethylation route.

\section{oxidation reaction of calixarenes:}

viz., oxidation of the methylene bridges and oxidation of the aryl rings.

The phenol derived calixarenes cany hydroxyl groups at their lower rim and they provide sites for attaching other functional groups. When an excess of acylating agent is used, generally all the hydroxyl groups are converted into ester groups $/ 42 /$. Partial acylation $/ 43,44 /$ occurs under certain conditions, for example a pair of acetates that are not conformational isomers can be isolated from the sulfuric acid catalysed reaction of acetic anhydride and tert-butyl calix(4)arene. Calix(6)arene and calix(8)arene on acid catalysed acylations yield hexaacetoxy and octaacetoxy compounds respectively $/ 36 /$. Benzoylation $/ 45 /$ of calix(6)arene has been reported to yield the hexabenzoate as a solid melting above $400^{\circ} \mathrm{C}$.

By etherification methyl, ethyl, allyl and benzyl ethers have all been prepared with high yields /44/. Treatment of calixarene with an alkyl halide in THF-DMF solution in the presence of sodium hydride is reported. Cornforth et al. 130 / have synthesized a series of polyalkoxy ethers by using tosylate of the alkylating agent in the presence of potassium tert-butoxide 146/. Other reagents like diazomethane $/ 47 /$, benzyl tosylate, dimethyl sulfate 144/, hexamethyl disilazene, N,O-bis (trimethylsilyl) acetamide and chloromethyl silane effect etherification.

The theme of using ether/ester formation to introduce functionalization has been exploited by Arduini et al. /48/. They prepared the first water soluble calixarenes (soluble to the extent of ca. $10^{-4} \mathrm{M}$ ) by preparing a tetraacid from tetraester by base induced hydrolysis. A diacid was also obtained from diester using potassium tert-butoxide rather than $\mathrm{NaH}$ in the former reaction /49/. Ungaro et al. carried out this same procedure on a lower rim bridged calixarene to produce the crown 5-diacetic acid. Later on Ungaro, 
Mckervey et al. and others /41-54/ reported various structural combinations. Several patents were filed because of the commercial interest in these compounds.

The selective removal of the tert-butyl groups from dimethyl di tert-butyl calix(4)arene is adequately described by Böhmer et al. $155 /$. The procedure is now extensively used as a means for obtaining calixarenes containing unsubstituted p-positions, thereafter making the amenable to functionalization, for example calix(4)arene 156,57/ (yield: 65-70\%), calix(6)arene $/ 45 /$ (yield: $89 \%$ ), and calix(8)arene $/ 45$ / (yield: $93 \%$ ) were synthesized following this procedure.

p-sulfonato calix(6)arene $/ 58,59 /$ and p-nitro calix(6)arene /60,61/ can be prepared by functionalization via electrophilic substitution or direct nitration to get nitrocalix(6)arenes $/ 62,63 /$.

One of the most productive reactions involves the p-Claisen rearrangement in which calix(4)arene is readily converted into tetra allyl ether, which on refluxing with $\mathrm{N}, \mathrm{N}^{\prime}$-diethyl aniline produces p-allyl calix(4)arene, which is further converted to p-toluene sulfonate that can give a variety of functionalized calix(4)arenes. Another procedure for direct electrophilic substitution takes into account the nucleophilic character of the para positions of the phenolates $/ 62 /$. When calix(4)arene is mixed with formaldehyde along with the secondary amines, a Mannich type reaction occurs to produce alkyl amino methyl calix(4)arenes.

Another procedure for dealkylation via the p-quinone methide route provides a particularly short pathway to a variety of functionalized calixarenes, its importance being the introduction of carboxyl groups into the calixarene framework /63/. Functionalization of calixarenes has been further expanded by the p-chloromethylation route /64/, introduced by Arduini et al. to produce a number of $\mathrm{p}$-chloromethyl compounds.

Oxidation reactions of calixarenes include oxidation of the methylene bridges and oxidation of the aryl rings. Moshfegh et al. /65-67/ and Ninagawa et al. $/ 68 /$ have reported the action of chromic acid on ester of cyclic tetramers, cyclic hexamers and cyclic octamers with methylene groups converted to keto groups. Oxidation of aryl rings by Fremy's salt has been reported by Rosyk $/ 69 /$. 
Various functionalization possibilities give calixarenes good recognition in the list of complex forming macrocycles, with facets in both state and solution state complexation. Solution state complexation involves both aqueous and non-aqueous solvents. Calixarenes form endocalixarene complexes with aromatic molecules, viz. pyridine $/ 70 /$, chloroform $136,71,72 /$, benzene $/ 41,73,74 /$, xylene $/ 41$, anisole $/ 41 /$, toluene $/ 39,75-78 /$; non-aromatic guest molecules $/ 51,79 /$, viz., acetonitrile $/ 80 /$, cyclohexane 181 , isopropyl alcohol / $82 /$, acetic acid $/ 83 /$, acetone $/ 84 /$, sodium bromide $/ 80 /$, carbon disulfide $/ 85,86 /$, methanol $/ 36,87 /$, methylene chloride $/ 83 /$, methyl cyanide $189 /$; metal cation complexes $/ 43,49,53,90-99 /$; organic cations viz., amines $/ 100,101 /$ and neutral organic molecules $/ 102,103 /$, viz., glycerol, D-ribose, riboflavin, D-glucose, Vitamin $\mathrm{B}_{12}$, hemin and sugars. Attractive $\mathrm{CH}_{3} \ldots \pi$ interactions contribute to the binding which occurs between the protons of the tert-butyl groups and the aromatic ring of the guest molecule $/ 70,88 /$. Atwood $/ 104 /$ has reported an example of inclusion complexation of water by a calix(4)arene sulphonate. The tenacity with which the guest molecule is held varies widely within the series. The structures of complexed calixarenes and those of parent calixarenes are most effectively revealed by X-ray crystallography /105/. Another analytical technique that has been infrequently applied to molecular complexes is that of ${ }^{13} \mathrm{C}$ cross polarisation magic angle spinning NMR /106/. Elements like $\mathrm{S}, \mathrm{Cl}, \mathrm{Cs}$, As and $\mathrm{N}$ are captured in the cavities by joining a pair of cavitands, viz., upper rim to upper rim.

Calixarenes, as ionizable receptors, were first exploited by Izatt $e t$ al. $191,92 /$, who achieved selective transport of alkali cations from one aqueous phase to a chloroform membrane containing the p-tert butyl tetramer, hexamer or octamer. The octamer showed the fastest rate, with a cation selectivity order $\mathrm{Cs}>\mathrm{Rb}>\mathrm{K}>\mathrm{Na}>\mathrm{Li}$.

The p-tert butyl calix(8)arene can be deprotonated and this feature can be used for converting the lanthanides into complexes that are soluble in organic solvents $/ 107 /$, suggesting that it may be used in solvent extraction and purification of these elements. The complexation of alkali and alkaline earth cations by neutral ligands has been examined extensively by using phase transfer from water into an organic solvent, ion transport through liquid 
membranes and stability constant measurements. The ratio of the stability constants adequately expresses the selectivity of one cation relative to another. These values are important when behaviour of calixarenes is compared with crown ethers, cryptands and natural ionophores such as valinomycin and monensin. Shinkai et al. /108/ have found stability constants for $\mathrm{UO}_{2}^{+2}$ with calixarene derivatives up to $10^{19} \mathrm{~mol}^{-1}$ and selectivity factors of $10^{11}-10^{19}$ with respect to other divalent cations, viz., $\mathrm{Hg}^{2+}, \mathrm{Zn}^{2+}, \mathrm{Ni}^{2+}$ and $\mathrm{Cu}^{2+}$.

Crystal structures are now available for various complexes of calixarenes with metals including $\mathrm{Co}^{2+}, \mathrm{Fe}^{2+}, \mathrm{Ni}^{2+}$ and $\mathrm{Cu}^{2+}$. Calix(4)arene diquinone crown ether shows strong complexation and electrochemical recognition for $\mathrm{Na}^{+}, \mathrm{K}^{+}, \mathrm{Ba}^{2+}, \mathrm{NH}_{4}^{+}$and $\mathrm{BuNH}_{3}{ }^{+}$guests. The structures of three chromium complexes prepared from cone and 1,3-alternative 25,26,27,28-teta propoxy calix(4)arene and $\mathrm{Cr}(\mathrm{CO})_{3}$ were determined by single crystal X-ray studies /109/.

Recent developments involve studies on solid co-ordination compounds of calcium and cadmium with a p-tert butyl hydroxy calix(8)arene, extraction and solution thermodynamics of complexation of alkali and alkaline earth cations by calix(4)arene amides, conformational and binding properties of calix(6)arene based ester ligands, calixarene derivatives and their rare earth metal complexation, spectroscopic characterization of the interactions between calixarene ionophores and metal ion solutions, and so on.

With the horizon of complexation abilities and salient properties of calixarenes increasing, there is an increase in the number of reports, monographs and patents. Most of the patents feature calixarenes as an item of commerce. However, its varied applications and molecular structural properties shows calixarene chemistry as a subset of supramolecular chemistry /110/. One of the first applications was that of the Petrolite company in the US, who in 1950 marketed the product of o-alkylation of p-tert butyl phenol formaldehyde oligomers as a surfactant with excellent demulsifying properties. Most of the recent applications are associated with their properties as molecular receptors /91-92/. Izatt has used calixarenes for the recovery of cesium from nuclear waste. Calix(6)arene hexa acid "Super Uranophile" attached to polystyrene is applied for the recovery of uranium from sea 
water. A polymethacrylate with calix(4)arene esters spaced regularly about the backbone is capable of sequestering $\mathrm{Na}^{+}$selectively and may be useful in trans-membrane channels /110/.

European and U.S. patents /I11-114/ from Loctite Corporation contain claims on instant adhesive composition utilizing calixarene accelerators. Compositions for cyanoacrylate adhesives use calixarenes as additives which lead to an important decrease of fixture times and cure on deactivating substractions such as wood and leather. One of these patents /111/ claims calixarenes to be useful not only as ion-exchangers but also as phase transfer catalysts, coatings, adhesives and potting compounds. Ion selective field effect transistors (ISFET) containing a calixspherand as the ionophore enable the quantitative determination of $\mathrm{K}^{+}$in the presence of a large excess of $\mathrm{Na}^{+}$. Calix(6)arene bound to an ISE is used to determine $\mathrm{Pb}^{2+}, \mathrm{Ag}^{+}$and $\mathrm{Cr}^{2+}$ ions simultaneously by using differential pulse anodic stripping voltammetry. Bridged calixarenes are also possible, in which the structural features of calixarenes and spherands are combined to make receptors fixed in a very rigid binding conformation $/ 109,115 /$.

Chromatographic applications include using calixarenes as a stationary phase in gas chromatography for separating alcohols, chlorinated hydrocarbons and aromatic compounds /116/. Some calixspherand complexes exhibiting high thermodynamic and kinetic stability, suggesting possible applications, are beginning to emerge in medical diagnostics, sensors and chromatography /117/. A recent approach is to include the calixarene based sensor with several others in an array of sensors. Ion selective electrodes for $\mathrm{K}^{+}$and $\mathrm{Cs}^{+}$have been made using dioxocalix(4)arene and calix(6)arene respectively as ionophore /118/. PVC membranes along with calix(4)arene show remarkable selectivity. This material has been used to make bench and miniaturised $\mathrm{Na}$ selective electrodes $/ 119 \mathrm{a} /$ and a $\mathrm{Na}$ electrode for flow injection analysis (FIA) $/ 119 \mathrm{~b} /$. This approach gives very accurate assays for sodium and other ions in blood and mineral water $j 120 /$.

Table 1 presents an updated summary of complexometric studies on the various calixarenes. 

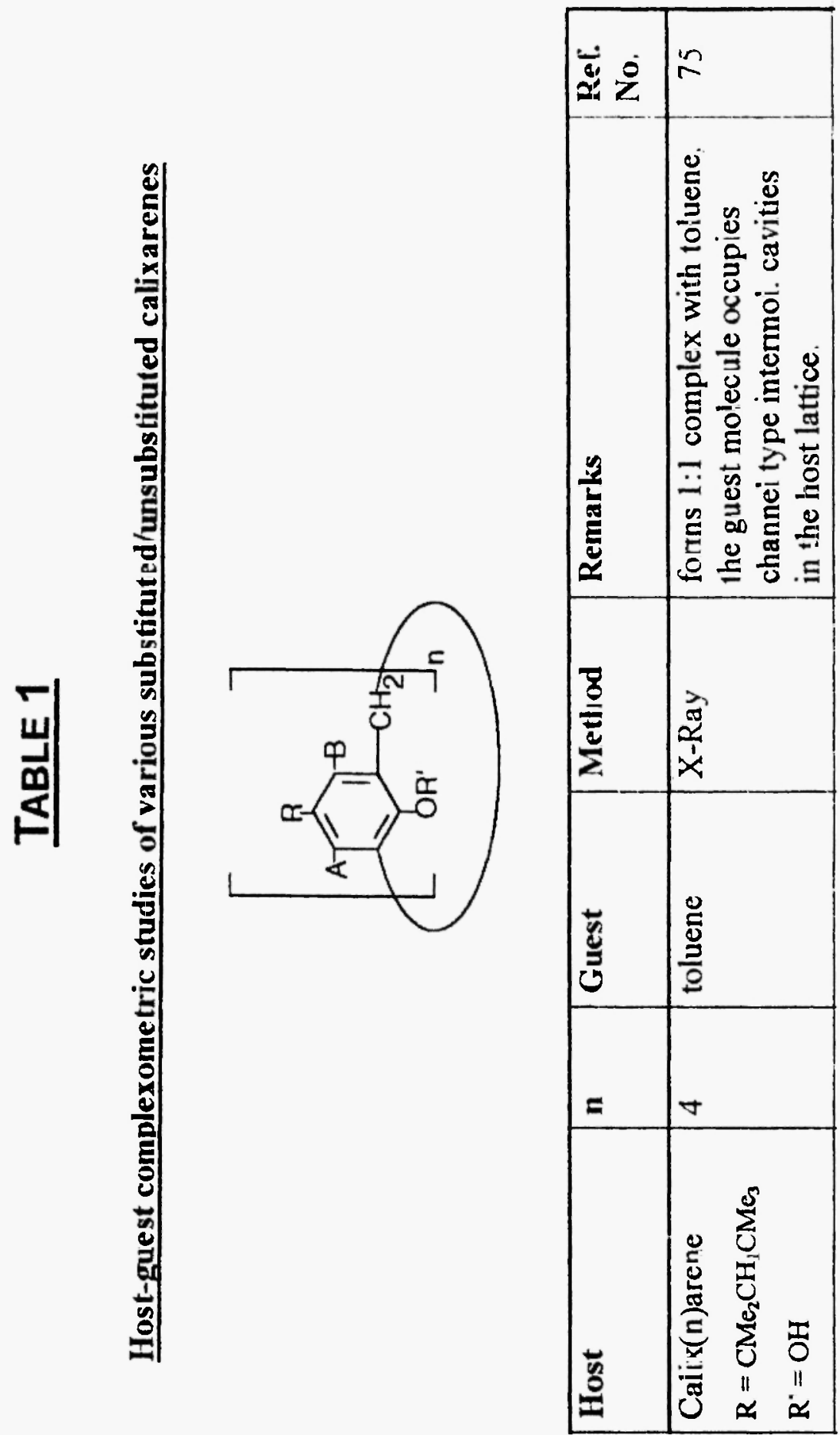


\begin{tabular}{|c|c|c|c|}
\hline$\cong$ & $\bar{\sim}$ & $\bar{n}$ & 18 \\
\hline 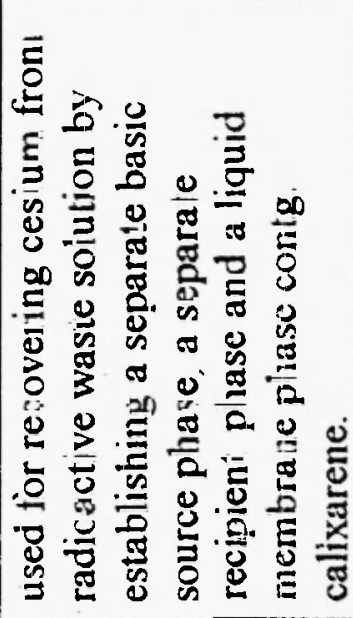 & 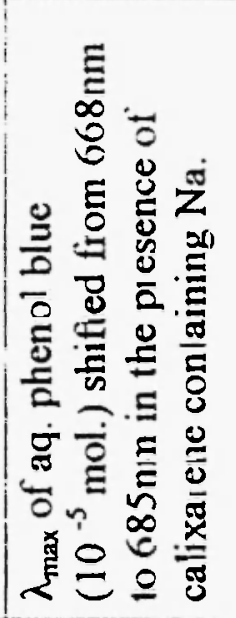 & 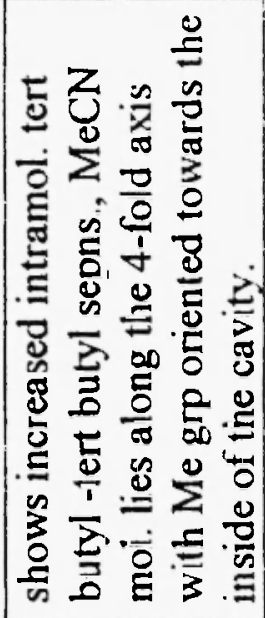 & 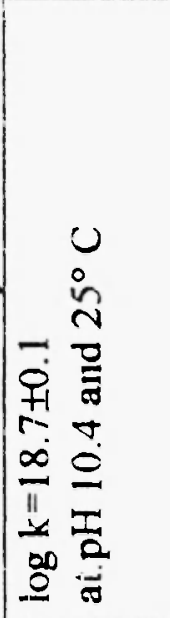 \\
\hline$\sum$ & 3 & 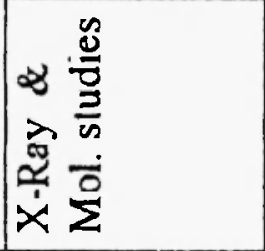 & 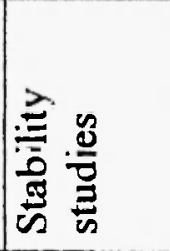 \\
\hline 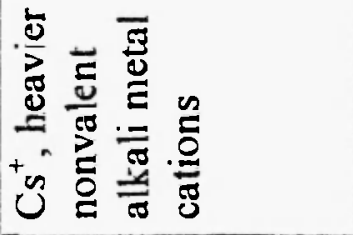 & $\left.\right|^{+}$ & Z & $\overbrace{}^{+}$ \\
\hline 1 & 10 & $\nabla$ & 0 \\
\hline 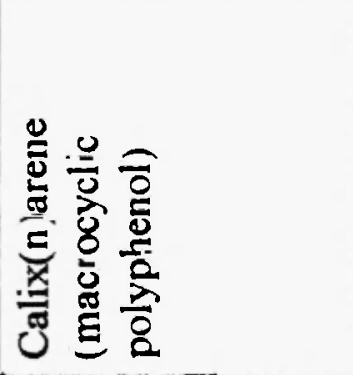 & 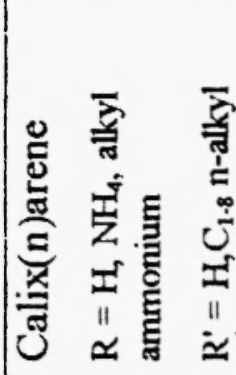 & 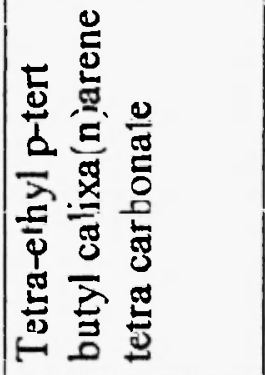 & 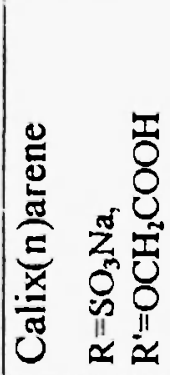 \\
\hline
\end{tabular}




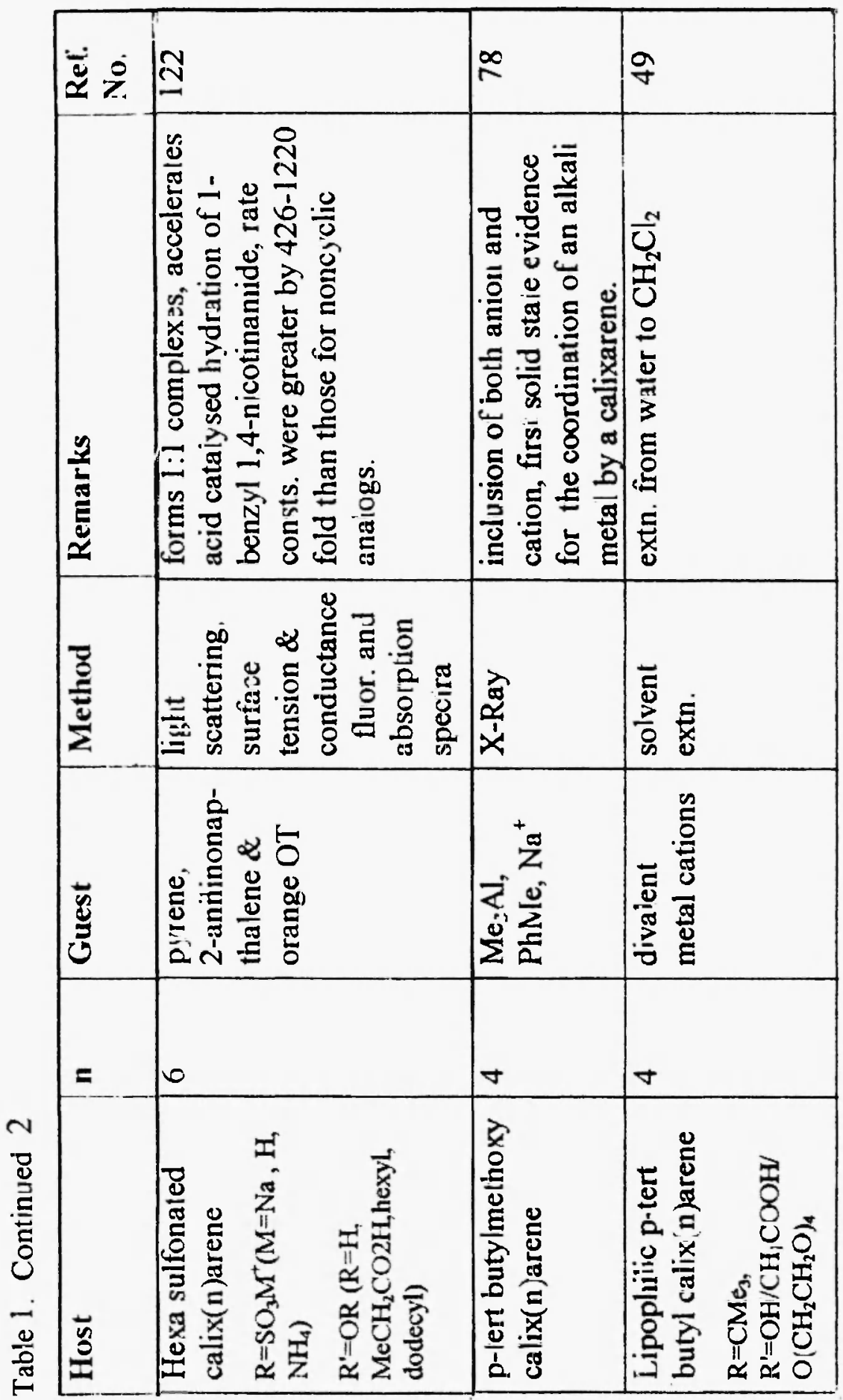




\begin{tabular}{|c|c|c|c|c|c|c|}
\hline$\stackrel{\Perp}{\approx}$ & $\stackrel{ \pm}{\beth}$ & $n$ & & $\stackrel{\sim}{\simeq}$ & 0 & $\pi$ \\
\hline 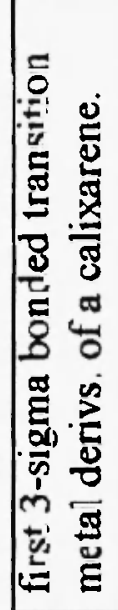 & 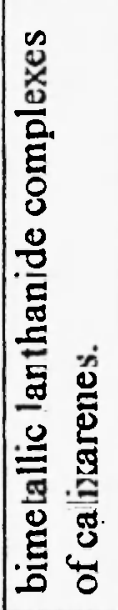 & 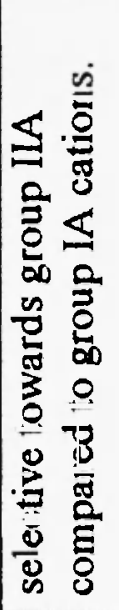 & & 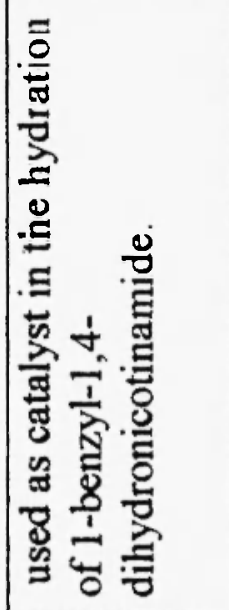 & 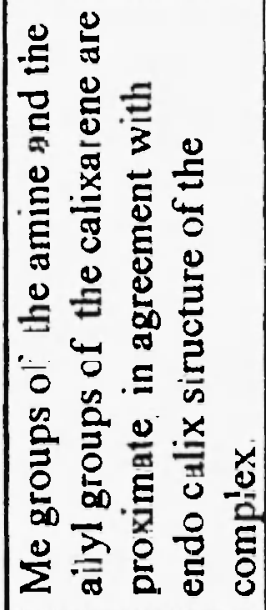 & 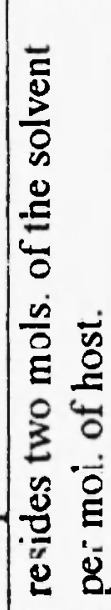 \\
\hline 交 & 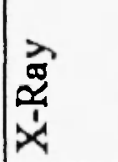 & 苨 & & 1 & 至 & 齐 \\
\hline$E 0$ & 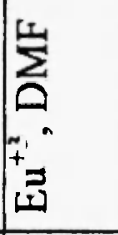 & $\begin{array}{l}5 \\
0 \\
0 \\
0 \\
0\end{array}$ & & 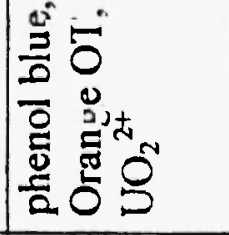 & 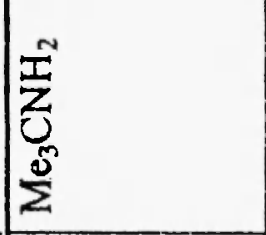 & ठิ \\
\hline 寸 & $\infty$ & $\begin{array}{l}0 \\
0 \\
i \\
i\end{array}$ & & 10 & $\nabla$ & $\infty$ \\
\hline 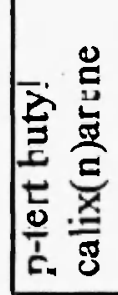 & 衣告 & 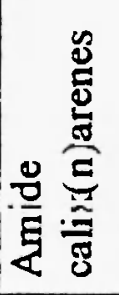 & 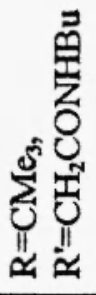 & 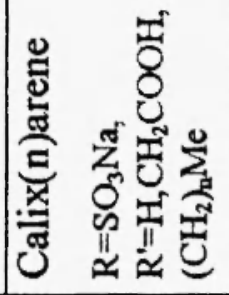 & হ. & 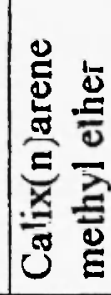 \\
\hline
\end{tabular}




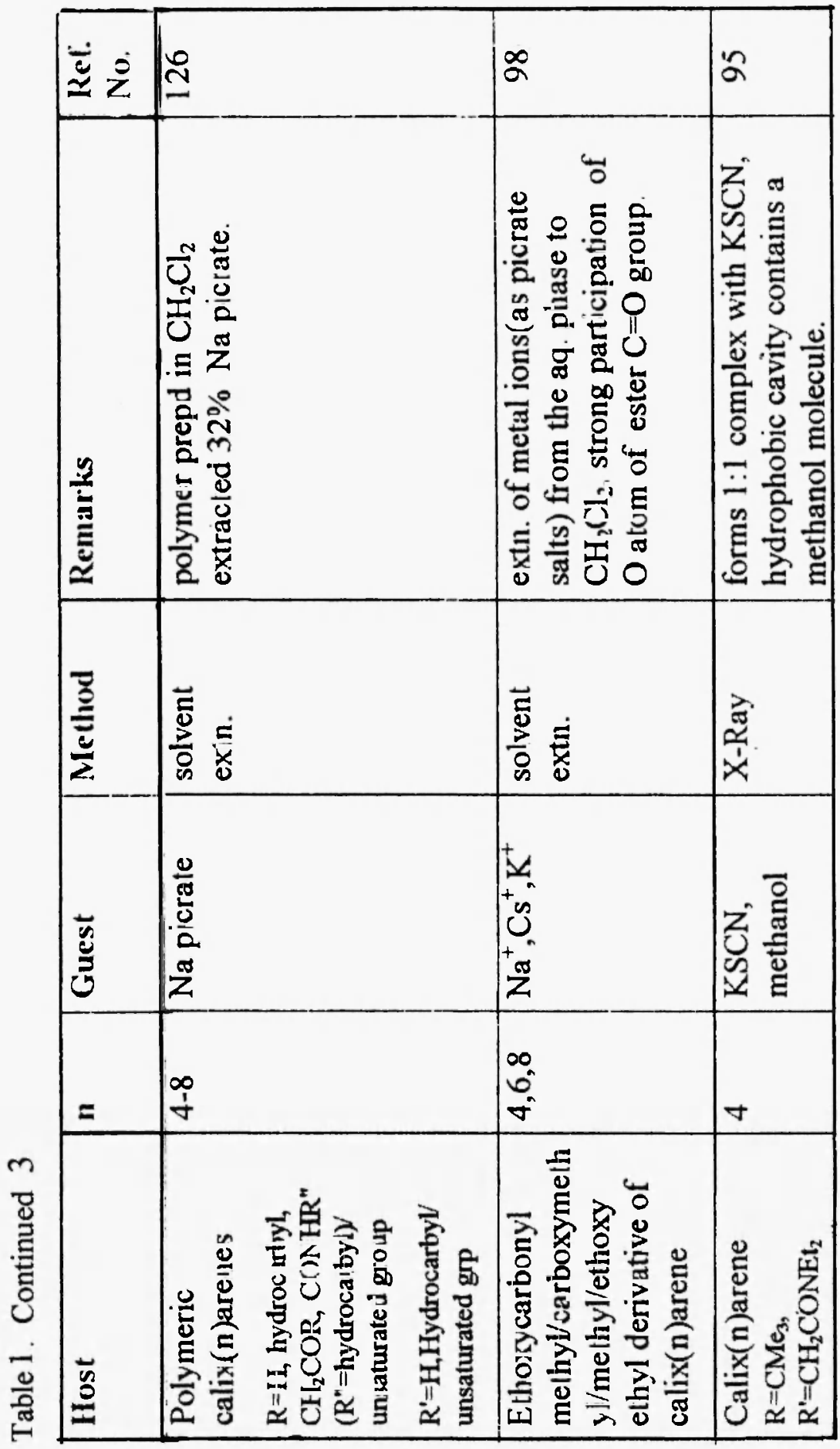




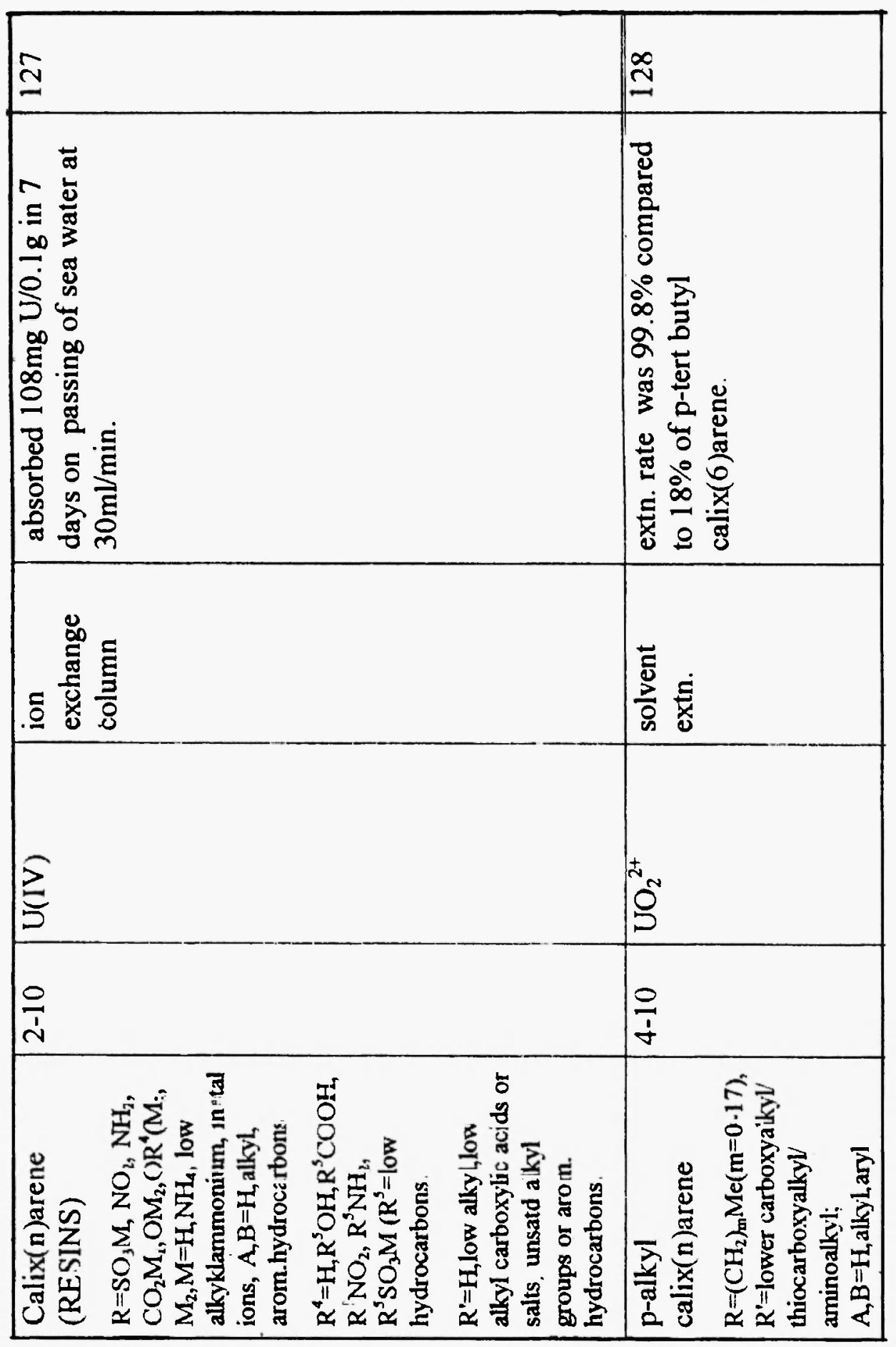




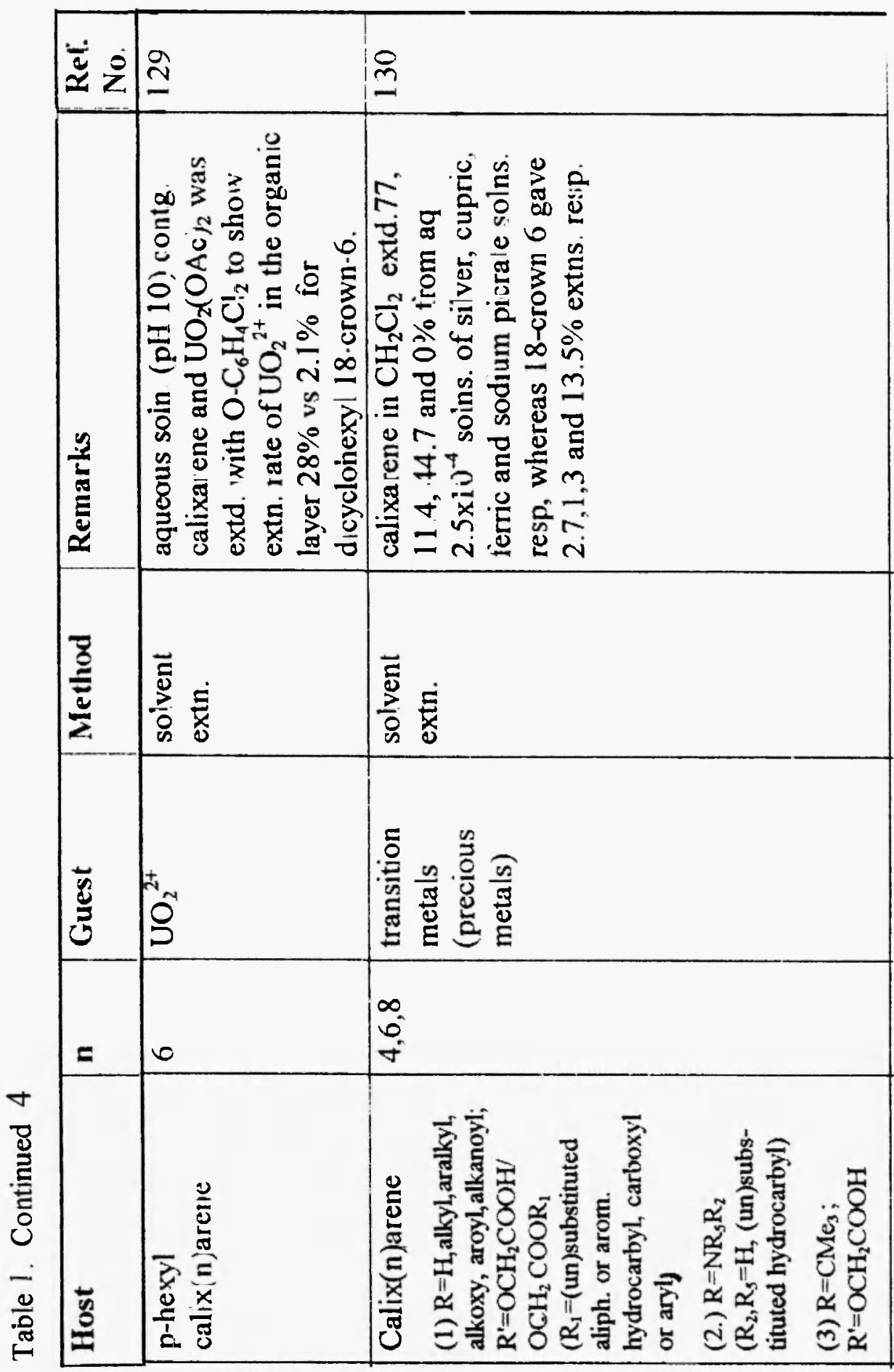




\begin{tabular}{|c|c|c|c|c|c|c|}
\hline $\bar{m}$ & $\stackrel{N}{m}$ & \multicolumn{3}{|l|}{$\ddot{m}$} & $\stackrel{I}{\Phi_{1}}$ & $\ddot{2}$ \\
\hline 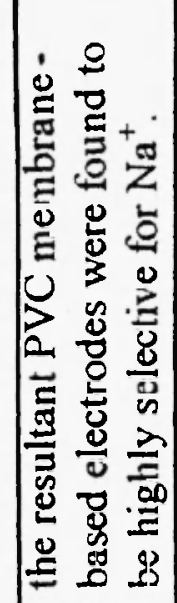 & 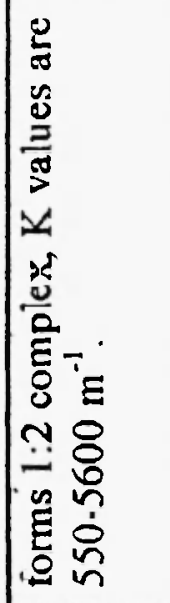 & \multicolumn{3}{|l|}{ 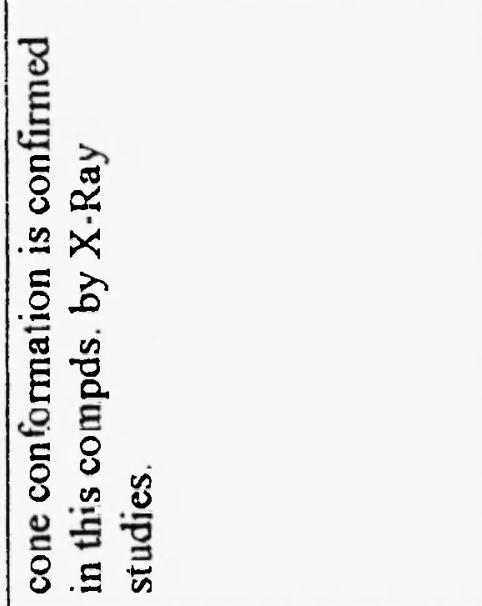 } & 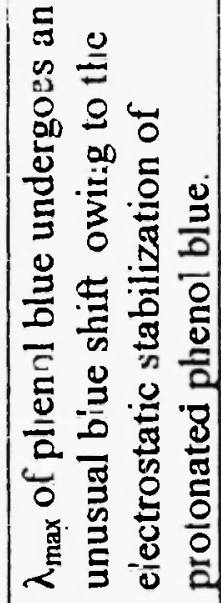 & 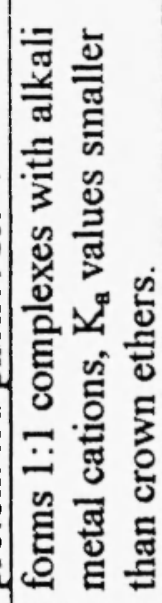 \\
\hline 岕 & $\sum_{\underline{z}}^{\underline{z}}$ & \multicolumn{3}{|l|}{ 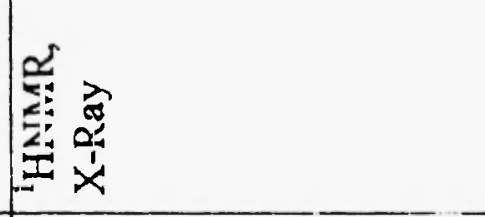 } & 3 & 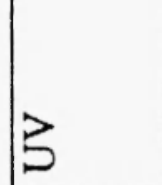 \\
\hline$\left.\right|^{+\infty}$ & 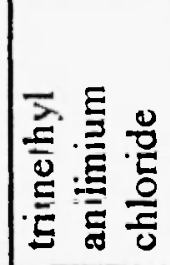 & \multicolumn{3}{|l|}{$t^{+}$} & 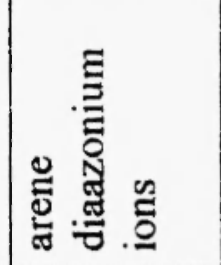 & 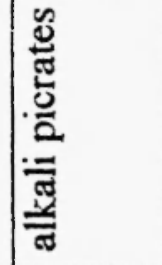 \\
\hline | & $\begin{array}{l}\infty \\
0 \\
-\end{array}$ & \multicolumn{3}{|l|}{$\nabla$} & 10 & $\begin{array}{l}\infty \\
0 \\
\nabla\end{array}$ \\
\hline 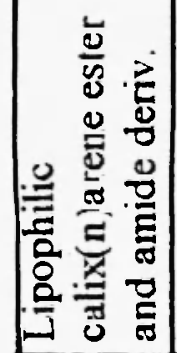 & 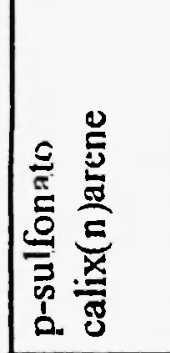 & 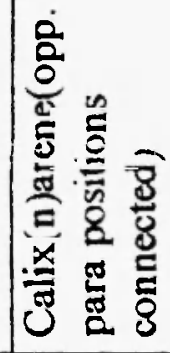 & 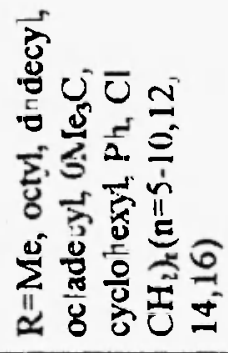 & $\begin{array}{l}T \\
0 \\
\Pi 1 \\
0\end{array}$ & 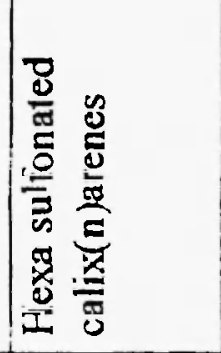 & 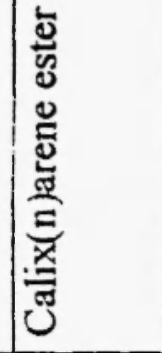 \\
\hline
\end{tabular}




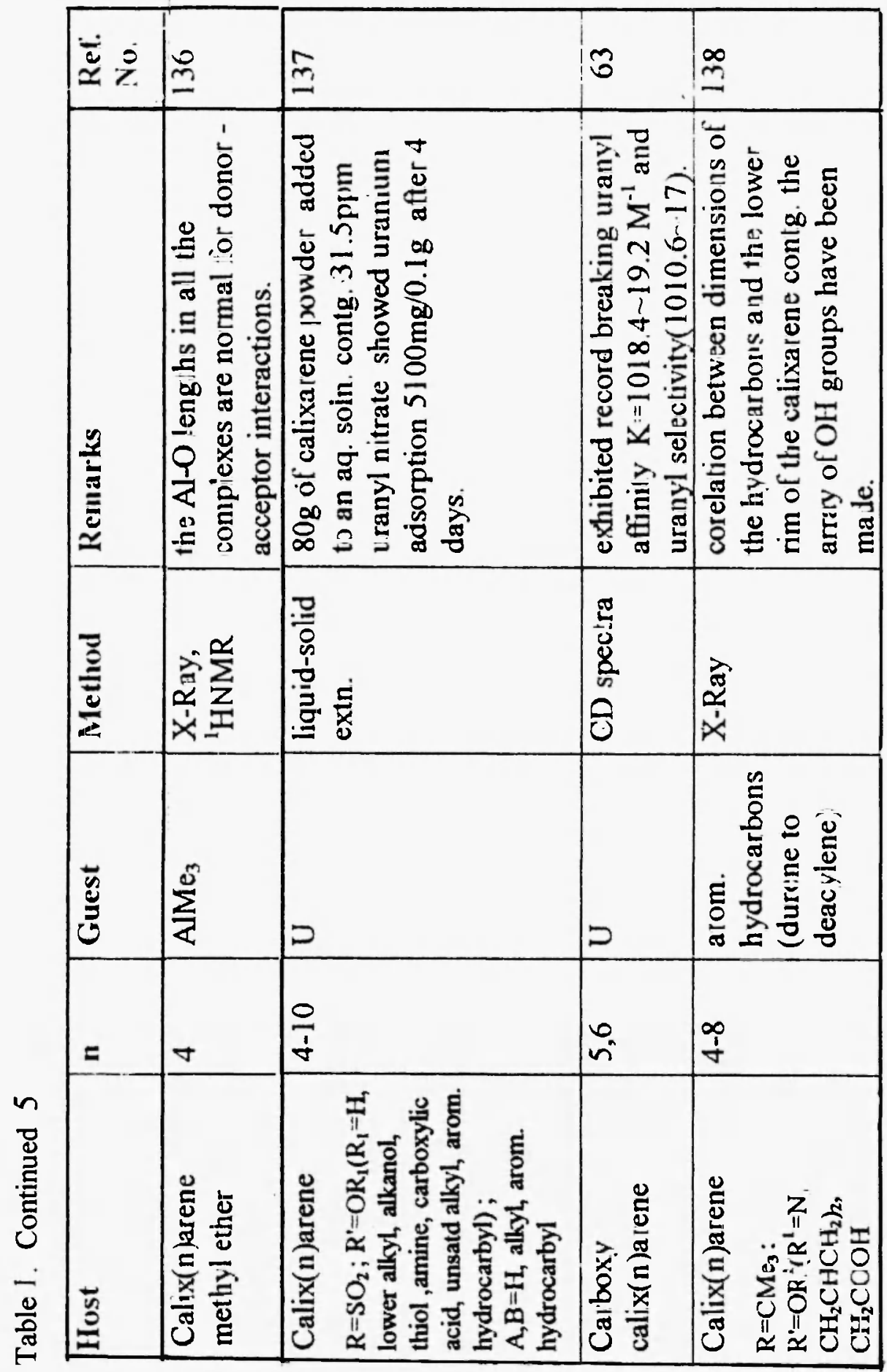




\begin{tabular}{|c|c|c|c|}
\hline$\stackrel{g}{-}$ & I엉 & $\Xi$ & 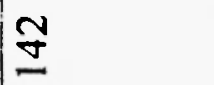 \\
\hline 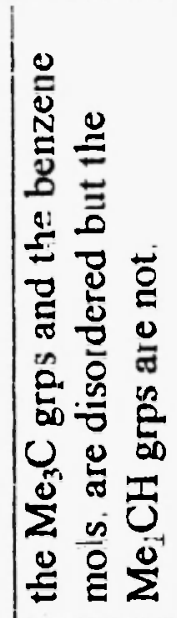 & 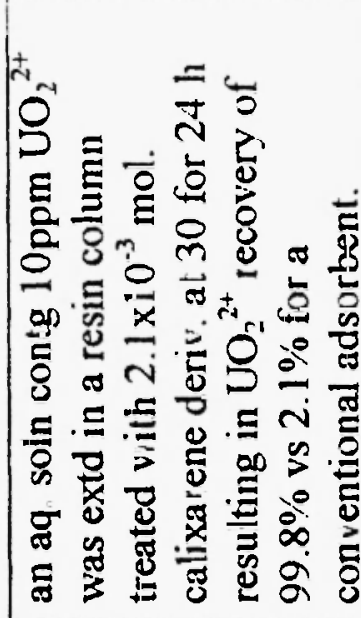 & 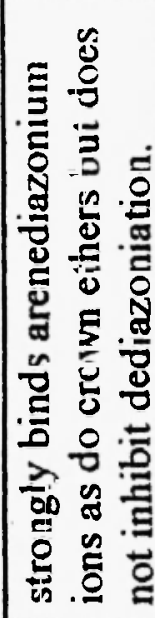 & 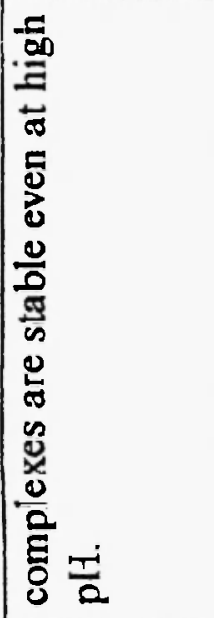 \\
\hline 突 & 施 & 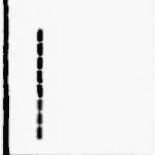 & 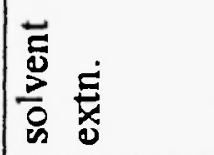 \\
\hline 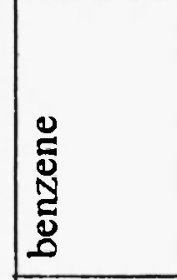 & $\overbrace{}^{4}$ & 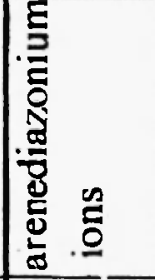 & 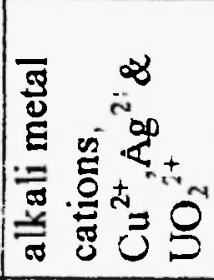 \\
\hline 0 & 0 & 10 & $\begin{array}{l}\infty \\
0\end{array}$ \\
\hline 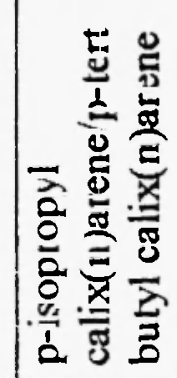 & 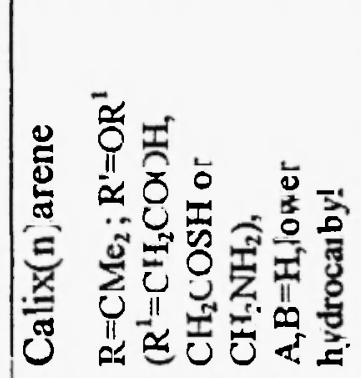 & 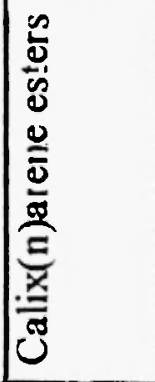 & 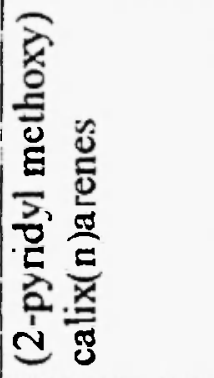 \\
\hline
\end{tabular}




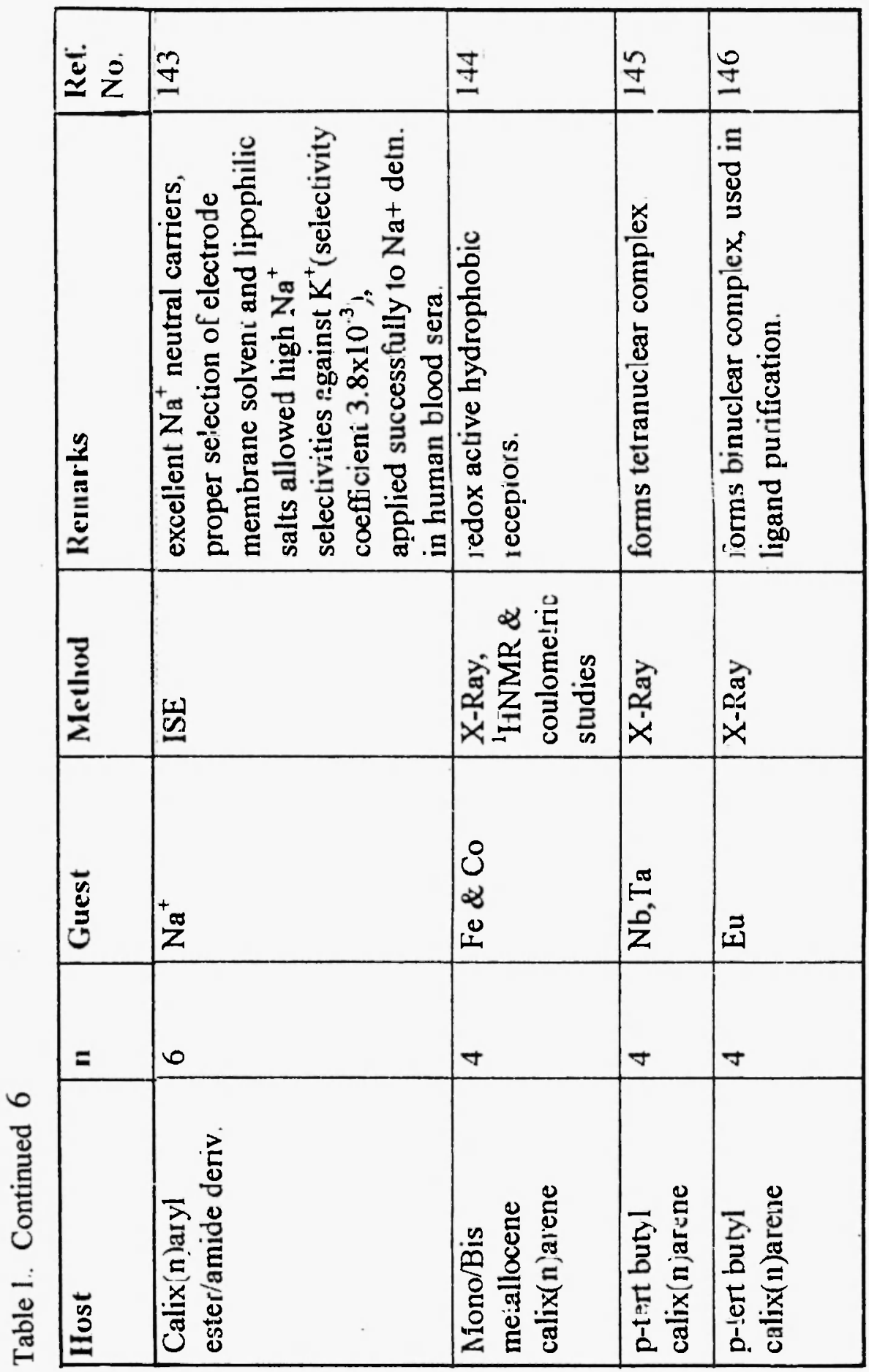




\begin{tabular}{|c|c|c|}
\hline I & $\mid \begin{array}{l}\infty \\
\pm\end{array}$ & 昌 \\
\hline 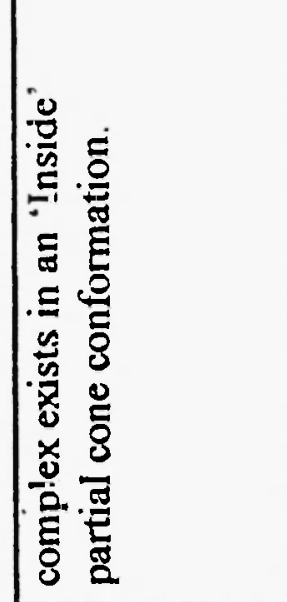 & 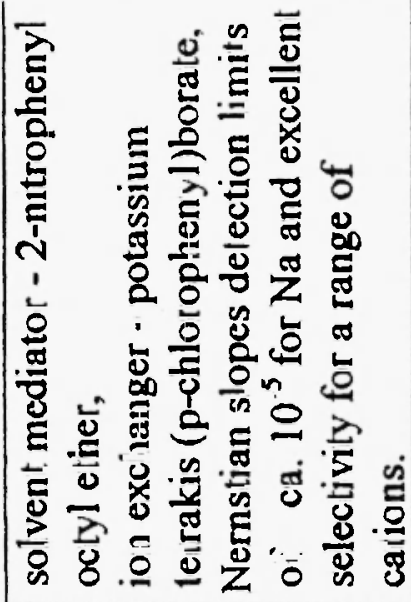 & 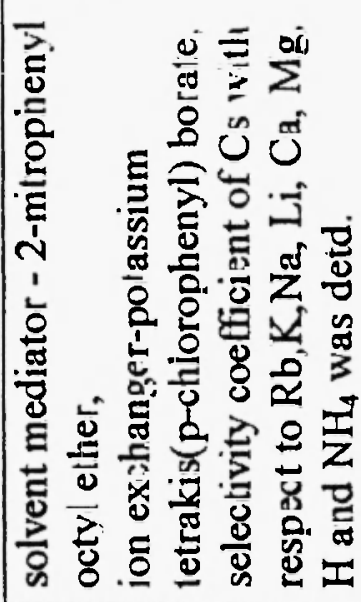 \\
\hline 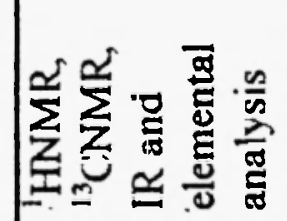 & 峴 & 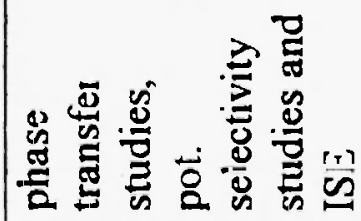 \\
\hline 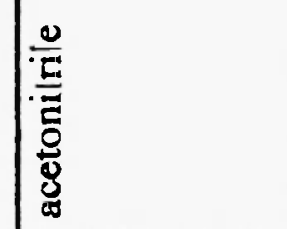 & ${ }^{+}$ & $\begin{array}{l}\frac{0}{21} \\
\infty \\
0\end{array}$ \\
\hline$\nabla$ & $\nabla$ & 10 \\
\hline 农 & 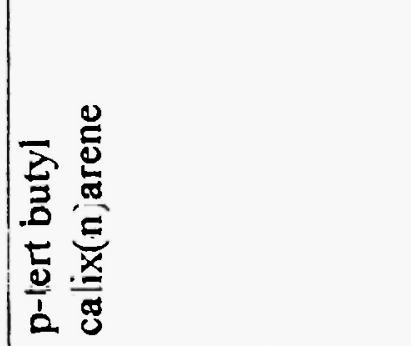 & 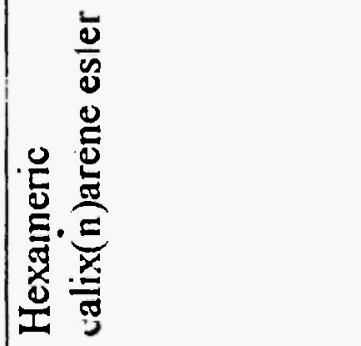 \\
\hline
\end{tabular}




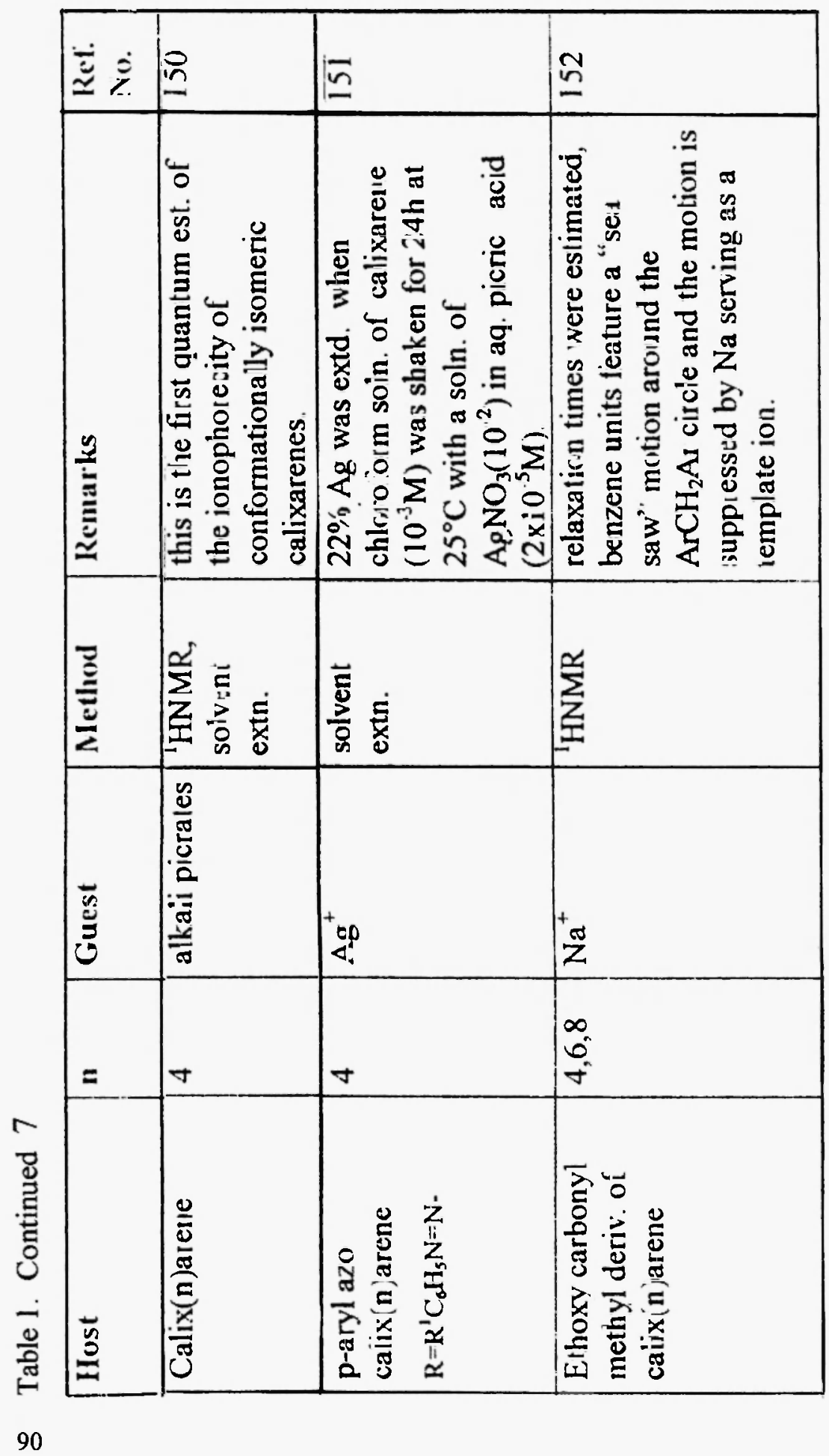




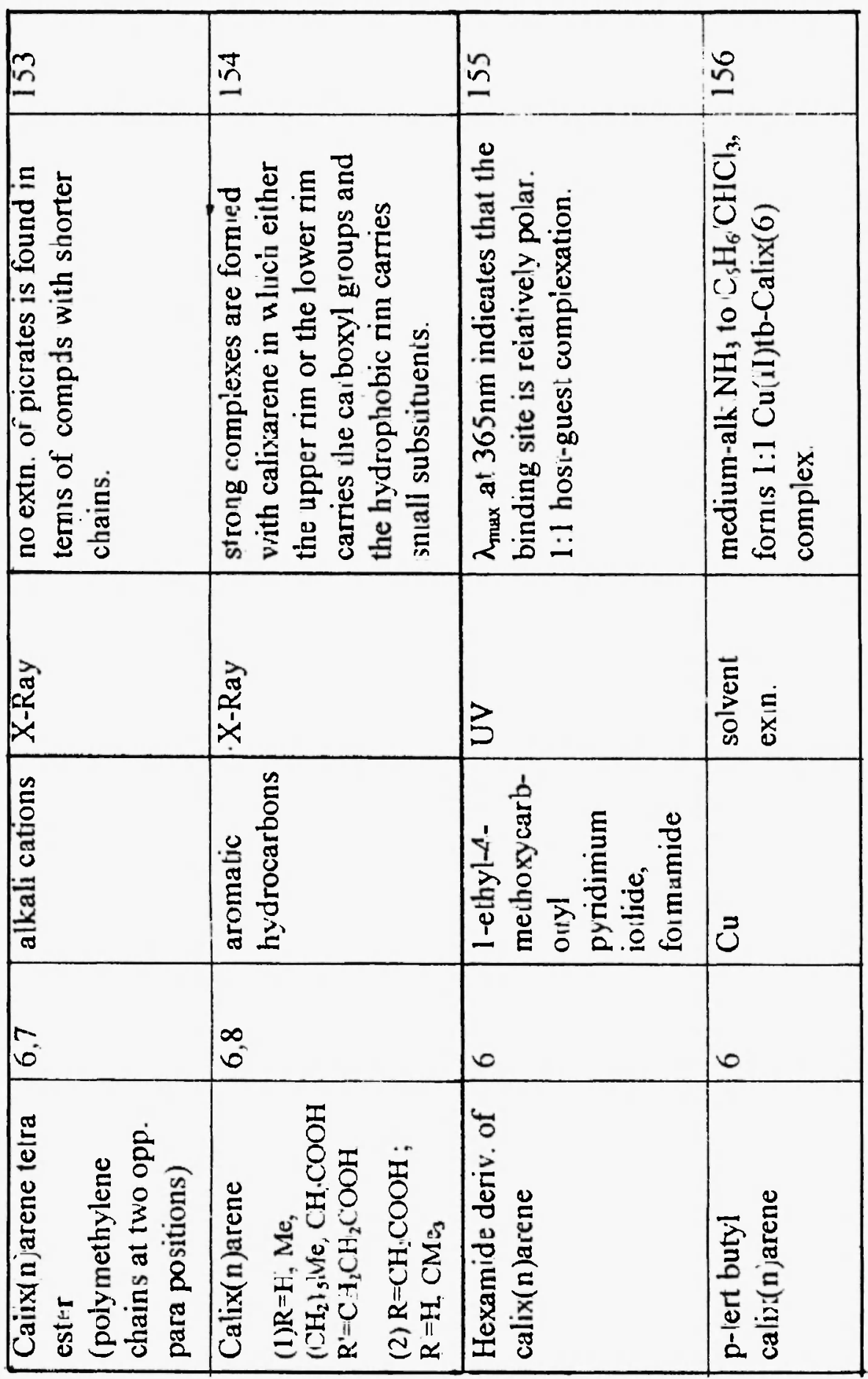




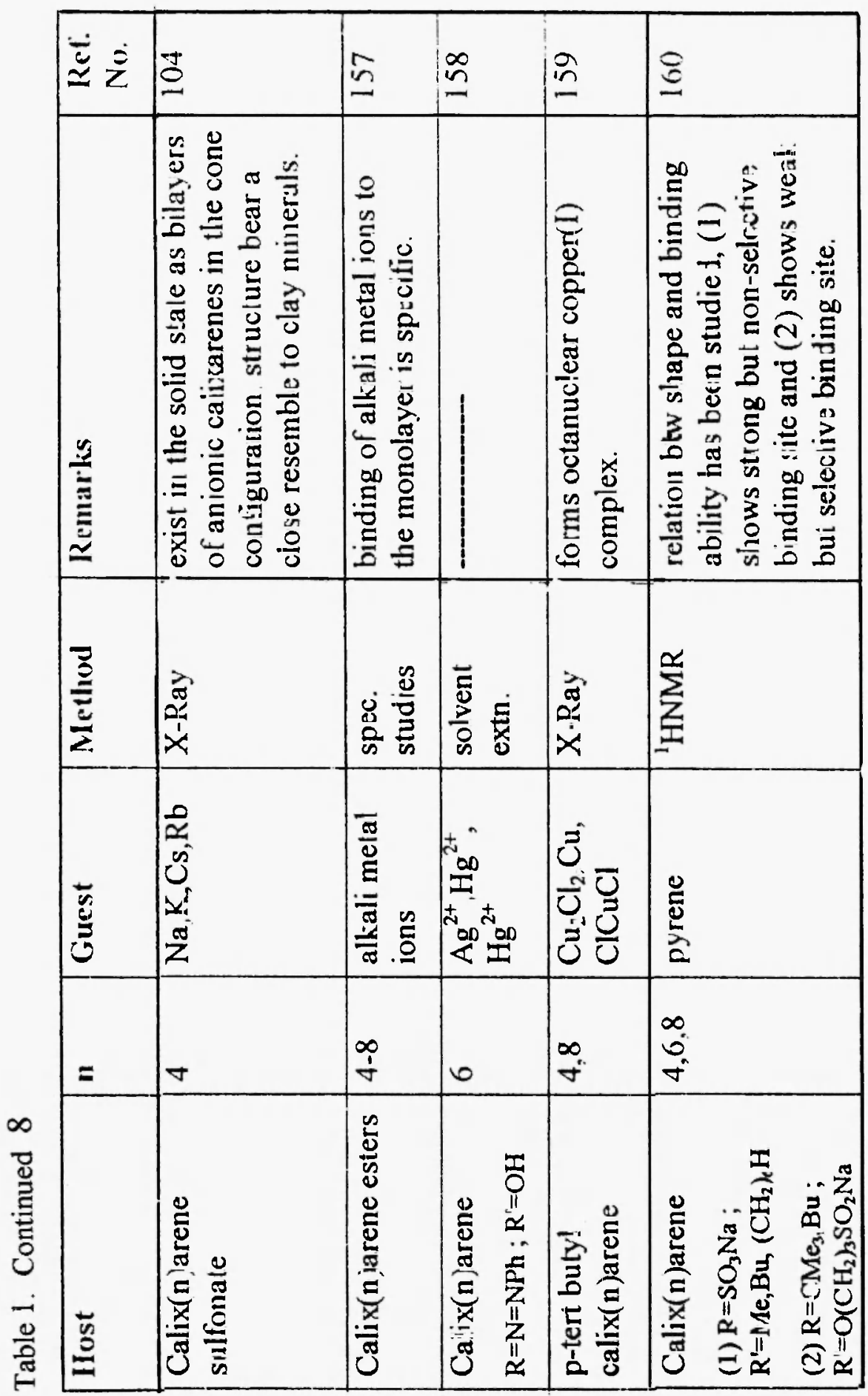




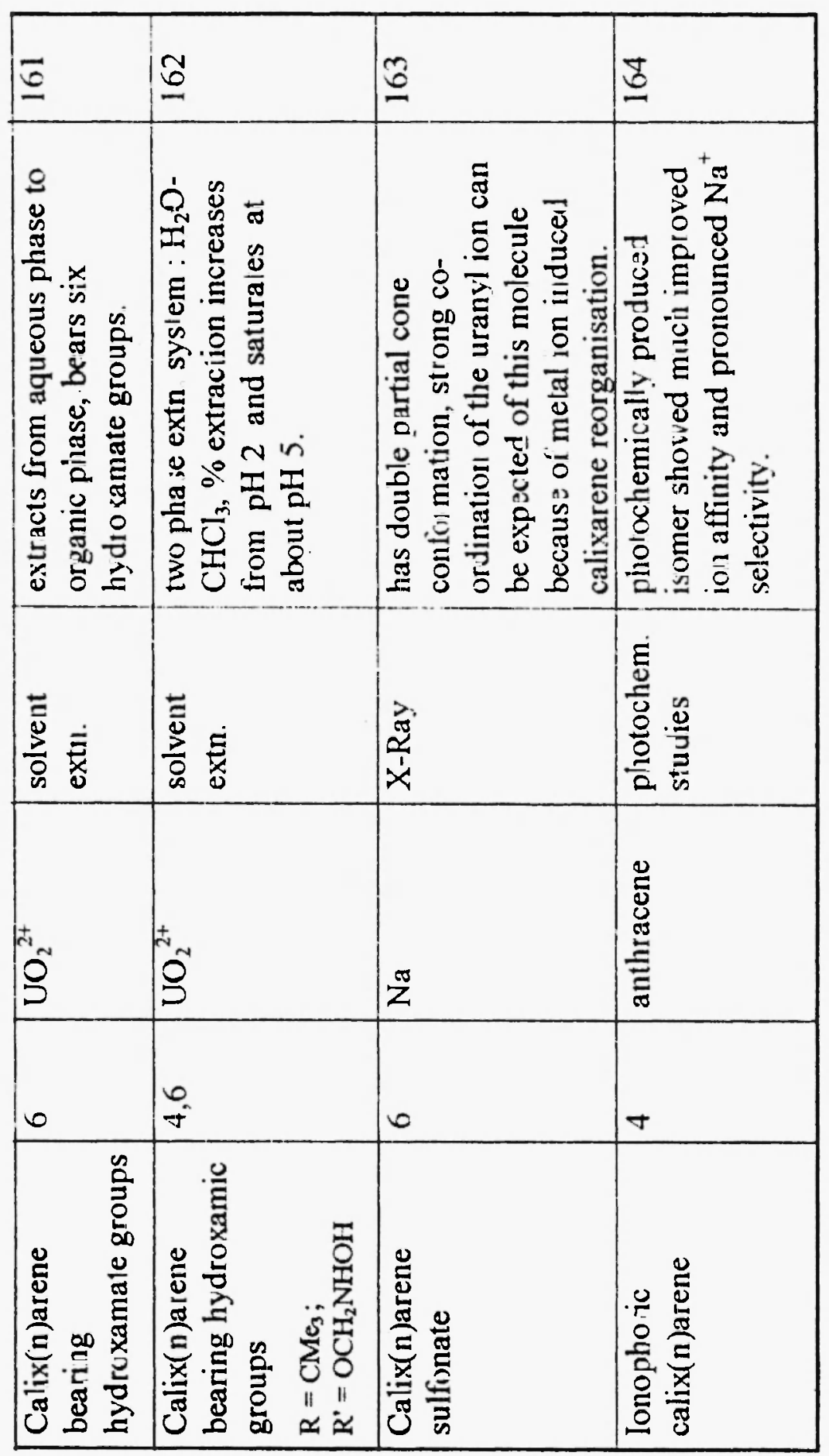




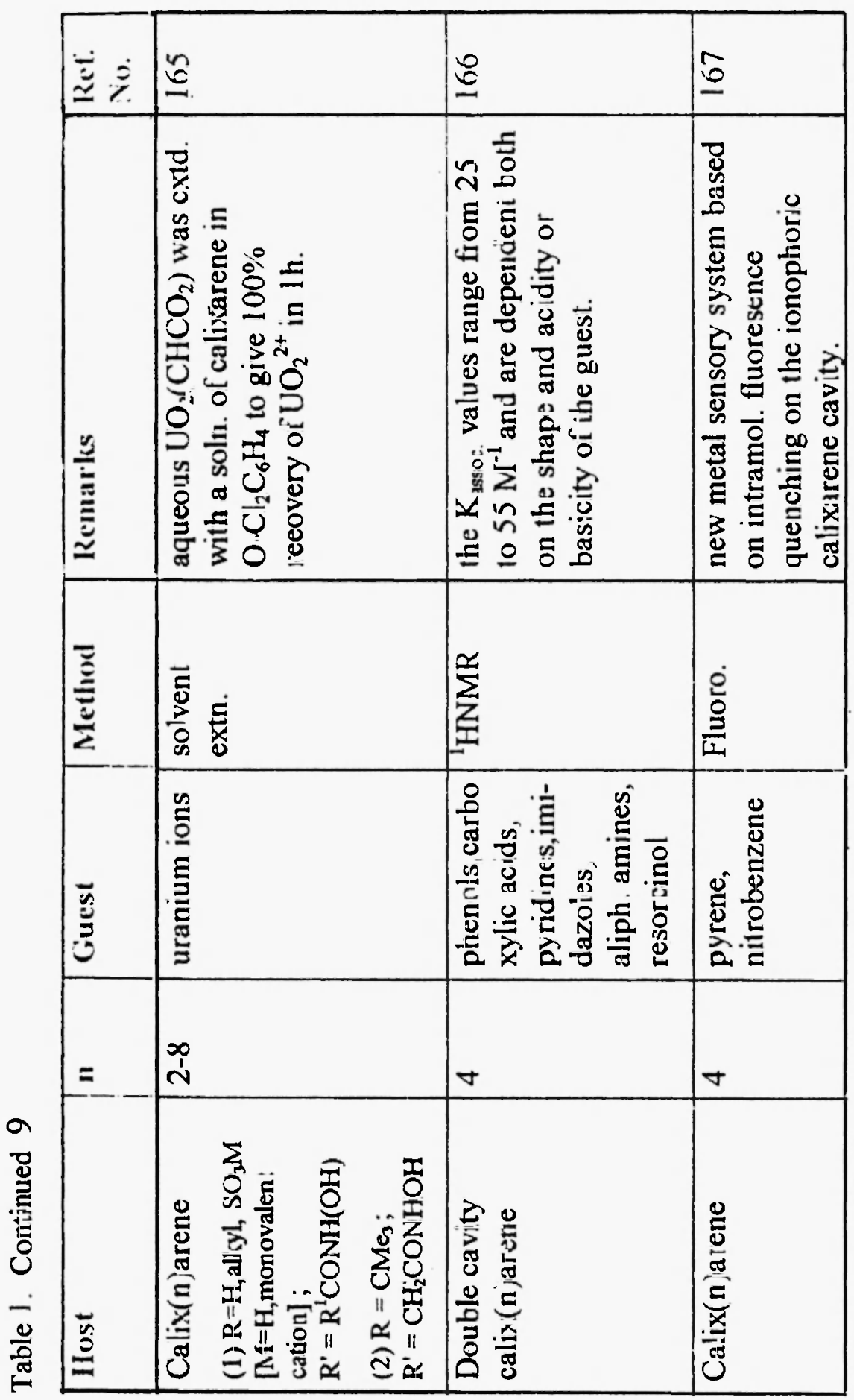




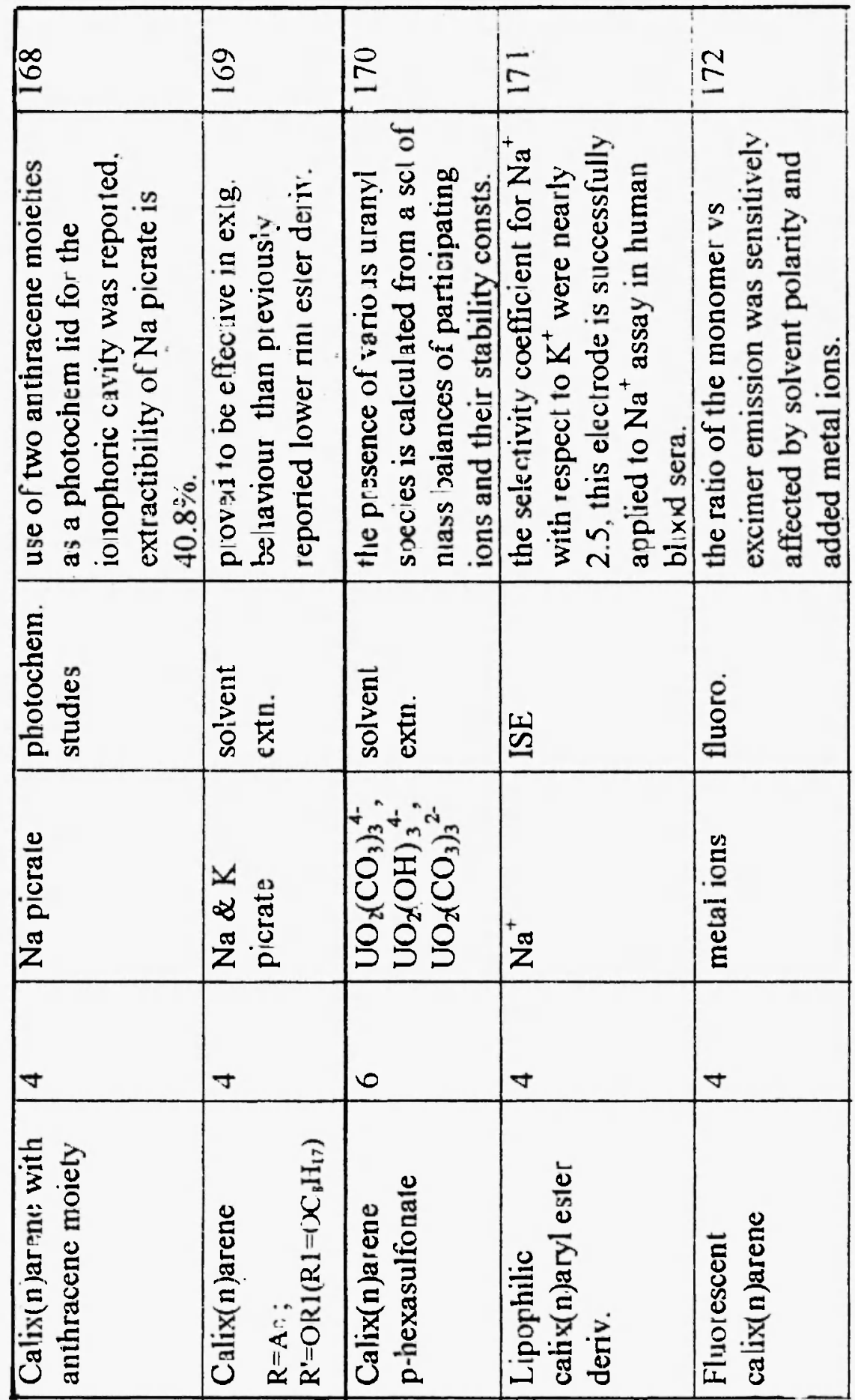




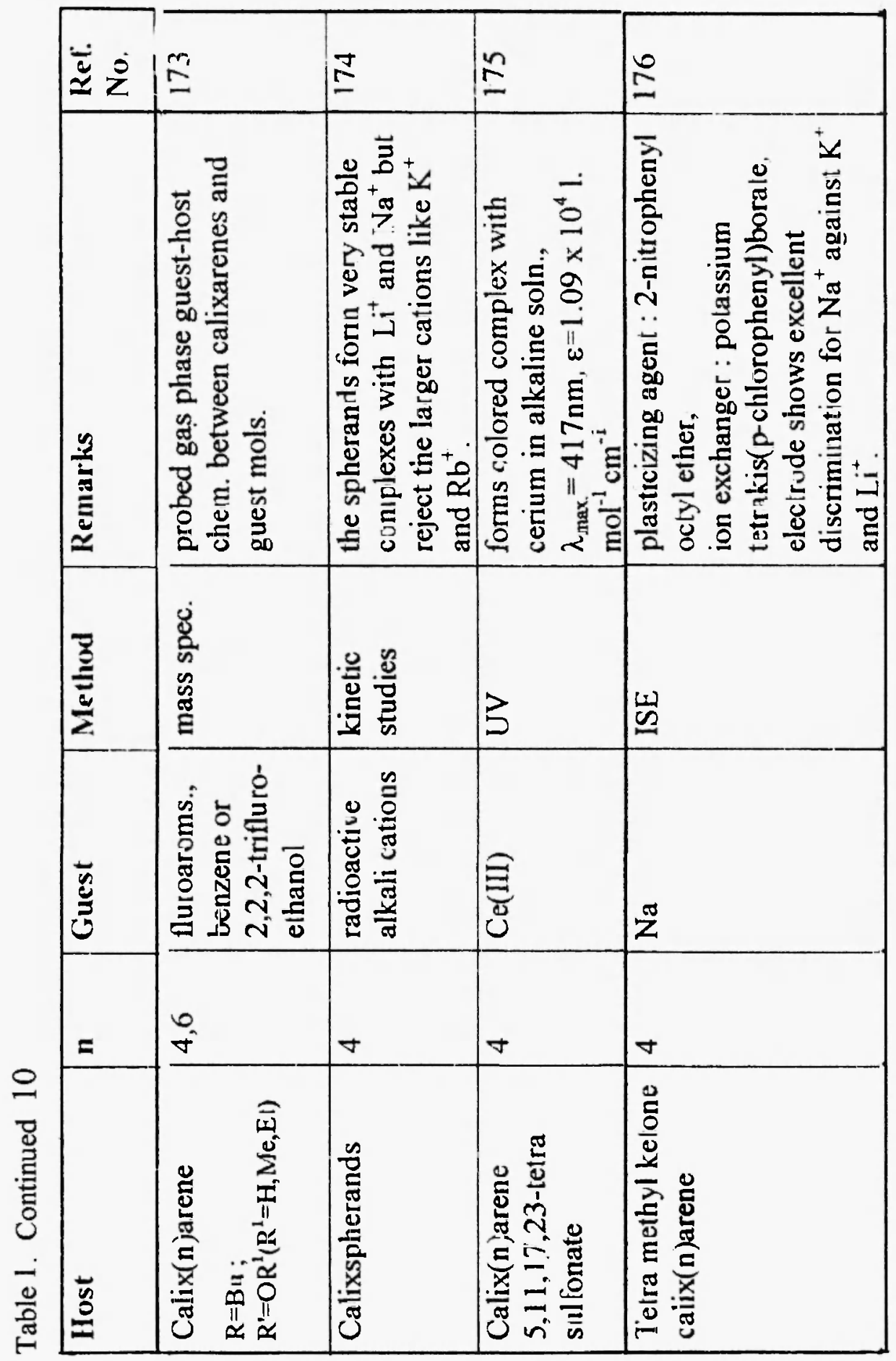




\begin{tabular}{|c|c|c|c|c|c|}
\hline$\Sigma$ & 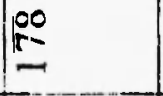 & 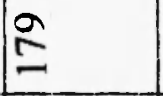 & 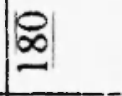 & $\infty$ & $\infty$ \\
\hline I & 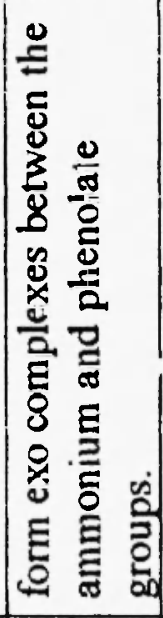 & 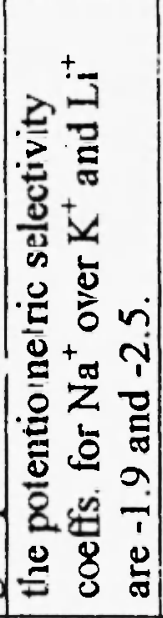 & 1 & 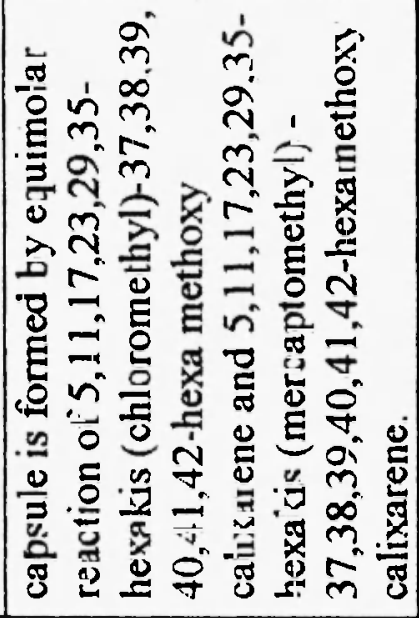 & 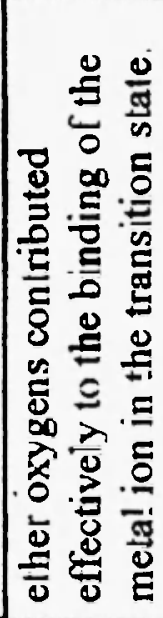 \\
\hline 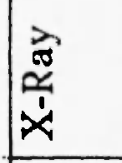 & 兽 & 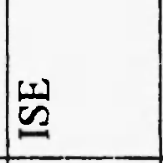 & 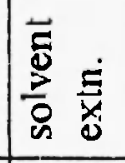 & 1 & 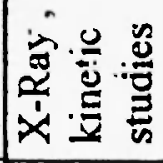 \\
\hline $\begin{array}{l}\vec{U} \\
\dot{0} \\
\dot{0}\end{array}$ & 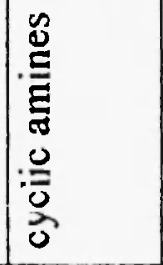 & $I^{+}$ & \pm- & 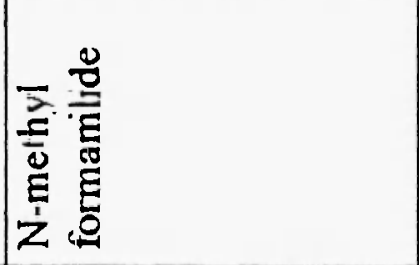 & 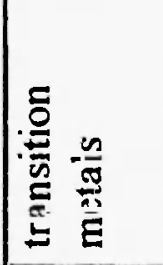 \\
\hline$\nabla$ & 6 & $\nabla$ & 7 & $\nabla$ & $\nabla$ \\
\hline 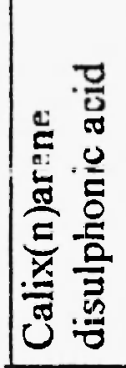 & 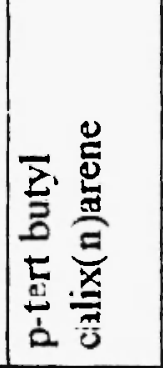 & 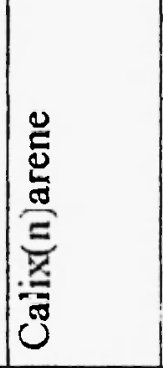 & 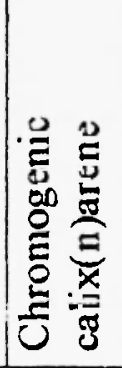 & 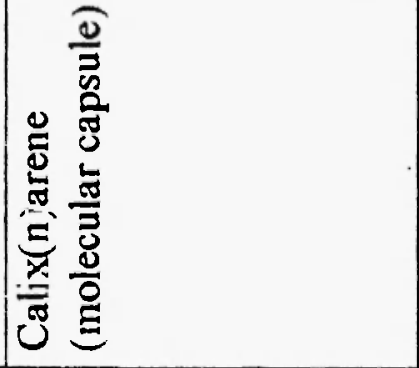 & 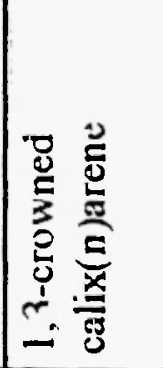 \\
\hline
\end{tabular}




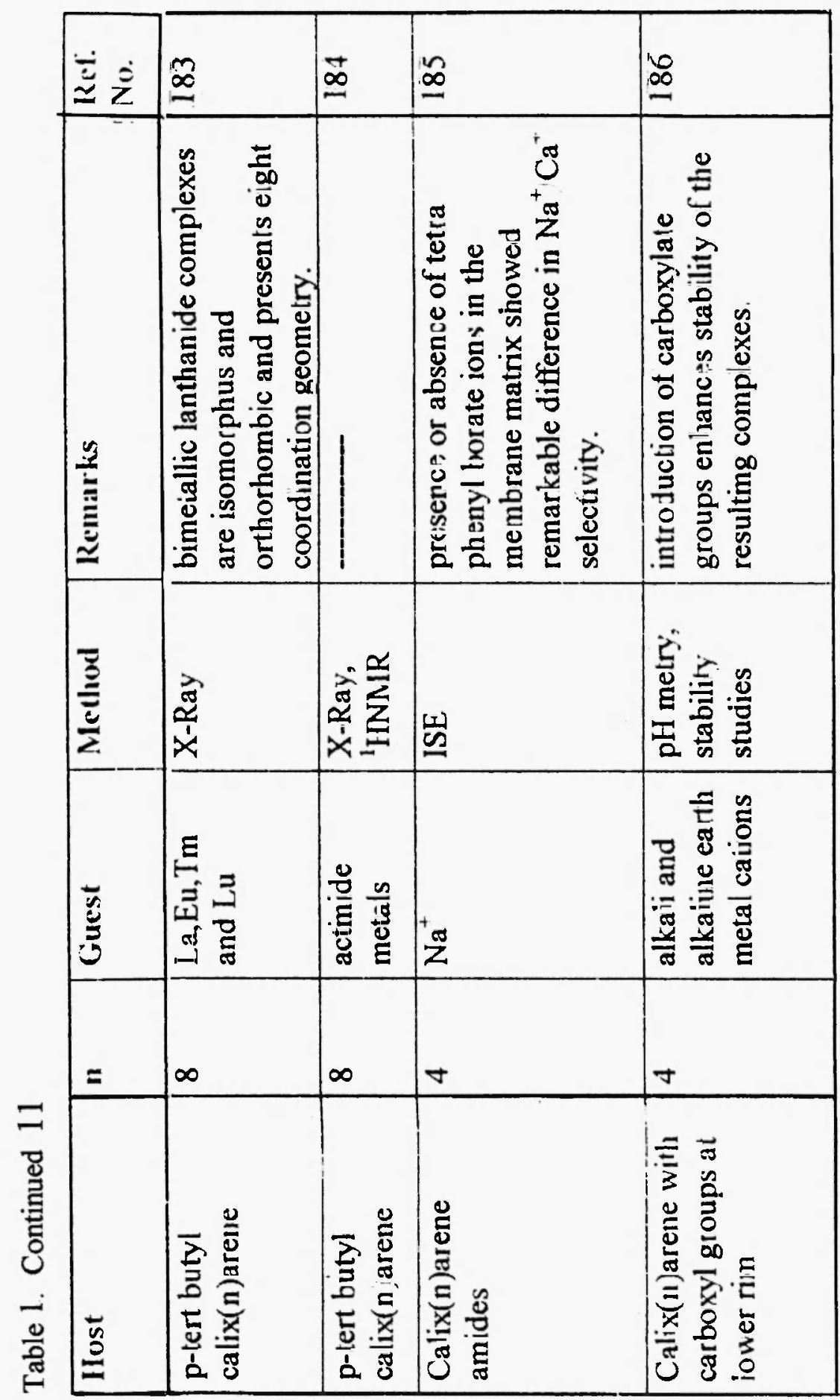




\begin{tabular}{|c|c|c|c|c|}
\hline$\infty$ & $\begin{array}{l}\infty \\
\infty\end{array}$ & $\infty$ & 으 & $a$ \\
\hline 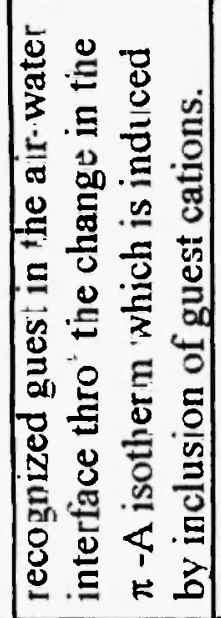 & 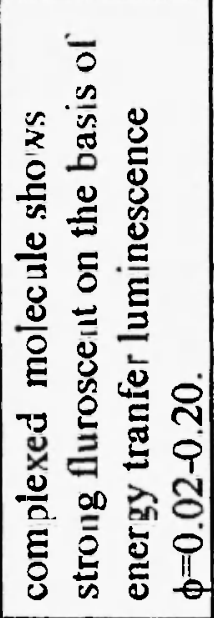 & 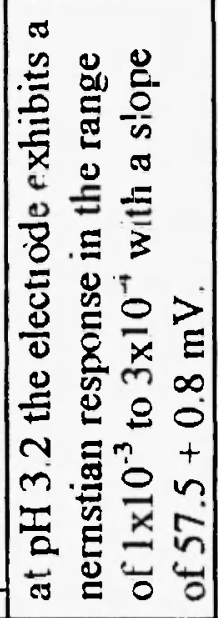 & 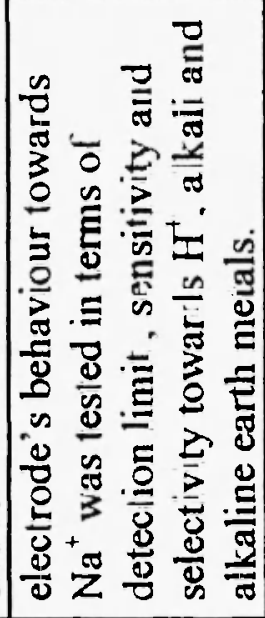 & 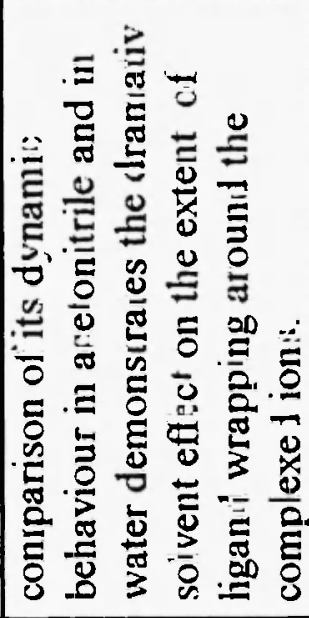 \\
\hline i & 鸹总 & $\stackrel{2}{2}$ & $\sqrt{2}$ & 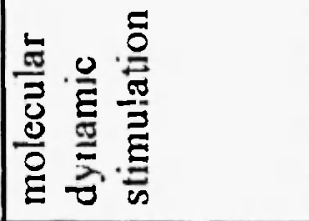 \\
\hline ㄹ. & مث & 忌. & ${ }^{+}$ & 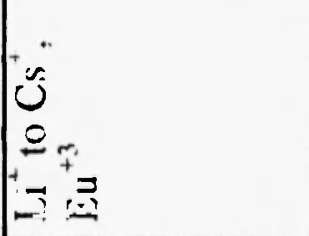 \\
\hline$\nabla$ & $\sigma$ & 0 & $\nabla$ & ナ \\
\hline$\frac{\frac{0}{0}}{\frac{0}{0}}$ & 兽 & 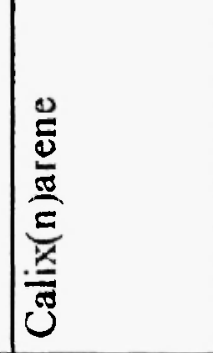 & 造 & 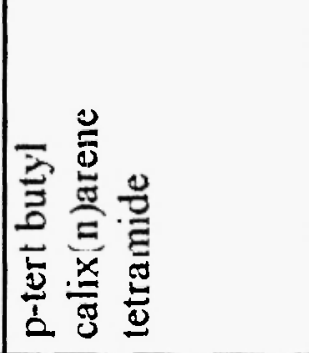 \\
\hline
\end{tabular}




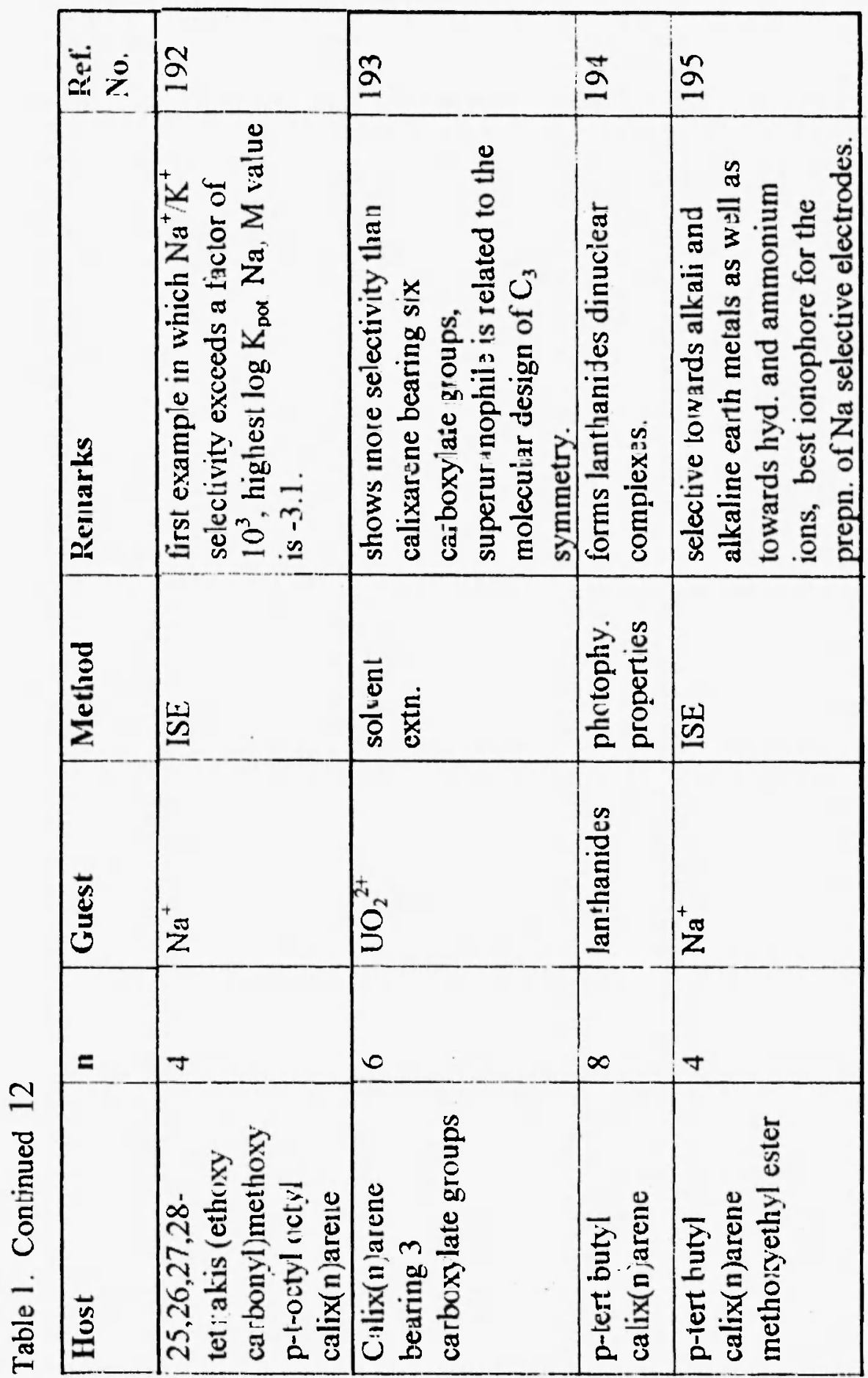




\begin{tabular}{|c|c|c|c|}
\hline$\stackrel{\circ}{\circ}$ & I & $\stackrel{\infty}{\varrho}$ & 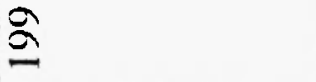 \\
\hline 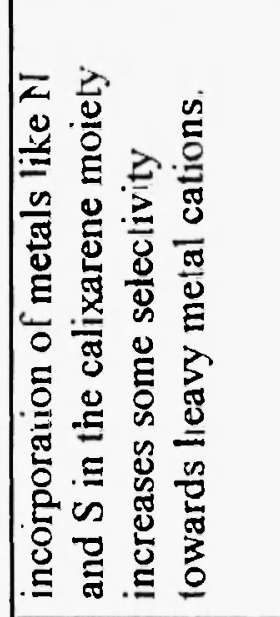 & 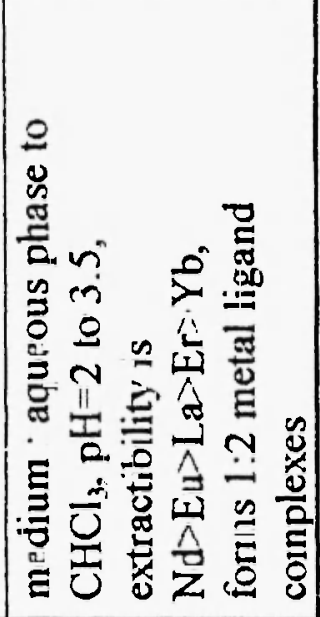 & 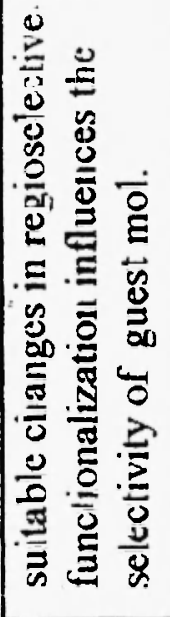 & 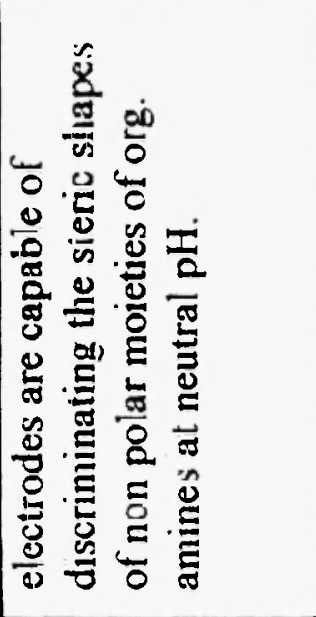 \\
\hline$\stackrel{0}{2}$ & 离 & 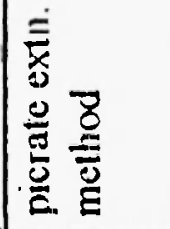 & 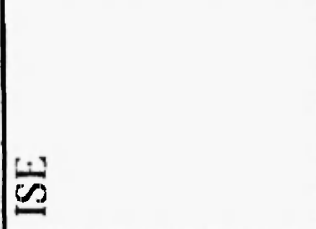 \\
\hline 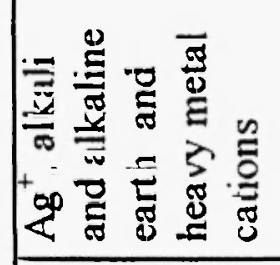 & 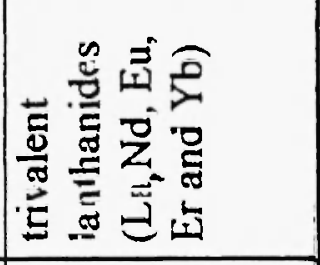 & $+\frac{\pi}{2}$ & 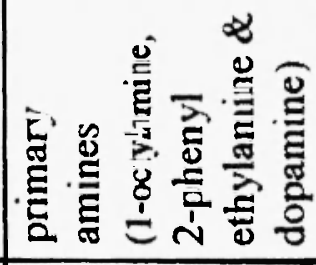 \\
\hline$\checkmark$ & 0 & $\nabla$ & 6 \\
\hline 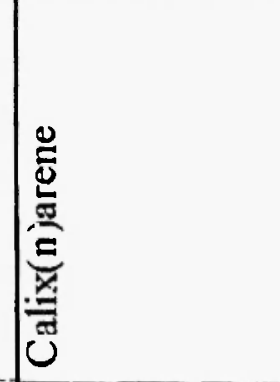 & 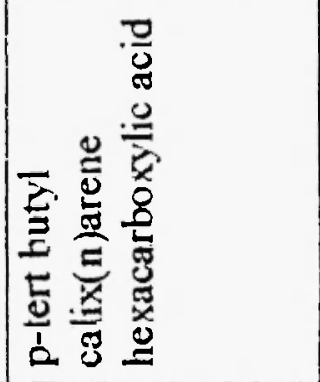 & 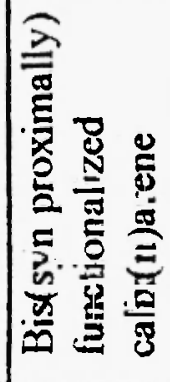 & 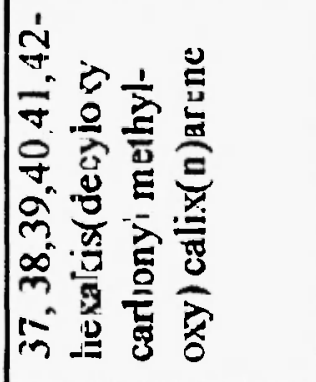 \\
\hline
\end{tabular}




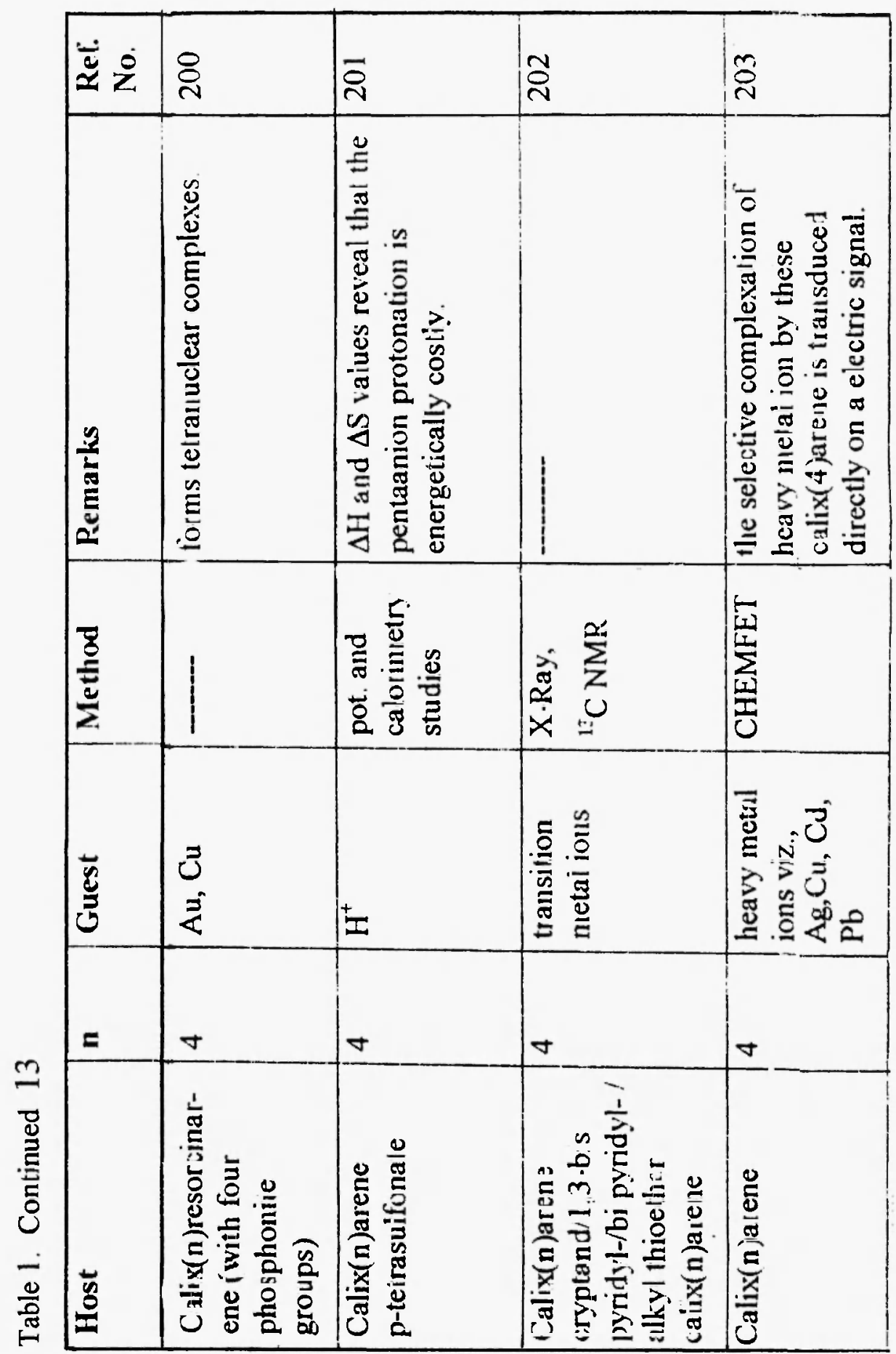




\begin{tabular}{|c|c|c|c|c|}
\hline Jे & $\stackrel{\sim}{\curvearrowright}$ & 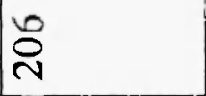 & 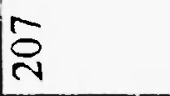 & 疋 \\
\hline 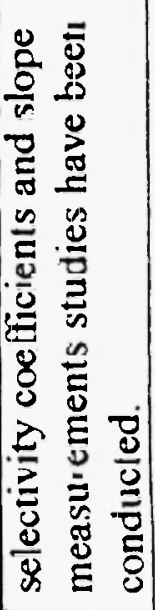 & 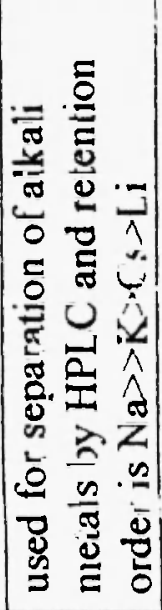 & 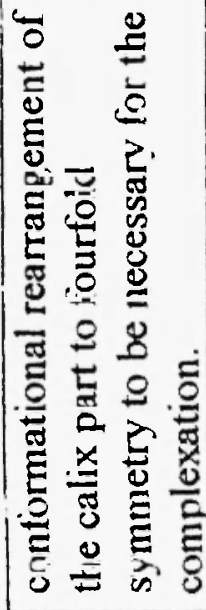 & 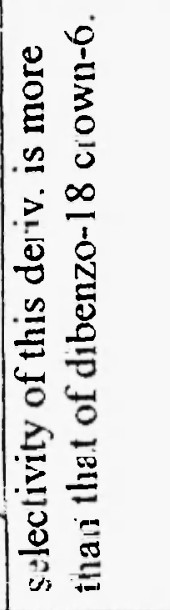 & 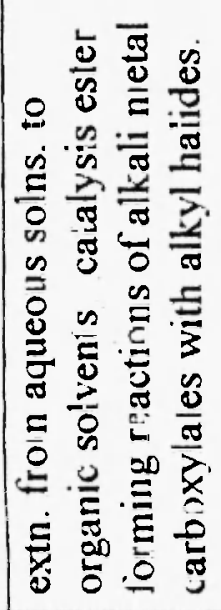 \\
\hline$\ddot{\omega}$ & 足 & 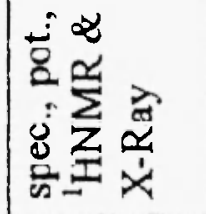 & 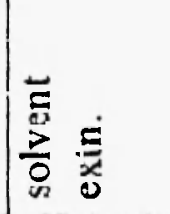 & 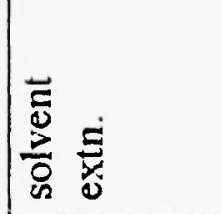 \\
\hline in & $\begin{array}{l}3 \\
3 \\
0 \\
0 \\
z \\
z\end{array}$ & $\mid \begin{array}{l}20 \\
\vdots \\
z \\
z \\
z\end{array}$ & 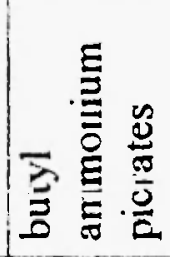 & 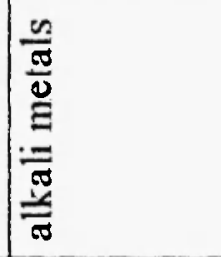 \\
\hline $\begin{array}{l}\infty \\
1 \\
m \\
m\end{array}$ & $\nabla$ & J & 10 & 10 \\
\hline 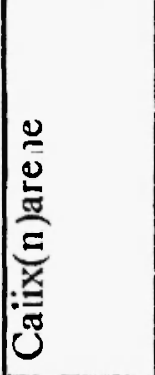 & 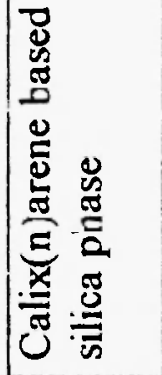 & 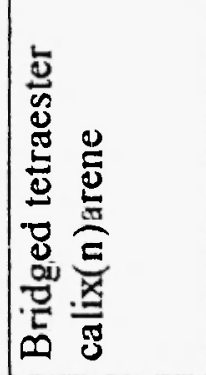 & 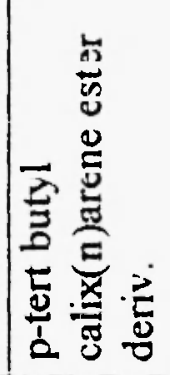 & 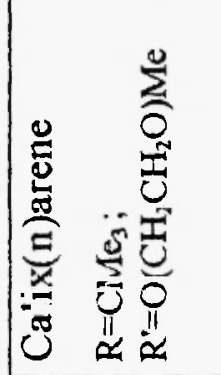 \\
\hline
\end{tabular}




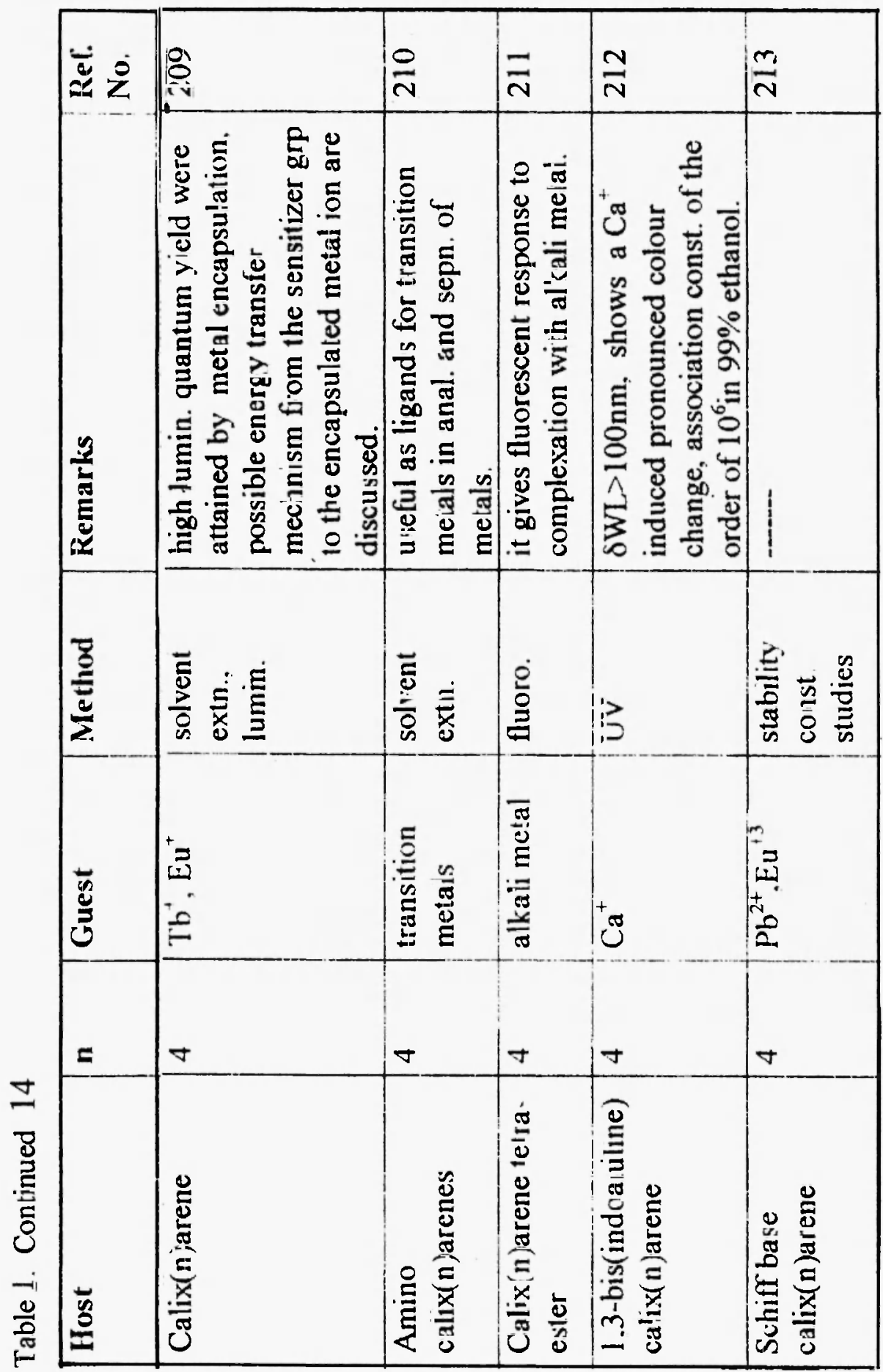




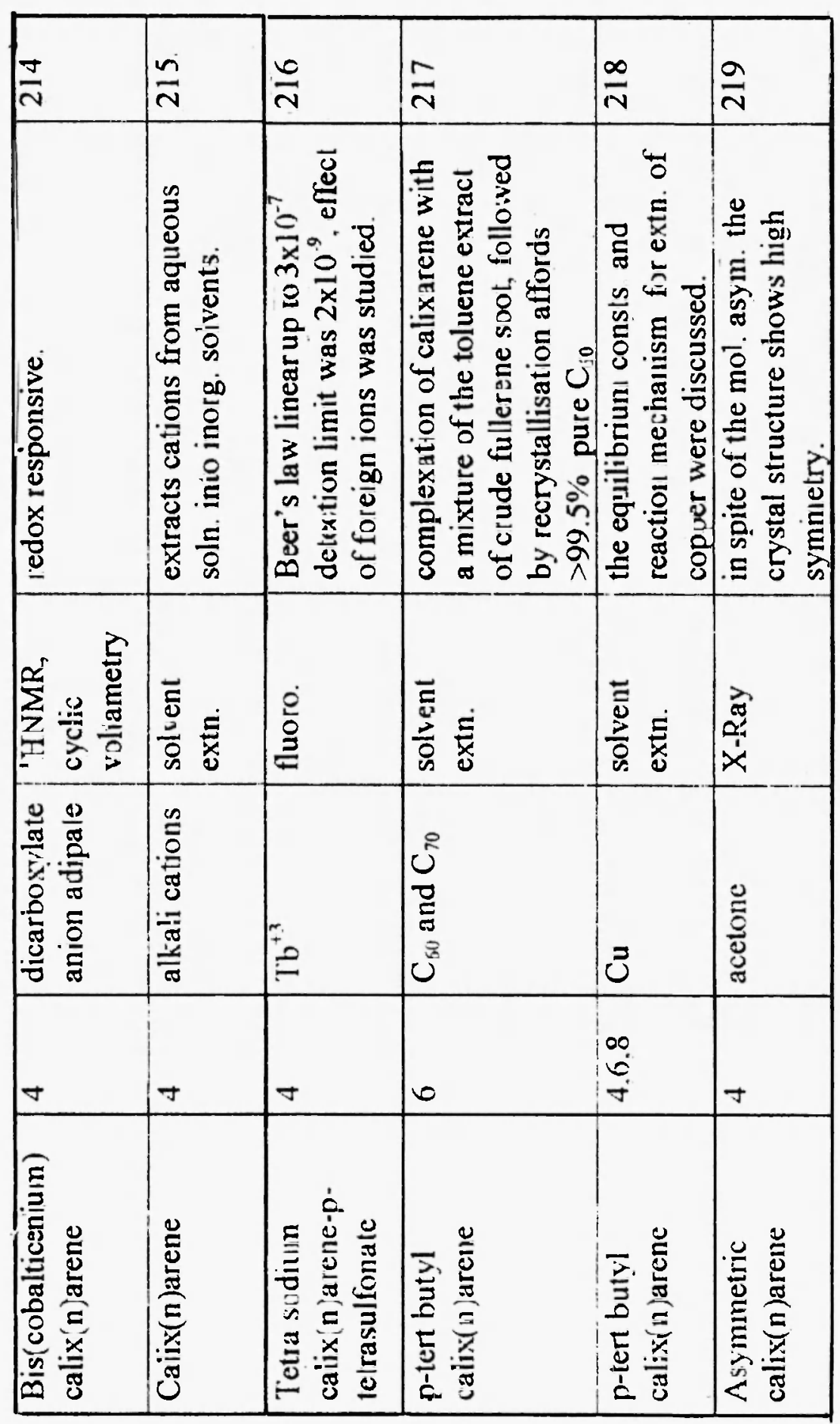




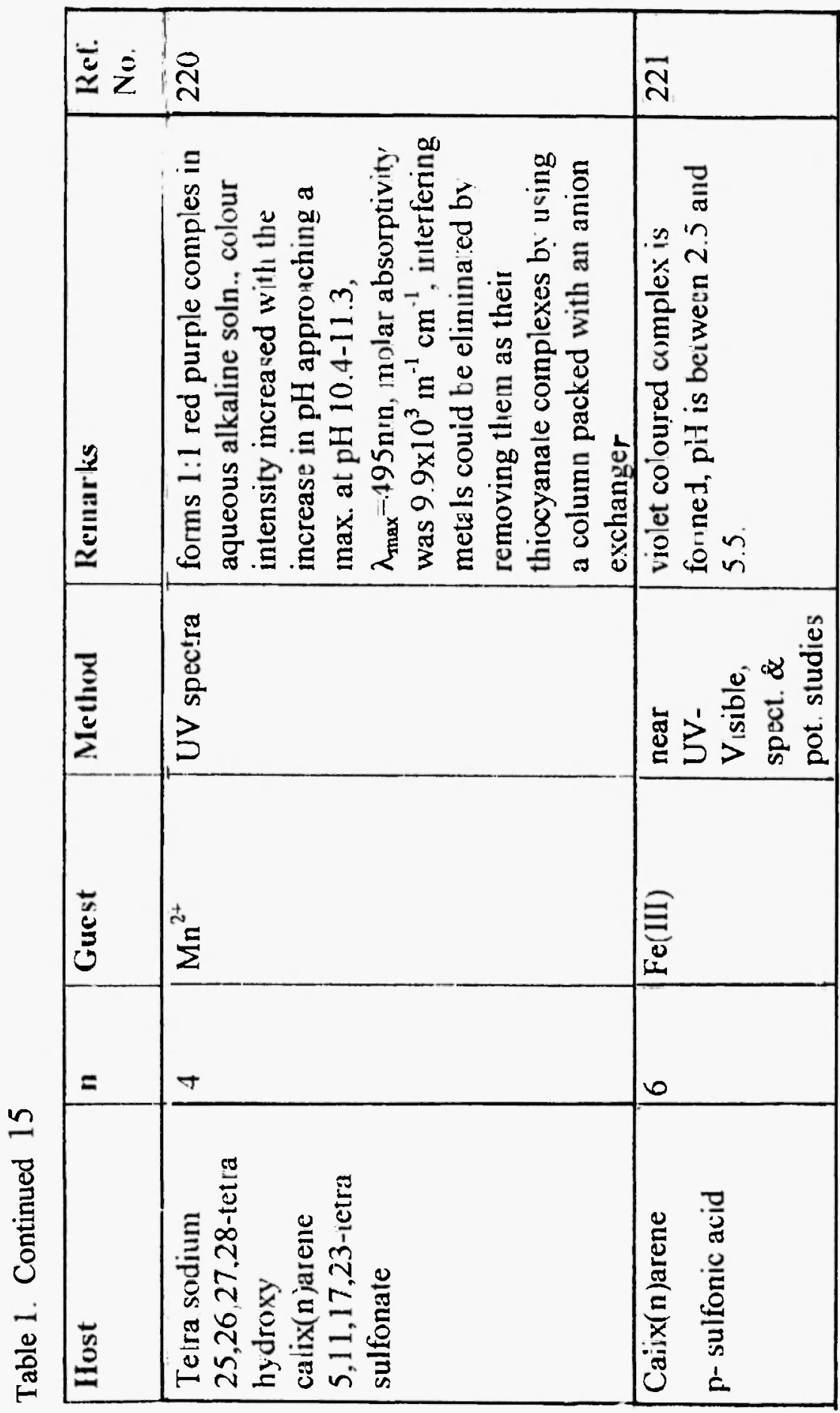




\begin{tabular}{|c|c|c|c|c|}
\hline$\underset{N}{\mathbb{N}}$ & $\stackrel{N}{N}$ & İ & $\stackrel{\sim}{N}$ & $\stackrel{\sim}{\sim}$ \\
\hline 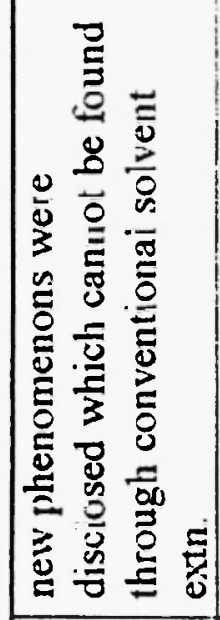 & 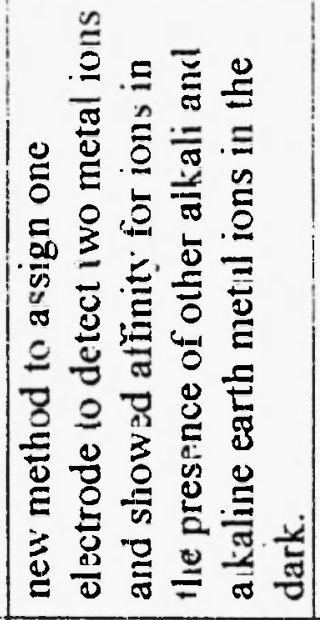 & 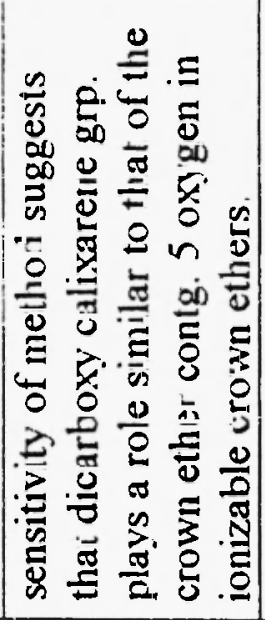 & 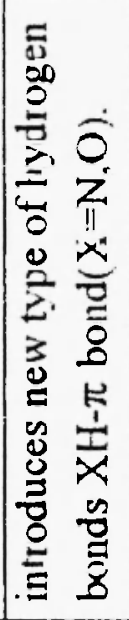 & 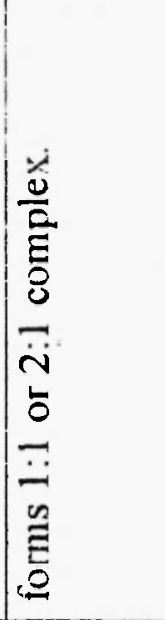 \\
\hline 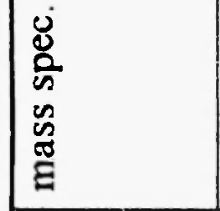 & 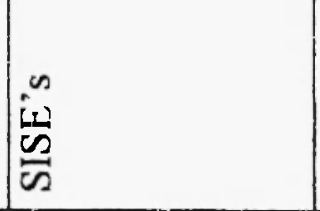 & ن & i & 治 \\
\hline $\mid \begin{array}{l}0 \\
\tilde{x} \\
\tilde{z} \\
z \\
\vdots \\
\vdots\end{array}$ & 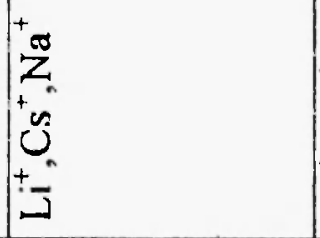 & $\begin{array}{l}x \\
x \\
+ \\
+\infty \\
z\end{array}$ & 禺 & $\bar{N}$ \\
\hline $\begin{array}{l}\infty \\
0 \\
\nabla^{-}\end{array}$ & $\theta$ & $\theta$ & $\nabla$ & $\Delta$ \\
\hline 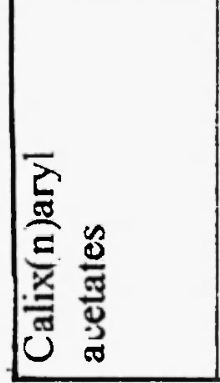 & 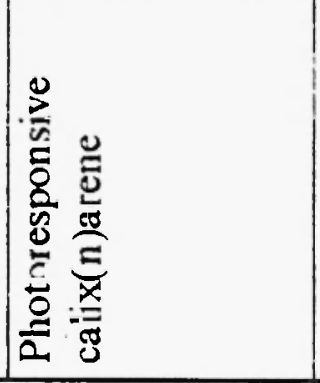 & 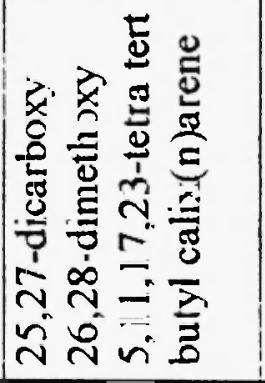 & 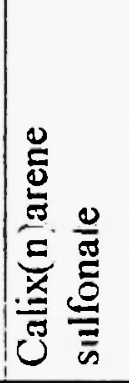 & 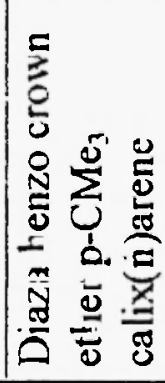 \\
\hline
\end{tabular}


Vol. 17, No. 2, 1998

Analytical Applications of Calixarenes

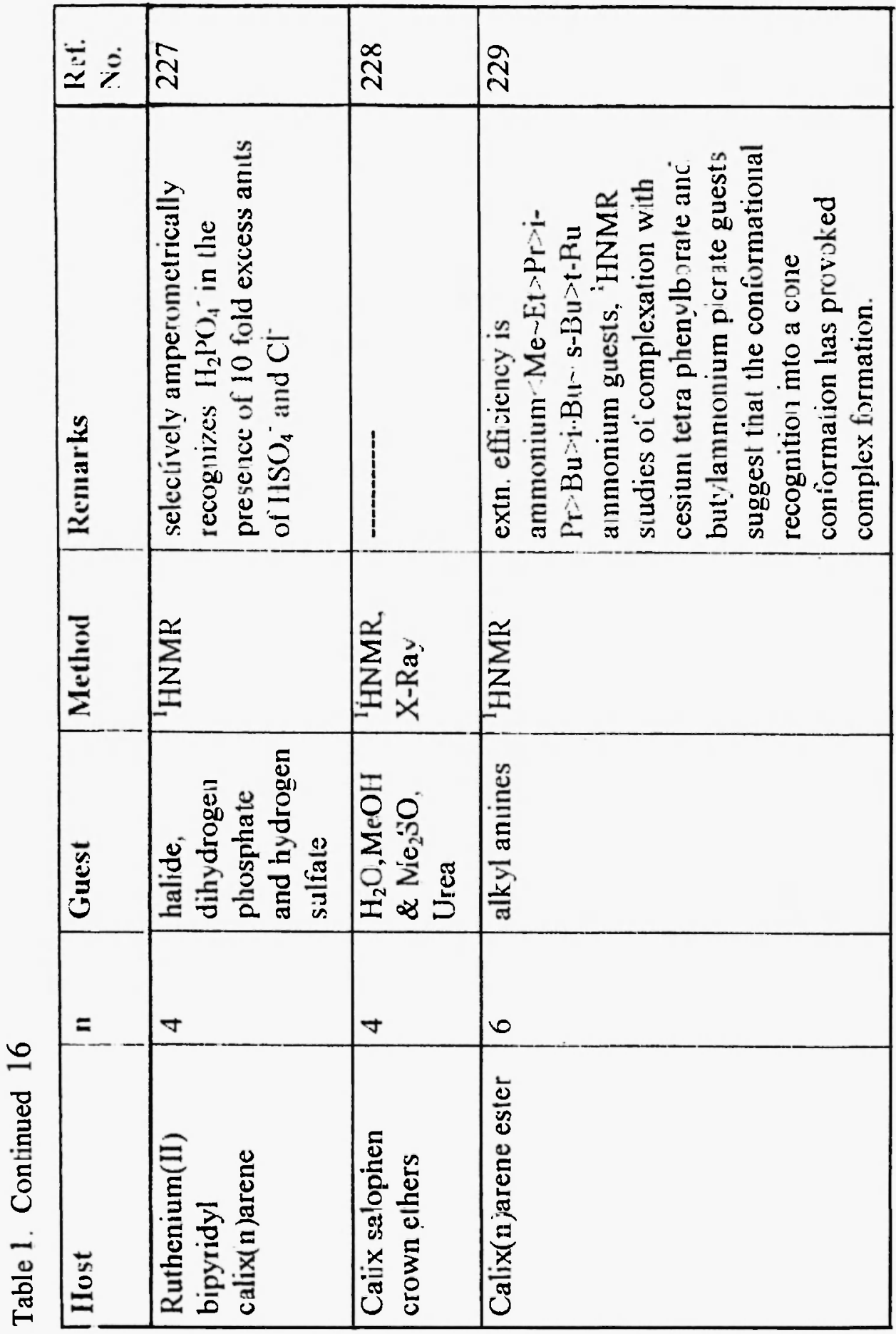




\begin{tabular}{|c|c|c|c|}
\hline 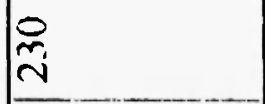 & $\vec{\sim}$ & $\tilde{N}_{1}$ & $\stackrel{\tilde{\sim}}{\sim}$ \\
\hline 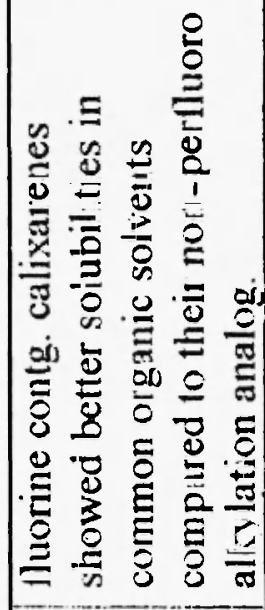 & 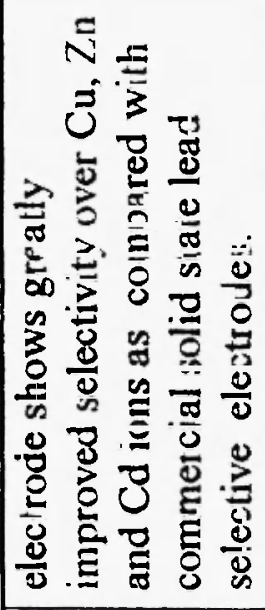 & 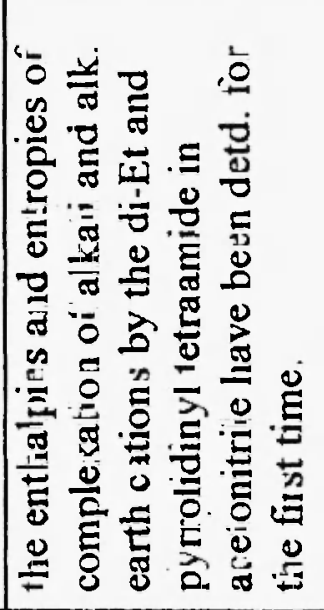 & 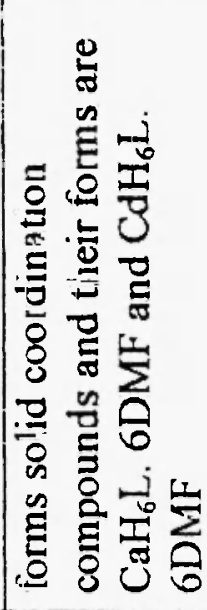 \\
\hline 1 & صు & 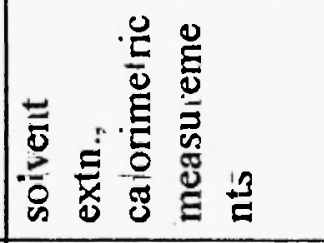 & 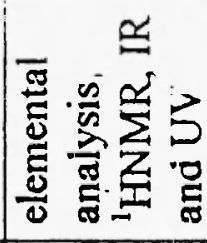 \\
\hline 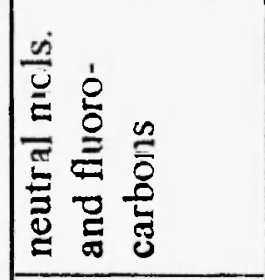 & $\frac{ \pm}{2}$ & 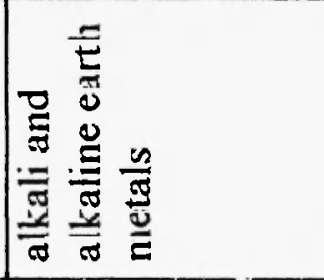 & 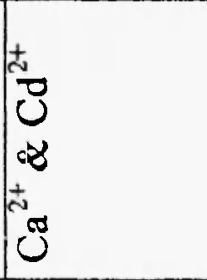 \\
\hline$\nabla$ & $\nabla$ & + & $\infty$ \\
\hline 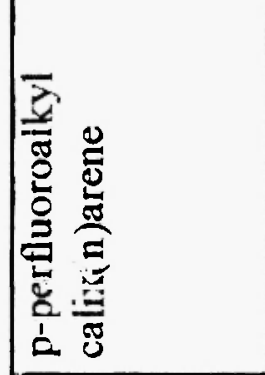 & 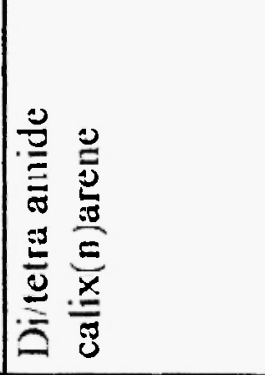 & 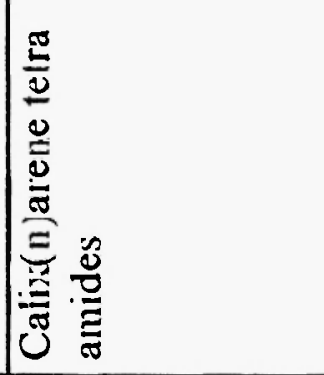 & 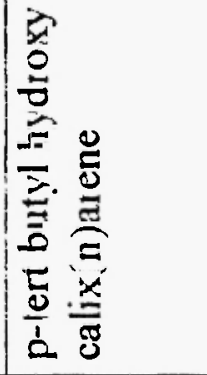 \\
\hline
\end{tabular}




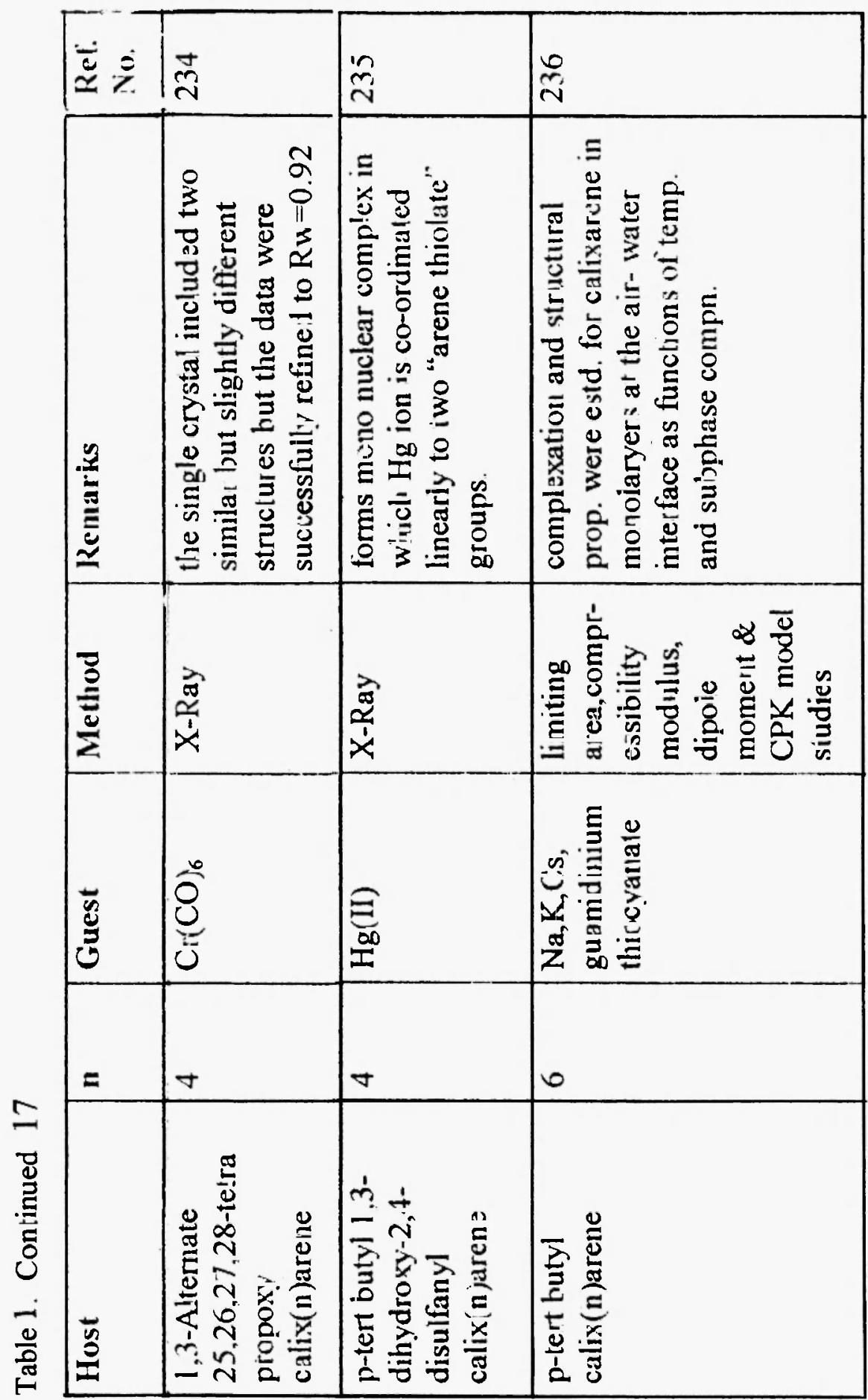




\begin{tabular}{|c|c|c|c|}
\hline$\stackrel{\sim}{\sim}$ & $\begin{array}{l}\infty \\
\ddot{\sim}\end{array}$ & 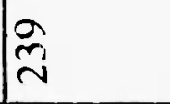 & 导 \\
\hline 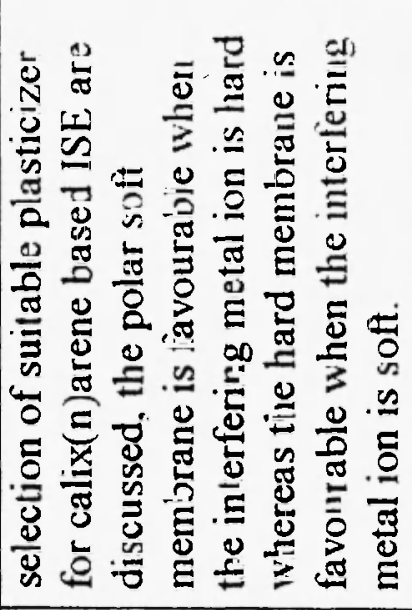 & 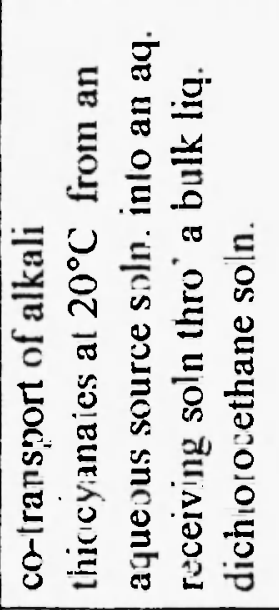 & 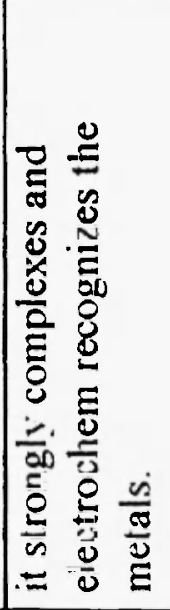 & 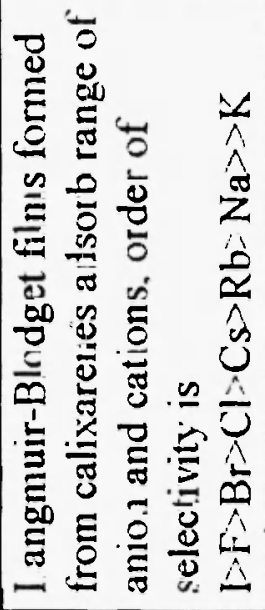 \\
\hline$\underline{\omega}$ & 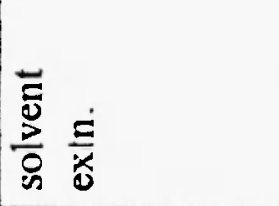 & 离 & 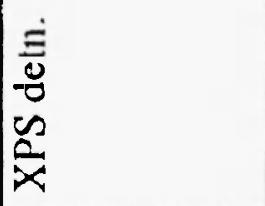 \\
\hline 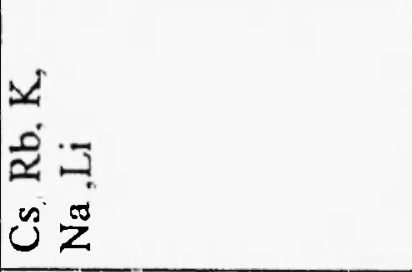 & 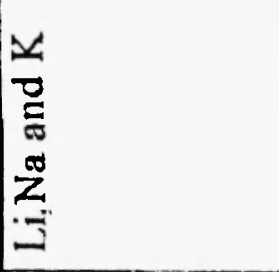 & 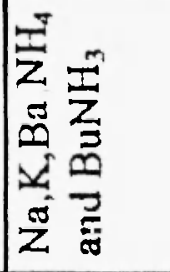 & 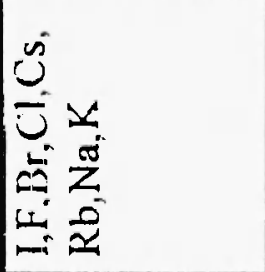 \\
\hline$\frac{\infty}{\frac{1}{\tau}}$ & $\begin{array}{l}\infty \\
0 \\
\sim \\
\sim\end{array}$ & $\nabla$ & $\infty$ \\
\hline 总 & 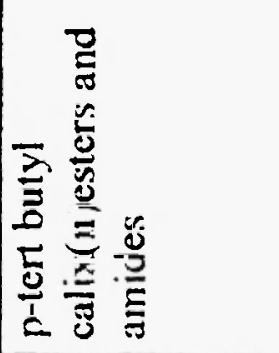 & 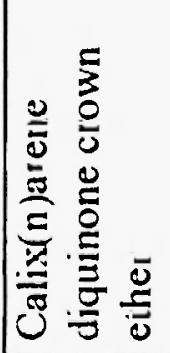 & 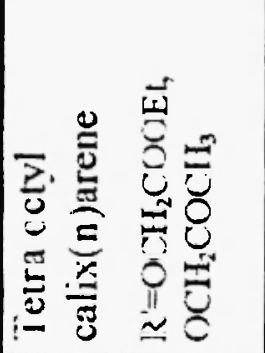 \\
\hline
\end{tabular}




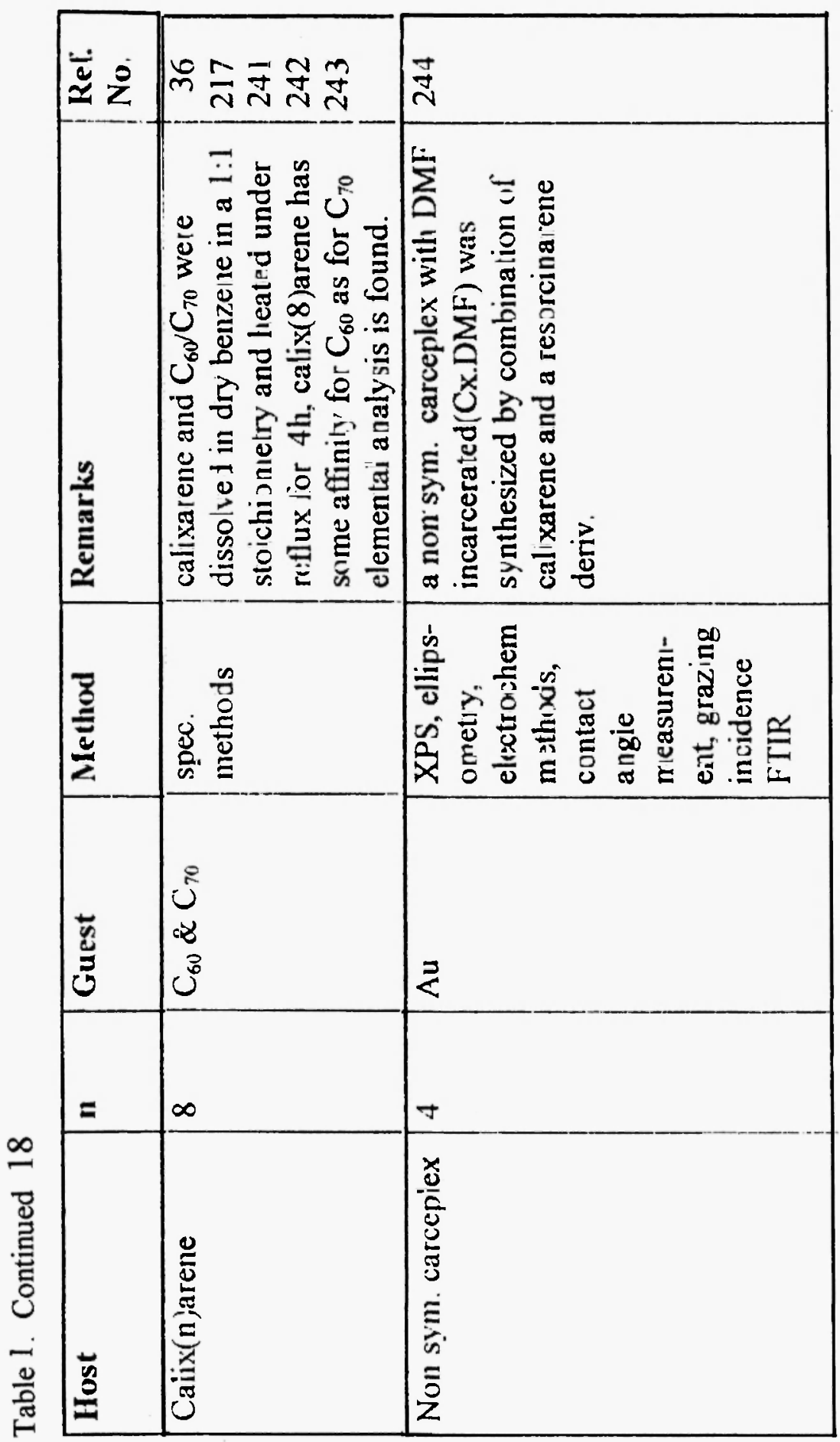




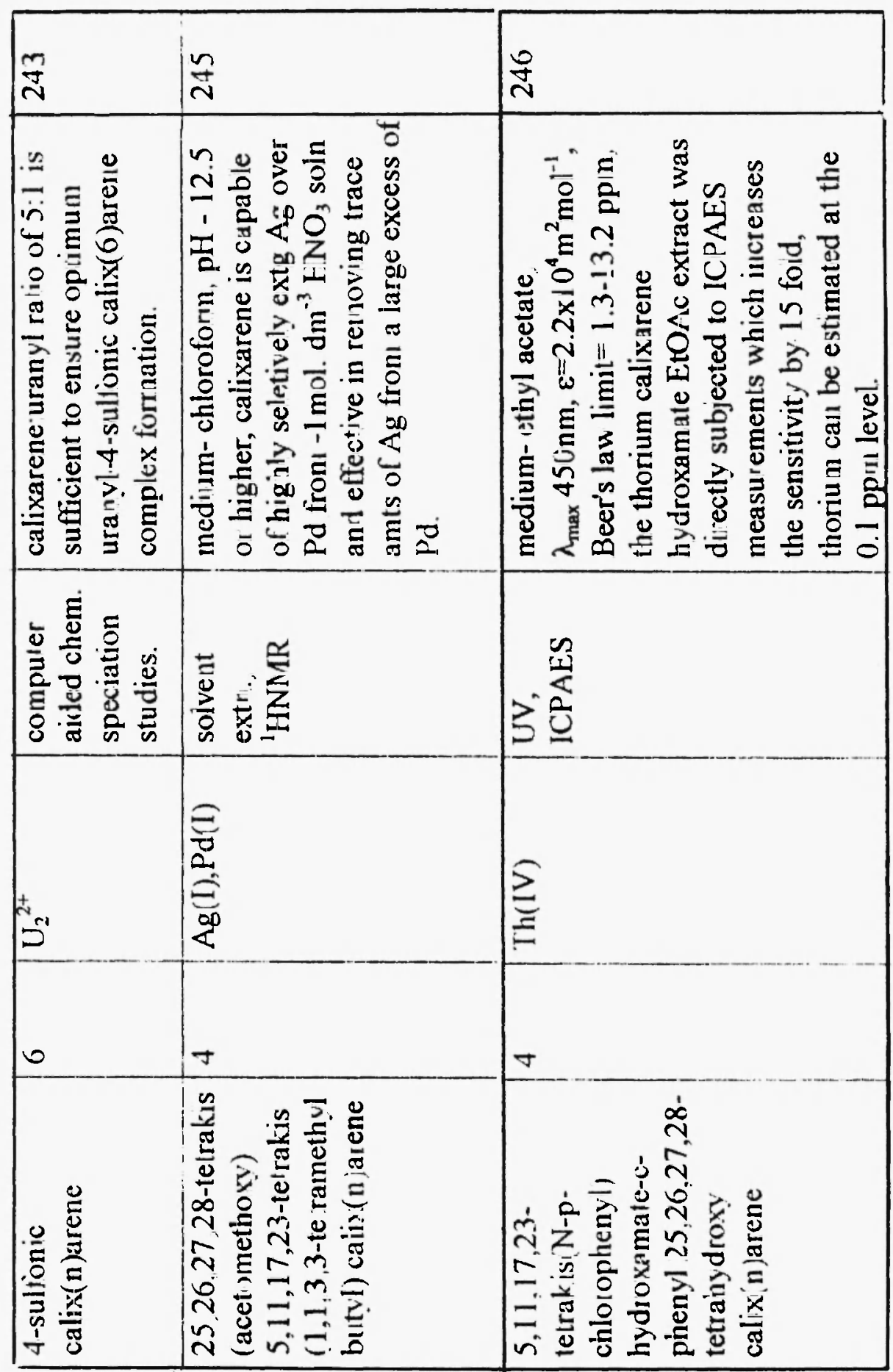




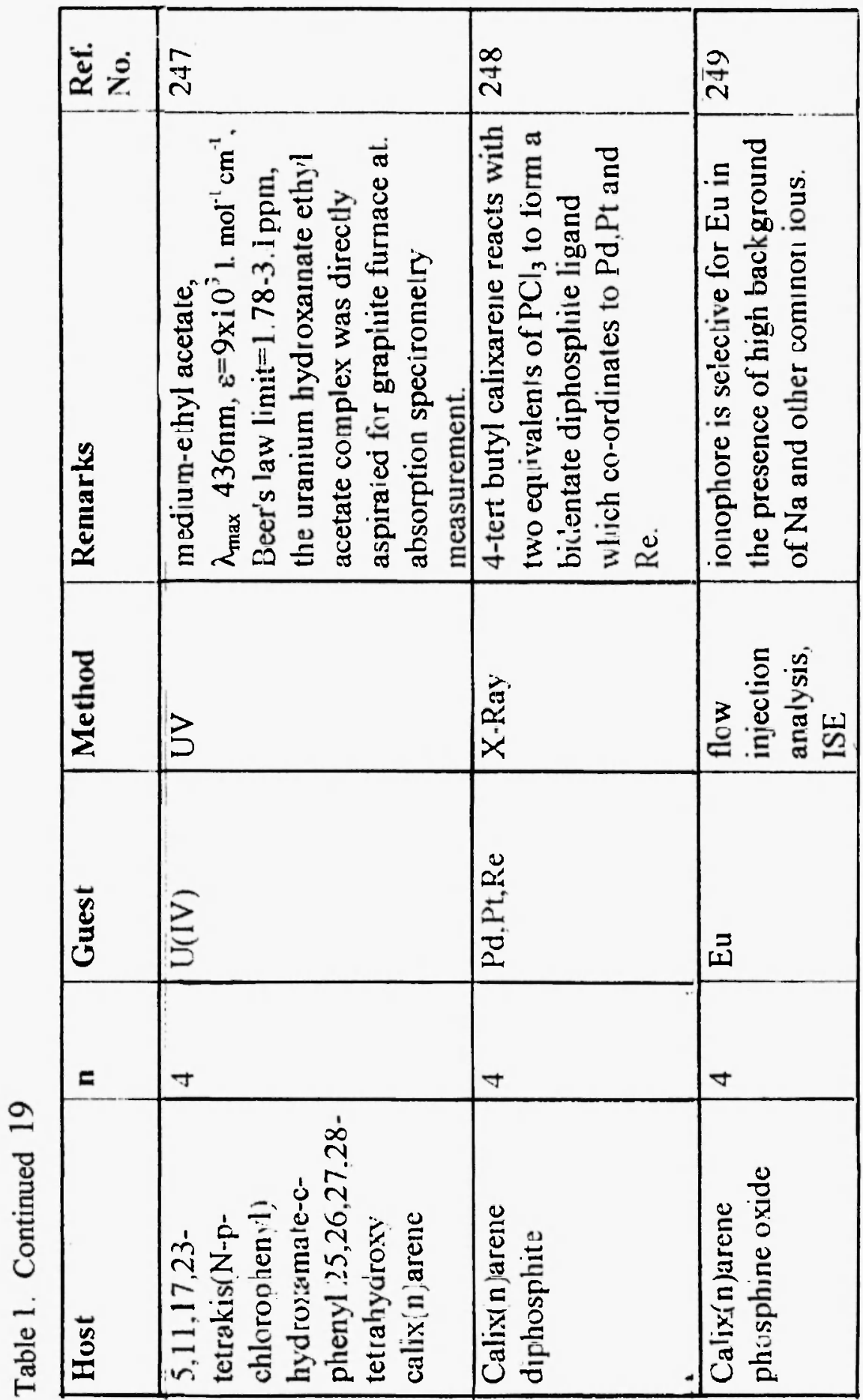




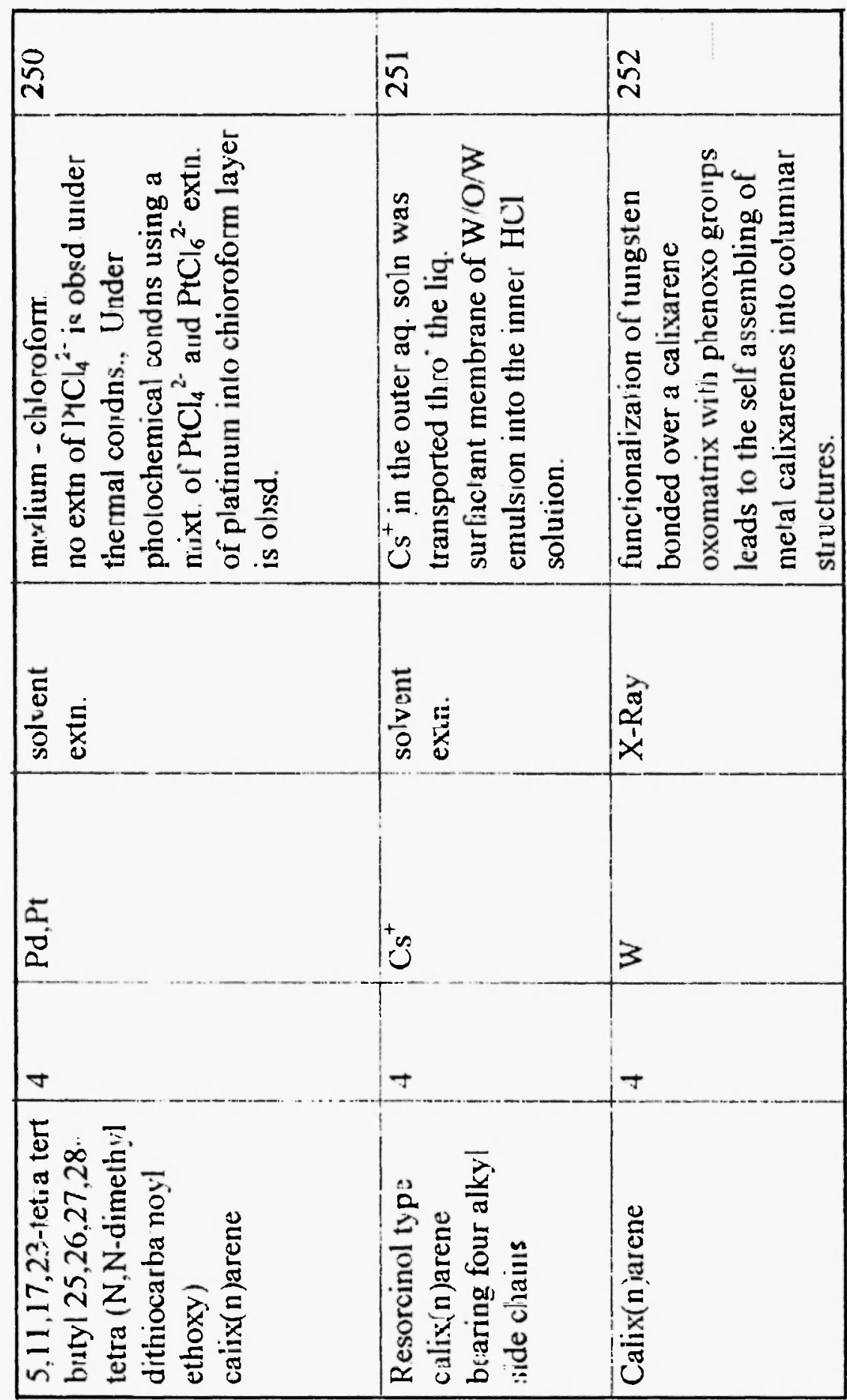




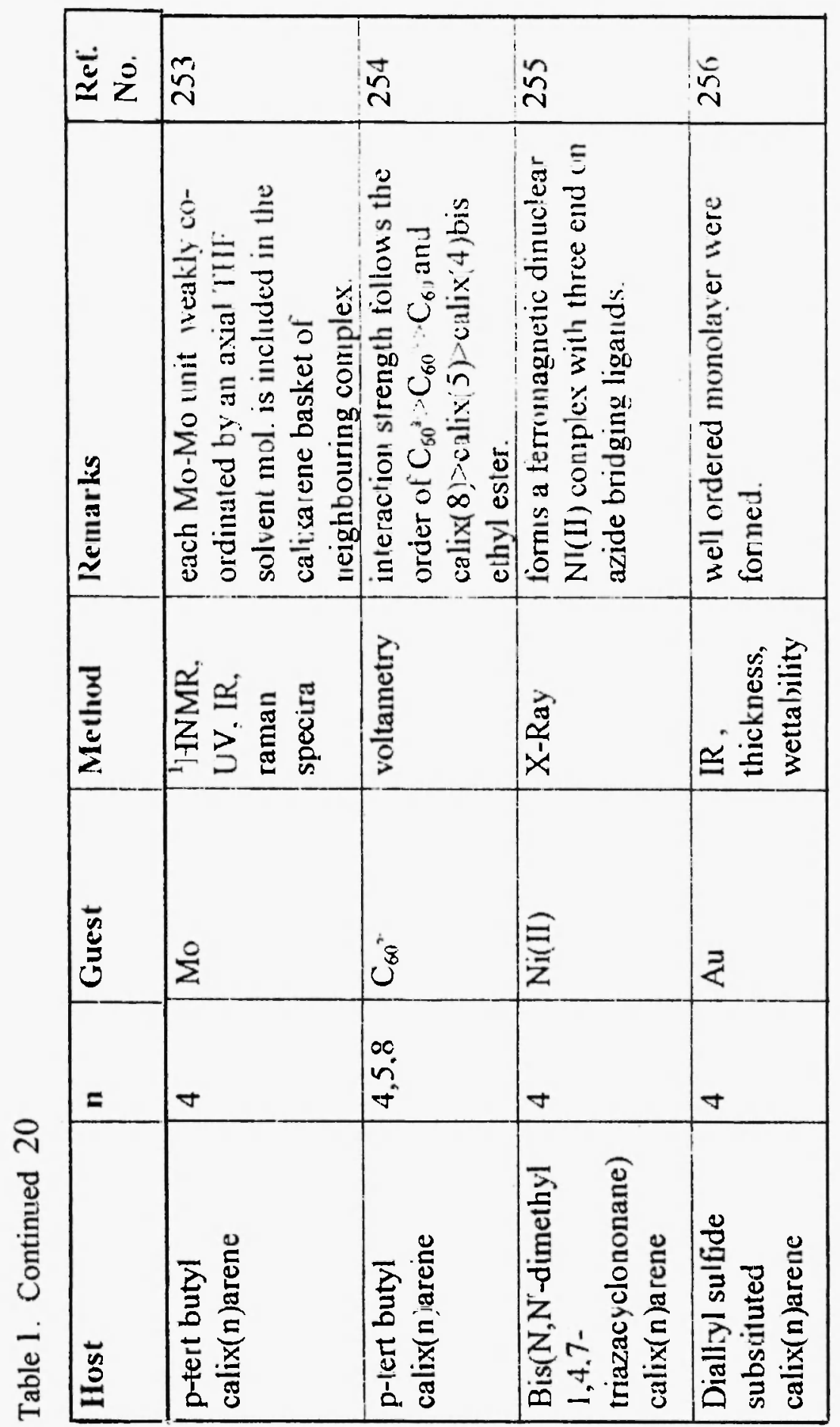




\begin{tabular}{|c|c|c|}
\hline$\sqrt[n]{\sim}$ & {$\left[\begin{array}{l}\infty \\
\infty \\
\cdots \\
N\end{array}\right.$} & $\tilde{\Omega}$ \\
\hline 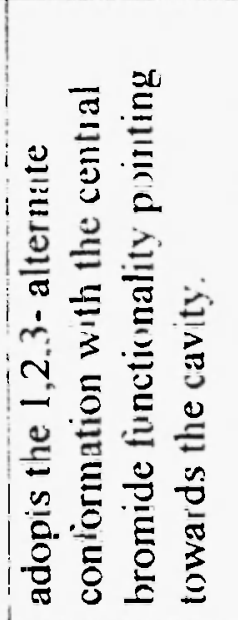 & 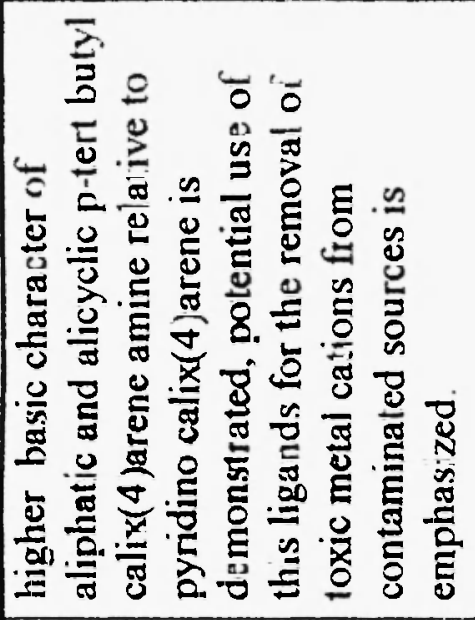 & 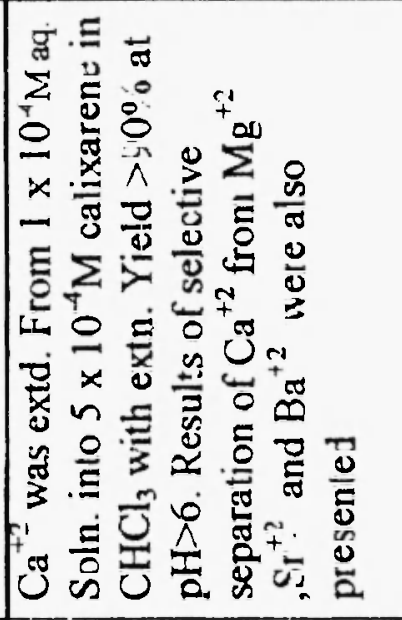 \\
\hline 吾 & 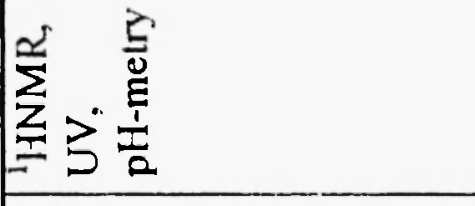 & 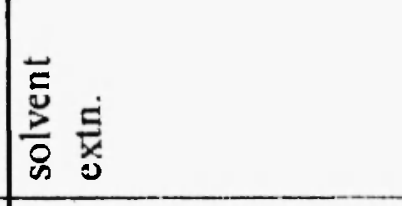 \\
\hline 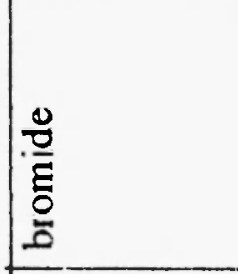 & 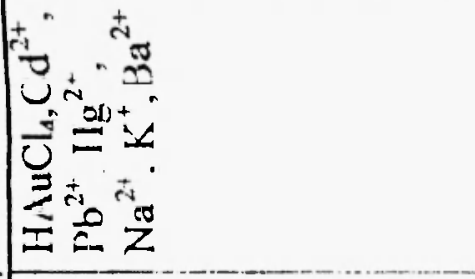 & 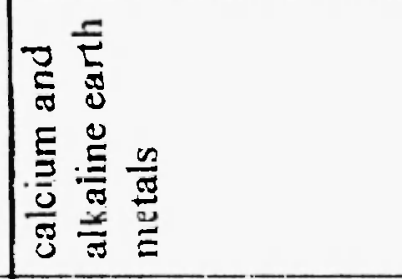 \\
\hline 10 & $\nabla$ & 10 \\
\hline 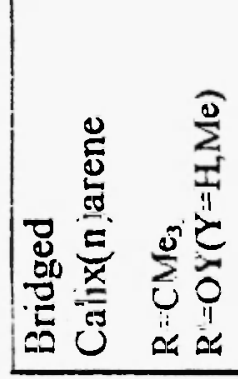 & 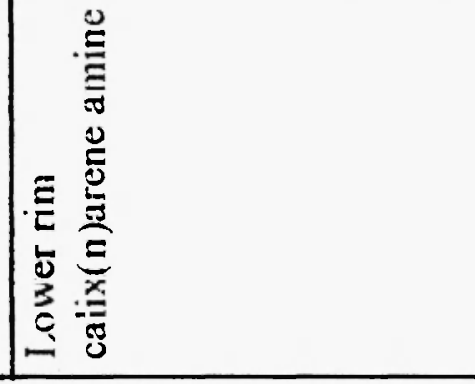 & 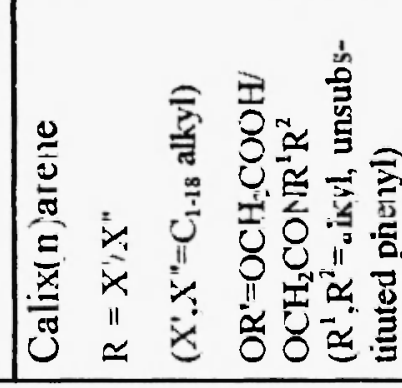 \\
\hline
\end{tabular}




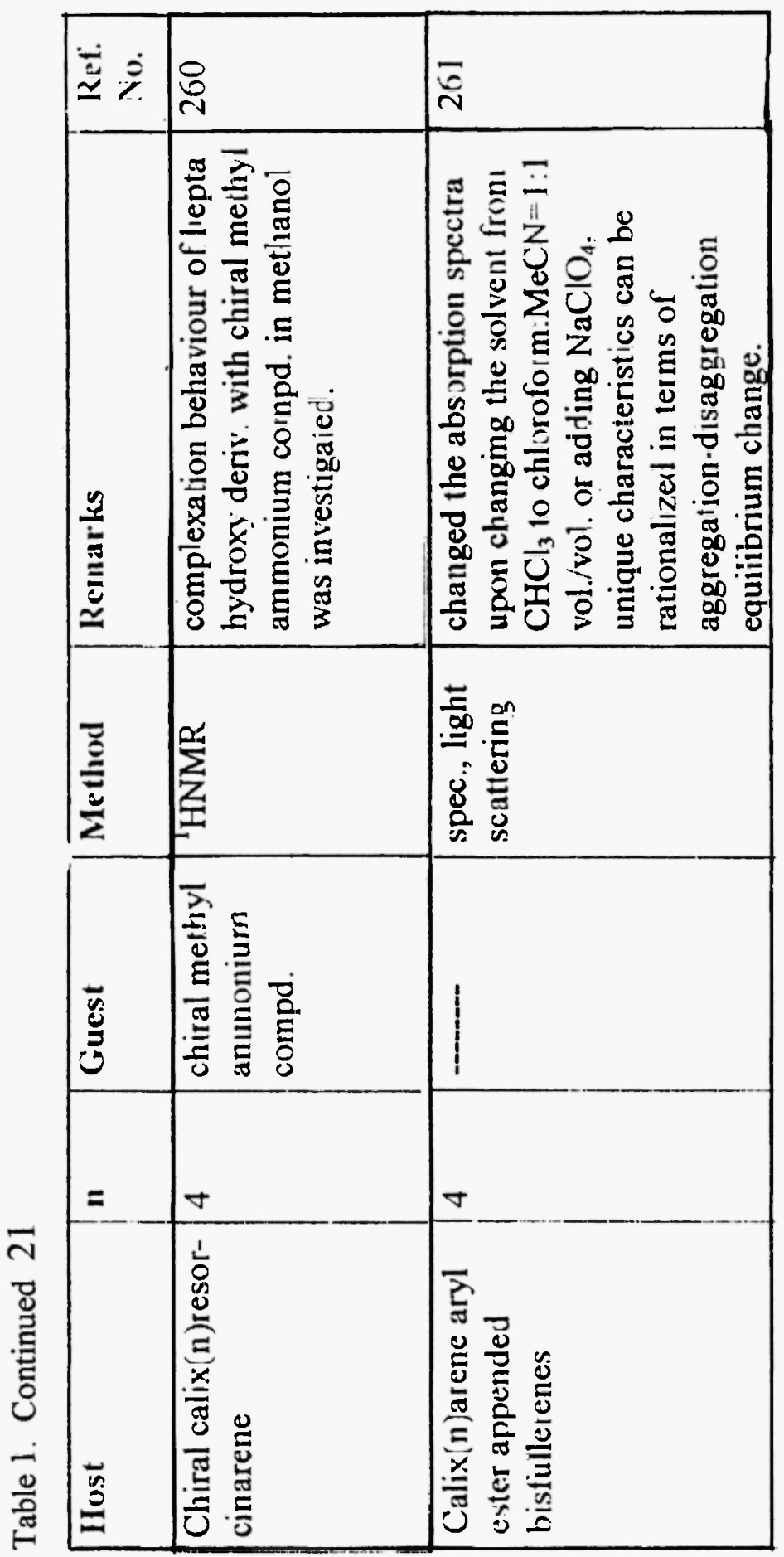




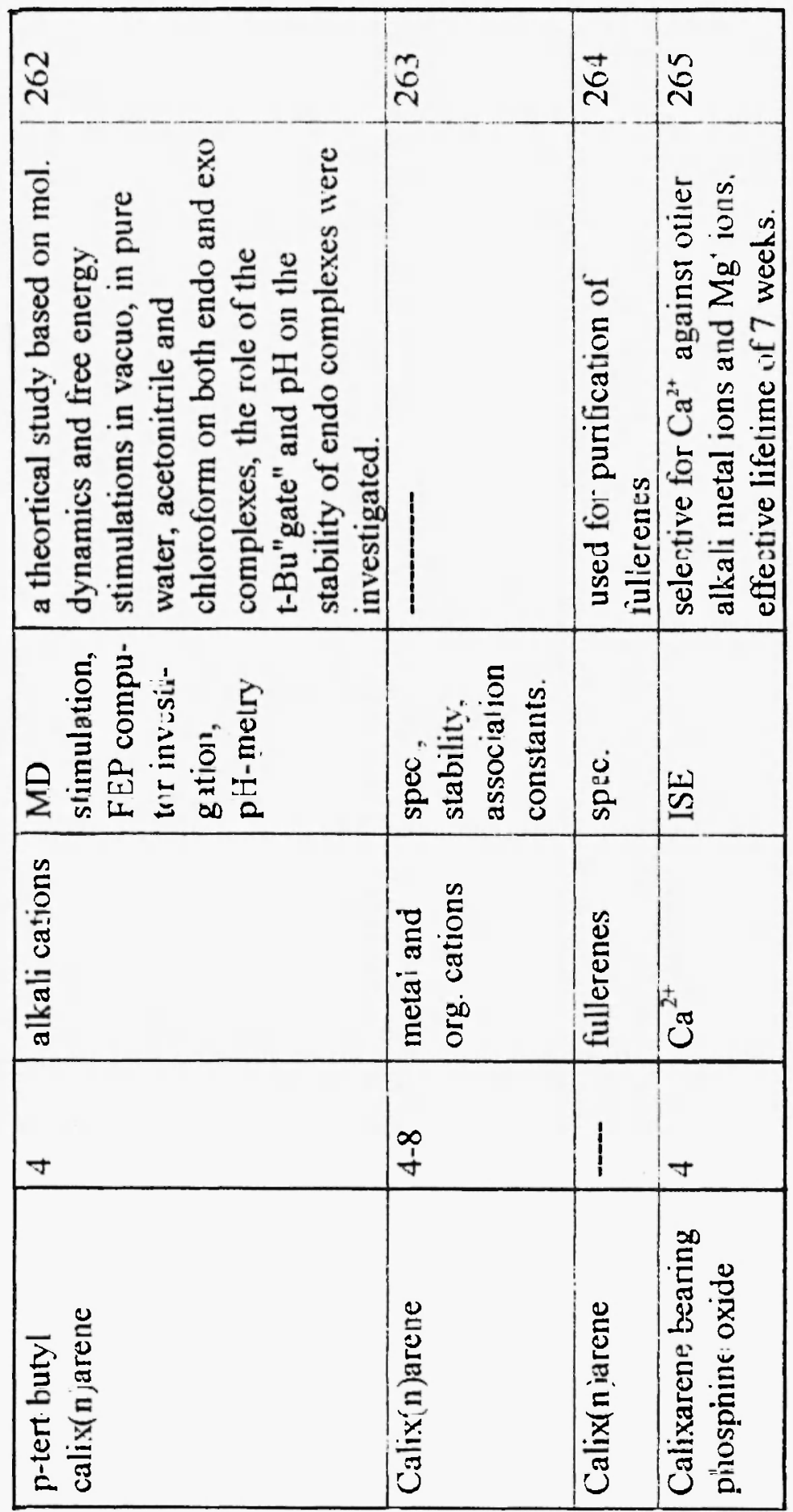




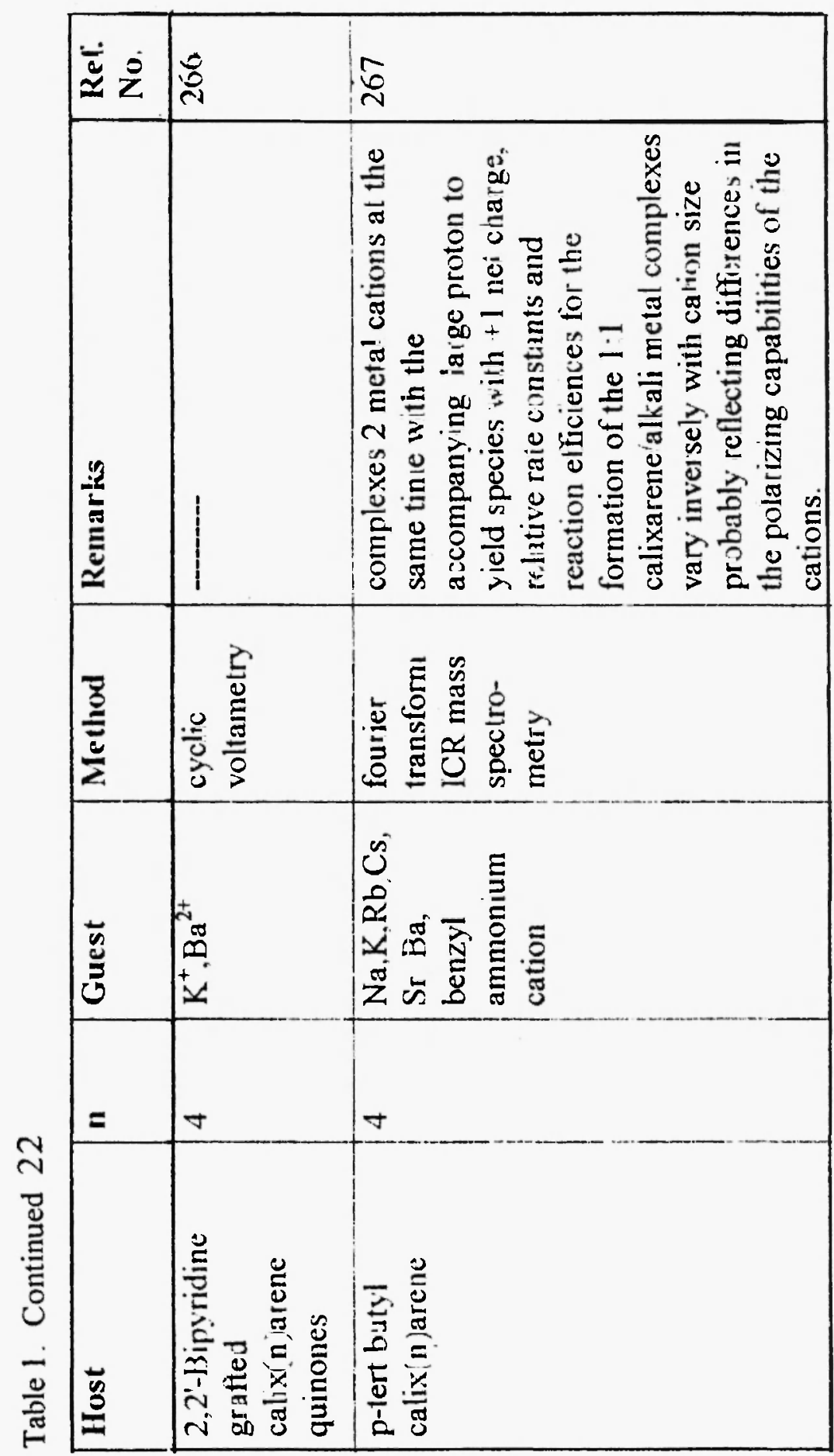




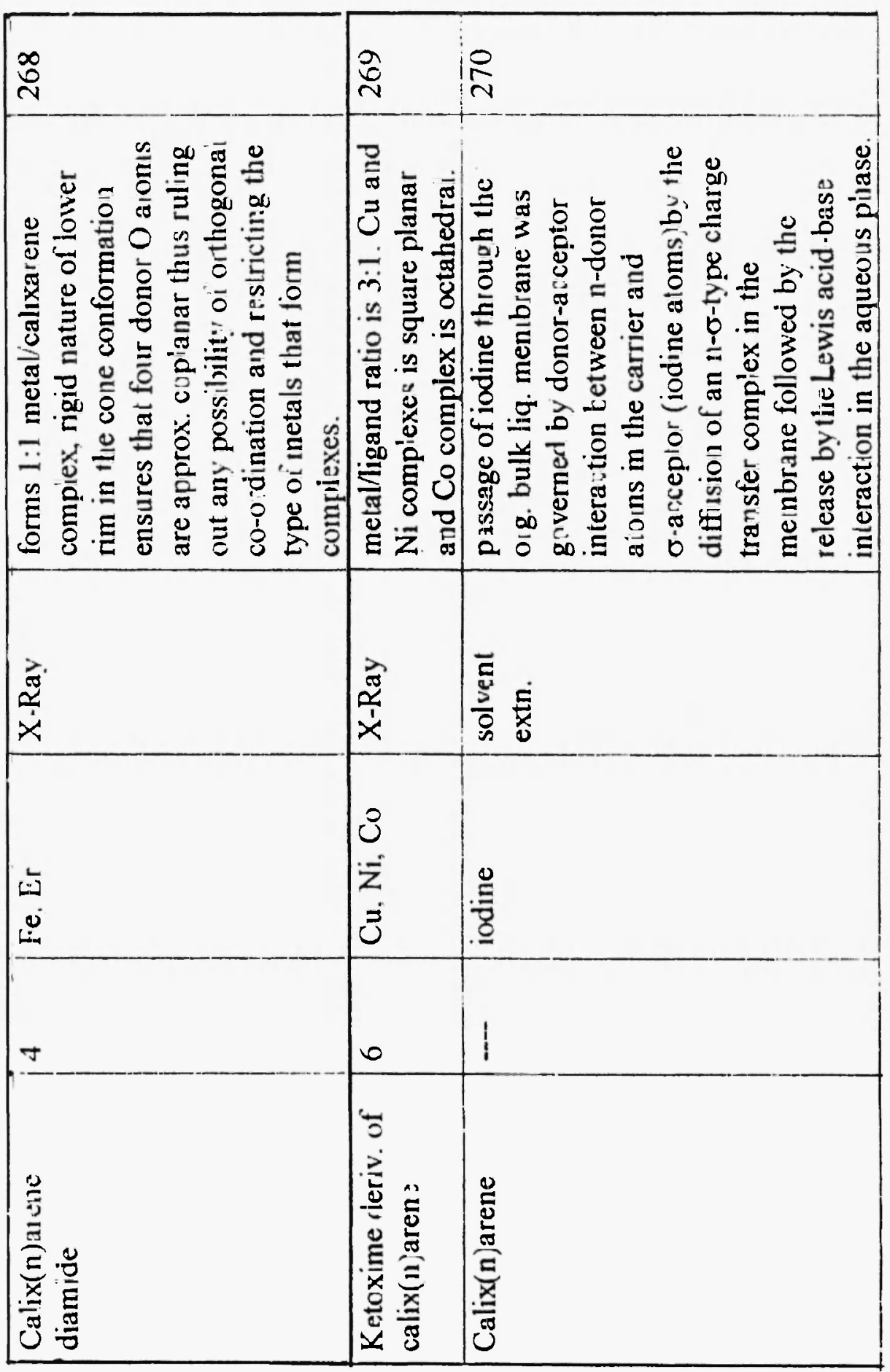




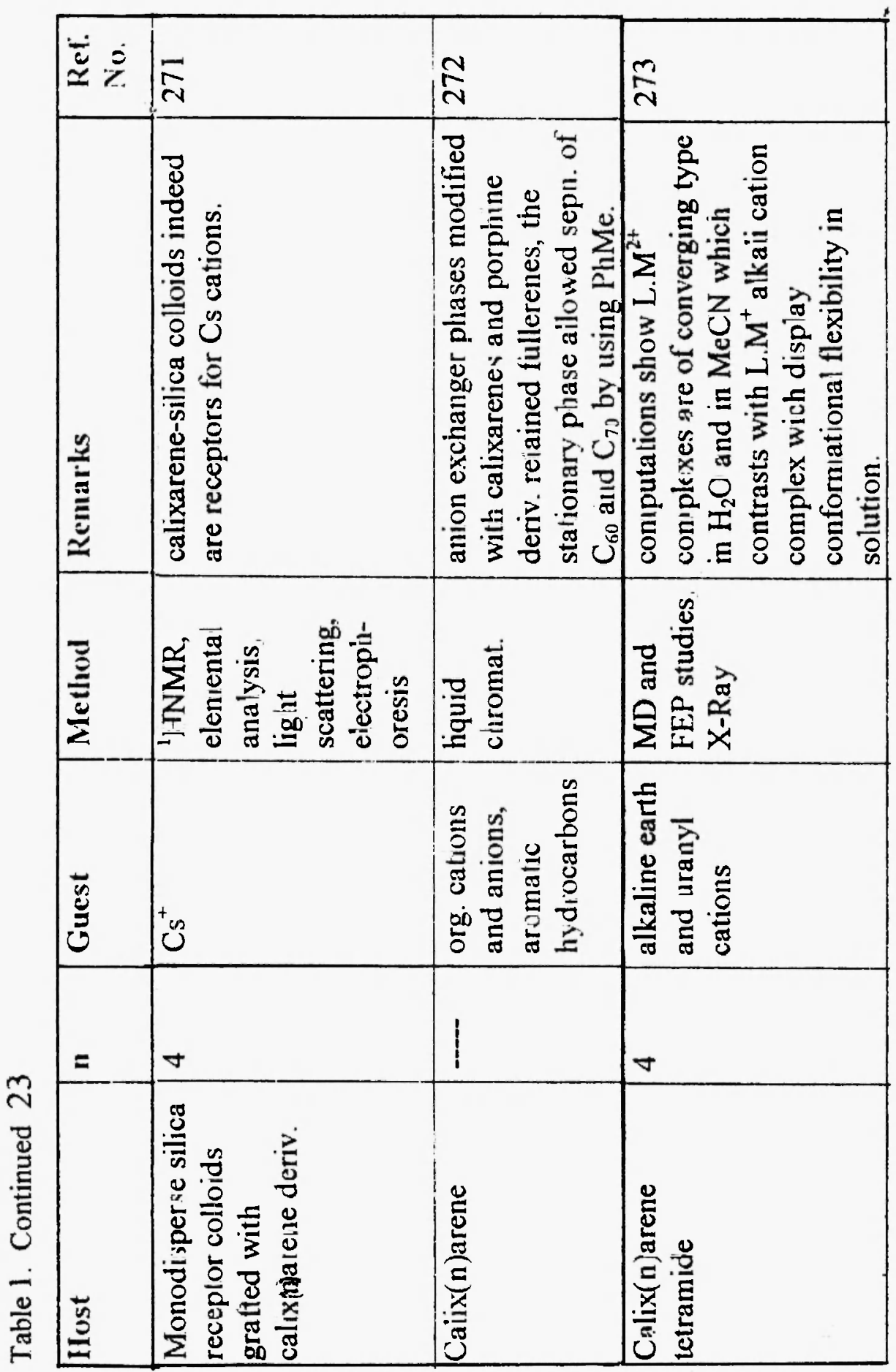




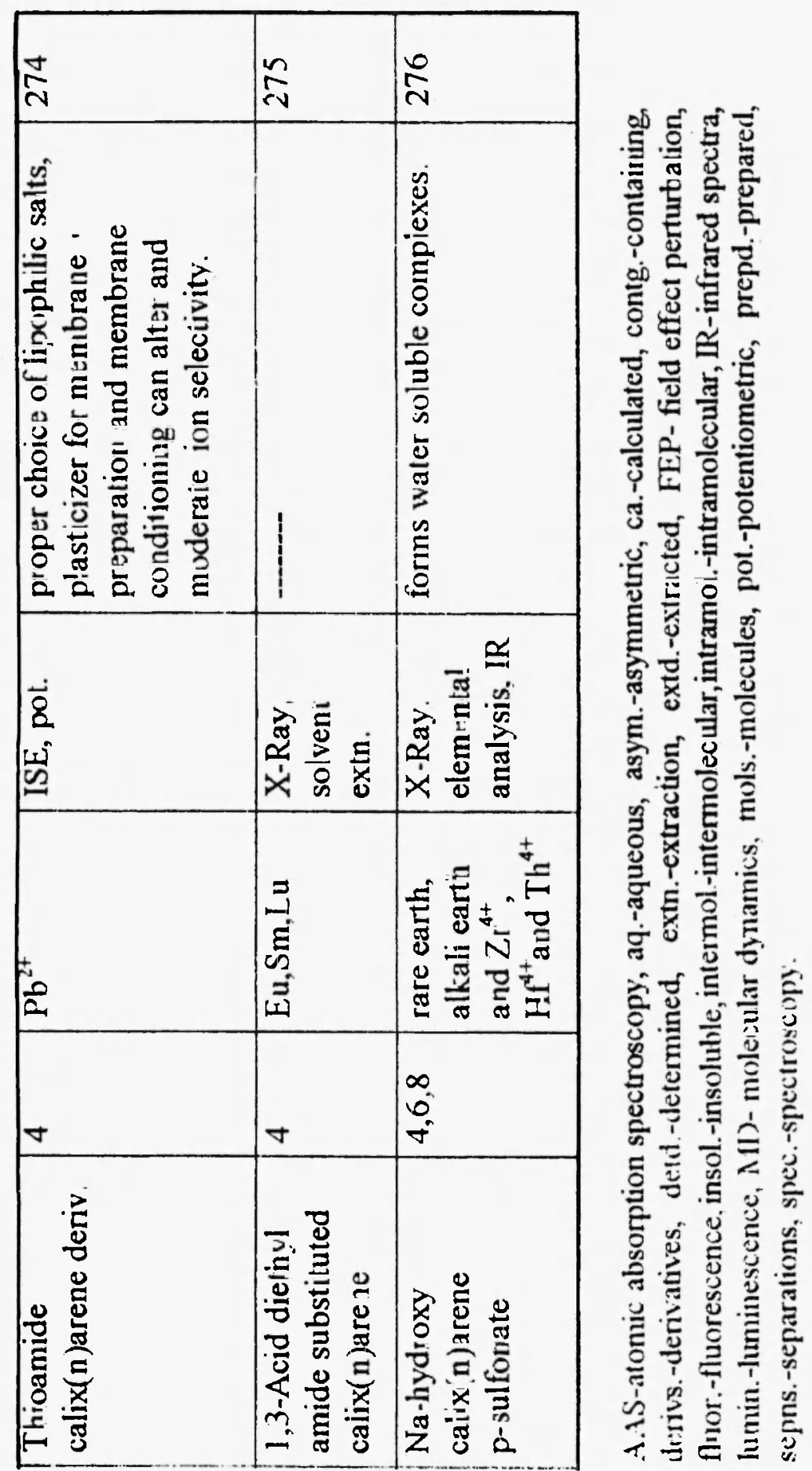




\section{REFERENCES}

1. A. Zinke, E. Ziegler, E. Martinowitz, H. Pichelmayer, M. Tomio, H. Wittmann-Zinke and S. Zwanziger, Ber, 77B, 264 (1944).

2. A. Baeyer, Ber., 5, 25 (1872).

3. A. Baeyer, Ber., 5, 280 (1872).

4. A. Baeyer, Ber, , 5, 1094 (1872).

5. L. Lederer, J. Prakt. Chemie, 50, 223 (1894).

6. O. Manasse, Ber., 27, 2409 (1894).

7. I. Blumer, British Patents 6,023 and 23,880 .

8. W.H. Storey, British Patent 8875.

9. A. Luft, British Patent 10,218.

10. L.H. Baekeland, U.S. Patent 942,699, October 1908.

11. L.H. Baekeland, Ind. Eng. Chem., 5, 506 (1913). For biographies of Baekeland cf. Refs. 12 and 13.

12. H.V. Potter, Chem. Ind., 242, 251 (1945).

13. J. Gills and R.E. Oesper, J. Chem. Educ., 41, 224 (1964).

14. T.S. Carswell, Phenoplasts, Interscience, New York, 1947.

15. A. Zinke, G. Zigeuner, K. Hössinger and G. Hoffman, Monatsh. Chem., 79, 438 (1948).

16. A. Zinke, R. Kretz, E. Leggewie, K. Hössinger, C. Hoffmann, P.W. von Ostwalden, E. Wiesenberger and M. Sobotka, Monatsh. Chem., 83, 1213 (1952).

17. A. Zinke, R. Ott and F.H. Garrana, Monatsh. Chem., 89, 135 (1958).

18. A. Zinke and R. Ott, Oesterr. Chem. Z., 55, 156 (1954).

19. J.B. Niederl and H.J. Vogel, J. Am. Chem. Soc., 62, 2512 (1940).

20. A.G.S. Högberg, Ph.D. Thesis, Royal Institute of Technology, Stockholm, Sweden, 1977.

21. B.T. Hayes and R.F. Hunter, Chem. Ind., 1956, 193; J. Appl. Chem., 8, 743 (1958).

22. H. Kämmerer, G. Happel and F. Caesar, Makromol. Chem., 162, 179 (1972). 
23. G. Happel, B. Mathiasch and H. Kämmerer, Makromol. Chem., 176, 3317 (1975).

24. H. Kämmerer and G. Happel, Makromol. Chem., 179, 1199 (1978).

25. H. Kämmerer, G. Happel, V. Böhmer and D. Rathay, Monatsh. Chem., 109, 767 (1978).

26. V. Bohmer, P. Chhim and H. Kämmerer, Makromol. Chem., 180, 2503 (1979).

27. H. Kămmerer and G. Happel, Makromol. Chem., 181, 2049 (1980).

28. H. Kämmerer and G. Happel, Monatsh. Chem., 112, 759 (1981).

29. H. Kåmmerer, G. Happel and B. Mathiasch, Makromol. Chem., 182, 1685 (1981).

30. J.W. Cornforth, P.D. Hart, G.A. Nicholls, R.J.W. Rees and J.A. Stock, Br. J. Pharmacol., 10, 73 (1955).

31. J.H. Munch, Makromol. Chem., 69, 178 (1977).

32. R.S. Buriks, A.R. Fauke and J.H. Munch, U.S. Patent 4,259,464; filed 1976, issued 1981.

33. R.S. Buriks, A.R. Fauke and F.E. Mange, U.S. Patent 4,098,717; filed 1977, issued 1978.

34. C.D. Gutsche, T.C. Kung and M.L. Hsu, Abstracts 11th Midwest Regional Meeting Am. Chem. Soc., Carbondale, IL, No. 517, 1975.

35. C.D. Gutsche and R. Muthukrishnan, J. Org. Chem., 43, 4905 (1978).

36. C.D. Gutsche, B. Dhawan, K.H. No and R. Muthukrishnan, J. Am. Chem. Soc., 103, 3782 (1981).

37. C.D. Gutsche, Acc. Chem. Res., 16, 161 (1983).

38. C.D. Gutsche, Top. Curr. Chem., 1984, 123.

39. G.D. Andreetti, R. Ungaro and A. Pochini, J. Chem. Soc., Chem. Commun., 1979, 1005.

40. G.D. Andreetti, R. Ungaro and A. Pochini, J. Chem. Soc., Chem. Commun., 1981, 533.

41. M. Coruzzi, G.D. Andreetti, V. Bocchi, A. Pochini and R. Ungaro, $J$. Chem. Soc., Perkin Trans., 1982 (9), 1133.

42. M. Iqbal, T. Mangiafico and C.D. Gutsche, Tetrahedron, 43, 4917 (1987). 
43. V. Bocchi, D. Foina, A. Pochini and R. Ungaro, Tetrahedron, 38, 373 (1982).

44. C.D. Gutsche, B. Dhawan, J.A. Levine, K.H. No and L.J. Bauer, Tetrahedron, 39, 409 (1983).

45. C.D. Gutsche and L.G. Lin, Tetrahedron, 42, 1633 (1986).

46. J.W. Cornforth, E.D. Morgan, K.T. Potts and R.J.W. Rees, Tetrahedron, 29, 1659 (1973).

47. C.D. Gutsche and G.F. Stanley, unpublished works.

48. A. Arduini, A. Pochini, S. Reverberi and R. Ungaro, J. Chem. Soc., Chem. Commun., 1984, 981.

49. R. Ungaro, A. Pochini and G.D. Andreetti, J. Inclusion Phenom., 2, 199 (1984).

50. M.A. Mckervey, E.M. Seward, G. Ferguson, B. Ruhl and S.J. Harris, J. Chem. Soc., Chem. Commun., 1985, 388.

51. M.A. Mckervey, E.M. Seward, G. Ferguson and B. Ruhl, J. Org. Chem., 51, 3581 (1986).

52. G. Ferguson, B. Kaitner, M.A. Mckervey and E.M. Seward, J. Chem. Soc., Chem. Commun., 1987, 584.

53. S.K. Chang, S.K. Kwon and I. Cho, Chem. Letts., 1987, 947.

54. C.D. Gutsche, M. Iqbal, K.C. Nam, K.A. See and I. Alam, Pure Appl. Chem., 60, 483 (1988).

55. V. Böhmer, D. Rathay and H. Kämmerer, Org. Prep. Proced. Int., 10, 113 (1978).

56. C.D. Gutsche and J.A. Levine, J. Am. Chem. Soc., 104, 2652 (1982).

57. C.D. Gutsche, J.A. Levine and P.K. Sujeeth, J. Org. Chem., 50, 5802 (1985).

58. S. Shinkai, S. Mori, T. Tsubaki, T. Sone and O. Manabe, Tetrahedron Lett., 25, 5315 (1984).

59. S. Shinkai, K. Araki, T. Tsubaki, T. Arimura and O. Manabe, J. Chem. Soc., Perkin Trans. I, 1987, 2297.

60. (a) S. Shinkai, T. Tsubaki, T. Sone and O. Manabe, Tetrahedron Lett., 26, 3343 (1985); (b) S. Shinkai, H. Koreishi, K. Ueda and O. Manabe, J. Chem. Soc., Chem. Commun., 1986, 233. 
61. (a) K.C. Nam, Ph.D. Thesis, Washington Univ., St. Louis, 1987;

(b) K. No and Y. Noh, Bull. Korean Chem. Soc., 7, 314 (1986).

62. C.D. Gutsche and K.C. Nam, J. Am. Chem. Soc., 110, 6153 (1988).

63. C.D. Gutsche and I. Alam, Tetrahedron, 44 (15), 4689 (1988).

64. A. Arduini, A. Casnati, A. Pochini and R. Ungaro, Fifth Intern. Symp. Inclusion Phenomena \& Molecular Recognition, Orange Beach, Ala, 1988; p. H13.

65. A.A. Moshfegh, R. Badri, M. Hojjatie, M. Kaviani, B. Naderi, A.H. Nazmi, M. Ramezanian, B. Roozpeikar and G.H. Hakimelahi, Helv. Chim. Acta, 65, 1221 (1982).

66. A.A. Moshfegh, B. Mazandarani, A. Nahid and G.H. Hakimelahi, ibid, 65, 1229 (1982).

67. A.A. Moshfegh, E. Beladi, L. Radnia, A.S. Hosseini, S. Tofigh and G.H. Hakimelahi, ibid., 65, 1264 (1982).

68. A. Ninagawa, K. Cho and H. Matsuda, Makromol. Chem., 186, 1329 (1985).

69. L. Rosyk, Ph.D. Thesis, Washington Univ., St. Louis, 1986; p. 29.

70. G.D. Andreetti, O. Ori, F. Uguzzoli, C. Alfieri, A. Pochini and R. Ungaro, J. Inclusion Phenom., 6, 523 (1988).

71. A.W. Coleman, S.G. Bott and J.L. Atwood, J. Inclusion Phenom., 4 (3), 247 (1986).

72. J.R. Moran, S. Karbach and D.J. Cram, J. Am. Chem. Soc., 104, 5826 (1982).

73. D.W. Chaser, J. Org. Chem., 50, 545 (1985).

74. T. Asao, S. Ito and N. Morita, Tetrahedron Lett., 29, 2839 (1988).

75. G.D. Andreetti, A. Pochini and R. Ungaro, J. Chem. Soc., Perkin Trans. II., 1983, 1773.

76. T. Komoto, I. Ando, Y. Nakamoto and S. Ishida, J. Chem. Soc., Chem. Commun., 1988, 135.

77. C.D. Johnson and F.A. Bovey, J. Chem. Phys., 29, 1012 (1958).

78. S.G. Bott, A.W. Coleman and J.L. Atwood, J. Am. Chem. Soc., 108 (7), 1709 (1986).

79. S.G. Bott, A.W. Coleman and J.L. Atwood, J. Am. Chem. Soc., 110, 610 (1988). 
80. D.N. Reinhoudt, P.J. Dijkstra, P.J.A. in't Veld, K.E. Bugge, S. Harkema, R. Ungaro and E. Ghidini, J. Am. Chem. Soc., 109, 4761 (1987).

81. D.J. Cram, S. Karbach, H.E. Kim, C.B. Knobler, E.F. Maverick, J.L. Ericson and R.C. Helgeson, J. Am. Chem. Soc., 110, 2229 (1988).

82. A. Ninagawa and H. Matsuda, Makromol. Chem., Rapid Commun., 3, 65 (1982).

83. L. Rizzoli, G.D. Andreetti, R. Ungaro and A. Pochini, J. Mol. Struct., 82, 133 (1982).

84. R. Ungaro, A. Pochini, G.D. Andreetti and V. Sangermano, J. Chem. Soc., Perkin Trans. II., 1984, 1979.

85. D.J. Cram, K.D. Stewart, I. Goldberg and K.N. Trueblood, J. Am. Chem. Soc., 107, 2574 (1985).

86. I. Goldberg, J. Inclusion Phenom., 4, 191 (1986).

87. Y. Nakamoto and S. Ishida, Makromol. Chem., Rapid Commun., 3, 705 (1982).

88. J. Uzawa, S. Zushi, Y. Kodama, Y. Fukuda, K. Nishihata , K. Umemura, M. Nishio and M. Hirota, Bull. Chem. Soc. Jpn., 53, 3623 (1986).

89. G.D. Andreetti, G. Calestani, F. Ugozzoli, A. Arduini, E. Ghidini, A. Pochini and R. Ungaro, J. Inclusion Phenom., 5, 123 (1987).

90. D.J. Cram, S. Karbach, Y.H. Kim, L. Baczynskyj, K. Marti, R.M. Sampson and G.W. Kalleymeyn, J. Am. Chem. Soc., 110, 2554 (1988).

91. R.M. Izatt, J.D. Lamb, R.T. Hawkins, P.R. Brown, S.R. Izatt and J.J. Christensen, J. Am. Chem. Soc., 105, 1782 (1983).

92. S.R. Izatt, R.T. Hawkins, J.J. Christensen and R.M. Izatt, J. Am. Chem. Soc., 107, 63 (1985).

93. H. Goldmann, W. Vogt, E. Paulus and V. Böhmer, J. Am. Chem. Soc., 110, 6811 (1986).

94. R. Ungaro, A. Pochini, G.D. Andreetti and P. Domiano, J. Inclusion Phenom., 3, 35 (1985).

95. G. Calestani, F. Ugozzoli, A. Arduini, E. Ghidini and R. Ungaro, J. Chem. Soc., Perkins Trans. I., 1987, 344. 
96. A. Arduini, A. Pochini, S. Reverberi and R. Ungaro, Tetrahedron, 42, 2089 (1986).

97. S.K. Chang and I. Cho, Chem. Lett., 1984, 477.

98. S.K. Chang, J. Chem. Soc., Perkins Trans. I., 1986, 211.

99. D.J. Cram, G.M. Lein, T. Kaneda, R.C. Helgeson, M.E. Knobler and K.N. Trueblood, J. Am. Chem. Soc., 103, 6228 (1981).

100. J. Bauer and C.D. Gutsche, J. Am. Chem. Soc., 107, 6063 (1987).

101. C.D. Gutsche, M. Iqbal and L. Alam, J. Am. Chem. Soc., 109, 4314 (1987).

102. H.A. Benersi and J.H. Hildebrand, J. Am. Chem. Soc., 71, 2703 (1949).

103. Y. Aoyama, S. Sugihara and Y. Tanaka, submitted for publication.

104. J.L. Atwood, A.W. Coleman, H. Zhang and S.G. Bott, J. Inclusion Phenom., Mol. Recognit. Chem., 7(2), 203 (1989).

105. J.L. Atwood, F. Hamada, K.D. Robinson, G.W. Orr and R.L. Vincent, Nature, 349, 683 (1991).

106. T.M. Liang and K.K. Laali, Chem. Ber., 124(11), 2637 (1991).

107. (a) J.M. Harrowfield, M.I. Ogden, W.R. Richmond and A.H. White, J. Chem. Soc., Chem. Commun., 1991, 1159. (b) J.C.G. Bunzli and J.M. Harrowtield, in: Topics in Inclusion Science, Vol. 3. Calixarenes - A Versatile Class of Macrocyclic Compounds, J. Vicens and V. Böhmer (eds.), Kluwer, Dordrecht, 1991; p. 211.

108. S. Shinkai, ibid, p. 173.

109. V. Böhmer and J. Vicens, ibid., p. 39.

110. R. Perrin and S.J. Harris, ibid, p. 235.

111. S.J. Harnis, M.A. Mckervey, D.P. Melody, J. Woods and J. Rooney, Eur. Pat. Appl. EP 151,527, 1984.

112. S.J. Harris, M.A. Mckervey, D.P. Melody and J. Woods, Eur. Pat. Appl. EP 196,881, 1986.

113. M.A. Mckervey, U.S. Patent 4,622,414, 1986.

114. R.G. Leonard and S.J. Harris, U.S. Patent 4,695,615, 1987.

115. R. Izatt, J. Christensen and R. Hawkins, U.S. Patent 4,477,377, 16 Oct. 1984, Appl. 389,402, 17 June 1982, 5 pp.

116. A. Mangia, A. Pochini, R. Ungaro and G.D. Andreetti, Anal. Lett., 16, 1027 (1983). 
117. R. Ungaro and A. Pochini, in: Topics in Inclusion Science, Vol. 3. Calixarenes $-A$ Versatile Class of Macrocyclic Compounds, J. Vicens and V. Bohmer (eds.), Kluwer, Dordrecht, 1991; p. 127.

118. A.M. Cadogan, D. Diamond, S. Cremin, A.M. Mckervey and S.J. Harris, Anal. Proc., 28, 13 (1991).

119. (a) M. Telting-Diaz, D. Diamond and M.R. Smyth, Anal. Chem. Acta, 251, 149 (1991); (b) D. Diamond, G. Svehla, E.M. Seward and M.A. Mckervey, Anal. Chim. Acta, 204, 223 (1998).

120. R. Forster and D. Diamond, unpublished works.

121. O. Manabe and S. Shinkai, Jpn. Kokai Tokkyo Koho JP 61, 83, 156 (86, 83, 156), 26 Apr. 1986, Appl. 84/205, 990, 29. Sept. 1984, 5 pp.

122. S. Shinkai, S. Mori, H. Koreishi, T. Tsubaki and O. Manabe, J. Am. Chem. Soc., 108 (9), 2409 (1986).

123. M.M. Olmstead, G. Sigel, H. Hope, X. Xu and P.P. Power, J. Am. Chem. Soc., 107 (26), 8087 (1985).

124. B.M. Furphy, J.M. Harrowfield, D.L. Kepert, B.W. Skelton, A.H. White and F.R. Wilner, Inorg. Chem., 26 (25), 423 (1987).

125. S. Shinkai, Pure Appl. Chem., 58 (11), 1523 (1986).

126. S. Ishida and Y. Nakamoto, Netsu Kokasei Jushi, 6 (4), 231 (1985).

127. Y. Kondo, T. Yamamoto, O. Manabe and S. Shinkai, Jpn. Kokai Tokkyo Koho JP 62, 210, 055 [87, 210, 055], 16 Sep. 1987, Appl. 86/54, 322, 11 Mar. 1986, 6 pp.

128. Y. Kondo, T. Yamamoto, O. Manabe and S. Shinkai, Jpn. Kokai Tokkyo Koho JP 63.99, 035 [88, 99, 035], 30 Apr. 1988, Appl. 86/244, 868, 14 Oct. 1986, 4 pp.

129. Y. Kondo, T. Yamamoto, O. Manabe and S. Shinkai, Jpn. Kokai Tokkyo Koho JP 63.99, 031 [88, 99, 031], 30 Apr. 1988, Appl. 86/244, 869, 14 Oct. 1986, 3 pp.

130. S.J. Harris, Eur. Pat. Appl. EP 237,265, 16 Sep. 1987, IE Appl. 596,07 Mar. 1986, 24 pp.

131. K. Kimura, M. Matuso and T. Shono, Chem. Lett., 4, 615 (1988).

132. S. Shinkai, K. Araki and O. Manabe, J. Am. Chem. Soc., 110 (21), 7214 (1988). 
133. S. Shinkai, S. Mori, K. Araki and O. Manabe, Bull Chem. Soc. Jpn., 60(1), 3679 (1987).

134. T. Arimura, M. Kubota, T. Matsuda, O. Manabe and S. Shinkai, Bull. Chem. Soc. Jpn., 62(5), 1674 (1989).

135. S.G. Bott, A.W. Coleman and J.L. Atwood, J. Inclusion Phenom., 5(6), 747 (1987).

136. Y. Kondo, T. Yamamoto, O. Manabe and S. Shinkai, Jpn. Kokai Tokkyo Koho JP 63.277, 240 [88, 277, 240], 15 Nov. 1988, Appl. 87/.113, 094, 09 May 1987, 5 pp.

137. S. Shinkai and O. Manabe, Nippon Kagaku Kaishi, 12, 1917 (1988).

138. M. Halit, D. Oehler, M. Perrin, A. Thozet, R. Perrin, J. Vicens and M. Bourakhouadar, J. Inclusion Phenom., 6(6), 613 (1988).

139. Y. Kondo, T. Yamamoto, O. Manabe and S. Shinkai, Jpn. Kokai Tokkyo Koho JP 63, 197, 544, 16 Aug. 1988, Appl. 86/244, 867, 14 Oct. 1986, 5 pp.

140. S. Shinkai, S. Edamitsu, T. Arimura and O. Manabe, J. Chem. Soc., Chem. Commun., 1988 (24), 1622.

141. S. Shinkai, Y. Otsuka, K. Araki and T. Matsuda, Bull. Chem. Soc. Jpn., 62(12), 4055 (1989).

142. K. Kimura, T. Miura, M. Matsuo and T. Shono, Anal. Chem., 62(14), 1510 (1990).

143. P.D. Beer, A.D. Keafe and M.G.B. Drew, J. Organomet. Chem., 378(3), 437 (1987).

144. F. Corazza, C. Floriani, A. Chiesi-Villa and C. Guastini, J. Chem. Soc., Chem. Commun., 1990 (16), 1083.

145. B.M. Furphy, J.M. Harrowfield, M.I. Ogden, B.W. Skelton, A.H. White and F.R. Wilner, J. Chem. Soc., Dalton Trans., 1989 (11), 2217.

146. P. Zhang, F. Guo and Z. Huang, Huaxue Xuebao, 47 (8), 731 (1989).

147. A.M. Cadogan, D. Diamond, M.R. Smyth, M. Deasy, M.A. Mckervey and S.J. Harris, Analyst (London), 114 (12), 1551 (1989).

148. A.M. Cadogan, D. Diamond, M.R. Smyth, G. Svehla, M.A. Mckervey, E.M. Seward and S.J. Harris, Analyst (London), 115(9), 1207 (1990). 
149. I. Maeda and H. Taniguchi, Jpn. Kokai Tokkyo Koho JP 02, 123, 126 [90, 123, 126], 10 May 1990, Appl. 88/277, 634, 01 Nov. 1988, 3 pp.

150. S. Shinkai, Y. Otsuka, K. Fujimoto and T. Matsuda, Chem. Lett., 1990 (5), 835.

151. E. Nomura, H. Taniguchi, Jpn. Kokai Tokkyo Koho JP 02, 124, 863 [90, 124, 863], 14 May 1990, Appl. 88/277, 633, 01 Nov. 1988, 4 pp.

152. A. Yamada, T. Murase, K. Kitukawa, T. Matsuda and S. Shinkai, Chem. Lett., 1990 (3), 455.

153. V. Boehmer, W. Vogt, H. Goldmann, M.A. Mckervey, M. Owens, S. Cremin and E.M. Collins, J. Org. Chem., 55(9), 2569 (1990).

154. I. Alam and C.D. Gutsche, J. Org. Chem., 55(14), 4487 (1990).

155. Y. Aoyama, Y. Nonaka, Y. Tanaka, H. Toi and H. Ogoshi, J. Chem. Soc., Perkin Trans. II., 1989, (8), 1025.

156. I. Yoshida, S. Fujii, K. Ueno, S. Shinkai and T. Matsuda, Chem. Lett., 1989 (9), 1535.

157. Y. Ishikawa, T. Kunitake, T. Matsuda, T. Otsuka and S. Shinkai, J. Chem. Commun., 1989 (11), 736.

158. E. Nomura, H. Taniguchi and S. Tamura, Chem. Lett., 1989 (7), 1125.

159. C. Floriani, D. Jacoby, A. Chiesi-Villa and C. Guastini, Angew. Chem., 101(10), 1430 (1989).

160. S. Shinkai, H. Kawabata, T. Arimura, H. Satoh and O. Manabe, J. Chem. Soc., Perkin Trans. I., 1989 (5), 1073.

161. T. Nagasaki, S. Shinkai and T. Matsuda, J. Chem. Soc., Perkin Trans. I., 1990 (9), 2617.

162. T. Nagasaki and S. Shinkai, J. Chem. Soc., Perkin Trans. II, 1991 (7), 1063.

163. J.L. Atwood, D.L. Clark, R.K. Juneja, G.W. Orr, K.D. Robinson and R.L. Vincent, J. Am. Chem. Soc., 114(19), 7558 (1992).

164. G. Deng, T. Sakaki, K. Nakashima and S. Shinkai, Chem. Lett., 1992 (7), 1287.

165. S. Shinkai, T. Arimura, T. Nagasaki and T. Matsuda, Jpn. Kokai Tokkyo Koho JP 0491.070 [92, 91, 070], 24 Mar. 1992, Appl. 90/208,849, 06 Aug. 1990, 5 pp.

166. C.D. Gutsche and K.A. See, J. Org. Chem., 57 (16), 4527 (1992). 
167. I. Aoki, T. Sakaki and S. Shinkai, J. Chem. Soc., Chem. Commun., 1992 (9), 730.

168. G. Deng, T. Sakaki, Y. Kawahara and S. Shinkai, Tetrahedron Lett., 33(16), 2163 (1992).

169. M. Conner, V. Janout and S.L. Regen, J. Org. Chem., 57(13), 3744 (1992).

170. T. Aihara, A. Goto, T. Kago, K. Kusakabe and S. Morooka, Sept. Sci. Technol., 27(12), 1655 (1992).

171. Y. Shibutani, K. Yakabe, T. Shono, Y. Yamashoji and M. Tanaka, Anal. Sci., 7, 1671 (1991).

172. I. Aoki, H. Kawabata, K. Nakashima and S. Shinkai, J. Chem. Soc., Chem. Commun., 1991 (24), 1771.

173. T.M. Liang, K.K. Laali, M. Cordero and C. Wesdemiotis, Chem. Res. Synop., 12, 354 (1991).

174. W.I. Iwema-Bakker and D.N. Reinhoudt, New Sep. Chem. Tech. Radioact. Waste, Other Specific Appl. [Proc. Tech. Semin.], 1991; p. 142.

175. I. Yoshida, N. Yamamoto, F. Sagara, K. Ueno, D. Ishii and S. Shinkai, Chem. Lett., 1991 (12), 2105.

176. M. Telting-Diaz, D. Diamond, M.R. Smyth, E.M. Seward and M.A. Mckervey, Electroanalysis (N.Y.), 3(4-5), 371 (1991).

177. J.L. Atwood, G.W. Orr, N.C. Means, F. Hamada, H. Zhang, S. Bott and K.D. Robinson, Inorg. Chem., 31 (4), 603 (1992).

178. G. Goermar, K. Seiffarth, M. Schulz and C. Chachimbombo, J. Prakt. Chem., 333(3), 475 (1991).

179. J.A.J. Brunink, J.R Haak, J.G. Bomer, D.N. Reinhoudt, M.A. Mckervey and S.J. Harris, Anal. Chim. Acta, 254(1-2), 75 (1991).

180. H. Shimizu, K. Iawamoto, K. Fujimoto and S. Shinkai, Chem. Lett., 1991 (12), 2147.

181. T. Arimura, S. Matsumoto, O. Teshima, T. Nagasaki and S. Shinkai, Tetrahedron Lett., 32, 5111 (1991).

182. D. Kraft, R. Cacciapaglia, V. Boehmer, A. Abu-el-Fadi, S. Harkemá, L. Mandolini, D.N. Reinhoudt, W. Verboom and W. Vogt, J. Org. Chem., 57(3), 826 (1992). 
183. J.M. Harrowfield, M.I. Ogden and A.H. White, Aust. J. Chem., 44(9), 1237 (1991).

184. J.M. Harrowfield, M.I. Ogden and A.H. White, J. Chem. Soc., Dalton Trans., 1991 (10), 2625.

185. J.A.J. Brunink, J.G. Bormer, J.F.J. Engbersen, W. Verboom and D.N. Reinhoudt, Sens. Actuators, B, 15, 195 (1993).

186. F. Amaud-Neu, G. Barrett, S.J. Harris, M. Owens, M.A. Mckervey, M.J. Schwing-Weill and P. Schwinte, Inorg. Chem., 32(12), 2644 (1993).

187. H. Kawabata and S. Shinkai, Chem. Express, 8(9), 765 (1993).

188. M. Sato, I. Yoshida and S. Shinkai, Chem. Lett., 1993 (7), 1261.

189. W.H. Chan, K.K. Shiu and X.H. Gu, Analyst, 118(7), 863 (1993).

190. M. Careri, A. Casnati, A. Guarinoni, A. Mangia, G. Mori, A. Pochini and R. Ungaro, Anal. Chem., 65 (21), 3156 (1993).

191. A. Varnek and G. Wipff, J. Phys. Chem., 97, 10840 (1993).

192. T. Sakaki, T. Harada, G. Deng, H. Kawabata , Y. Kawahara and S. Shinkai, J. Inclusion Phenom., Mol. Recognit. Chem., 14(3-4), 285 (1992).

193. K. Araki, N. Hashimoto, H. Otsuka, T. Nagasaki and S. Shinkai, Chem. Lett., 1993 (5), 829.

194. J.C.G. Bünzli, P. Froidevaux and J.M. Harrowfield, Inorg. Chem., 32(15), 3306 (1993).

195. K. Cunningham, G. Svehla, S.J. Harris and M.A. Mckervey, Analyst, 118(4), 341 (1993).

196. K.M. O'Connor, G. Svehla, S.J. Harris and M.A. Mckervey, Anal. Proc., 30(3), 137 (1993).

197. R. Ludwig, K. Inoue and T. Yamato, Solvent Extn. Ion. Exch., 11(12), 311 (1993).

198. J.A.J. Brunink, W. Verboom, J.F.J. Engbersen, S. Harkema and D,N. Reinhoudt, Rec. Trav. Chim. Pays Bas, 111(12), 511 (1992).

199. K. Odashima, K. Yagi, K. Tohda and Y. Umezawa, Anal. Chem., 65(8), 1074 (1993).

200. W. Xu, J.P. Rourke, J.J. Vittal and R.J. Puddephatt, J. Chem. Soc., Chem. Commun., 1993 (2), 145. 
201. G. Arena, R. Cali, G. Lombardo, E. Rizzarelli, D. Sciotti, R. Ungaro and A. Casnati, Supramol. Chem., 1(1), 19 (1992).

202. P.D. Beer, J.P. Martin and M.G.B. Drew, Tetrahedron, 48, 9917 (1992).

203. P.L.M. Cobben, R.J.M. Egberink, J.G. Bomer, P. Bergveld, W. Verboom and D.N. Reinhoudt, J. Am. Chem. Soc., 114(26), 10573 (1992).

204. S.J. Harris, M.A. Mckervey, G. Svehla and D. Diamond, Eur. Pat. Appl. EP 490, 631, 17 June 1992, Appl. 4, 444, 10 Dec. 1990, 36 pp.

205. J.D. Glennon, K.M. O'Connor, S. Brijaranai, K. Manley, S.J. Harris and M.A. Mckervey, Anal. Lett., 26(1), 153 (1993).

206. F. Arnaud-Neu, V. Boehmer, L. Guerra, M.A. Mckervey, E.F. Paulus, A. Rodriguez, M.J. Schwing-Weill, M.S. Tabatabai and W. Vogt, J. Phys. Org. Chem., 5(8), 47 (1992).

207. S.K. Chang, M.J. Jang, S.Y. Han, J.H. Lee, M.H. Kang and K.T. No, Chem. Lett., 1992 (10), 1937.

208. E. Nomura, H. Taniguchi, K. Kawaguchi and Y. Otsujni, J. Org. Chem., 58 (17), 4709 (1993).

209. N. Sato and S. Shinkai, J. Chem. Soc., Perkin Trans. II, 1993 (4), 621.

210. S. Shinkai and F. Ooseto, Jpn. Kokai Tokkyo Koho JP 05 70,413 [93, 70, 413], 23 March 1993, Appl. 91/261,218,12 Sep. 1991, 11 pp.

211. C. Perez-Jimenez, S.J. Harris and D. Diamond, J. Chem. Soc., Chem. Commun., 1993 (5), 480.

212. Y. Kubo, S. Hamaguchi, A. Niimi, K. Yoshida and S. Tokita, J. Chem. Soc., Chem. Commun., 1993 (3), 305.

213. R. Seangprasertkij, Z. Asfari, F. Arnaud-Neu, J. Weiss and J. Vicens, J. Inclusion Phenom., Mol. Recognit. Chem., 14 (2), 141 (1992).

214. P.D. Beer, M.G.B. Drew, C. Hazlewood, D. Hesek, J. Hodacova and S.E. Stokes, J. Chem. Soc., Chem. Commun., 1993 (3), 229.

215. W. Xu, J.J. Vittal and R.J. Puddephatt, J. Am. Chem. Soc., 115 (14), 6456 (1993).

216. I. Yoshida, K. Koyabu, M. Nishida, F. Sagara, D. Ishii and S. Shinkai, Anal. Sci., 10 (2), 353 (1994). 
217. J.L. Atwood, G.A. Koutsantonis and C.L. Raston, Nature (London), 368, 229 (1994).

218. X. Shi, S. Ding, Y. Yang, D. Xu, Y. Chen and Z. Huang, Wujl Hucxue Xuebao, 9 (4), 423 (1993).

219. Y. Ueda, I. Fujiwara, K.I. Tomita, Z. Asfari and J. Vicens, J. Inclusion Phenom., Mol. Recognit. Chem., 15 (4), 341 (1993).

220. N. Yamamoto, M. Nishida, I. Yoshida, F. Sagara, K. Ueno, D. Ishii and S. Shinkai, Bunseki Kagaku, 43 (1), 49 (1994).

221. J.P. Scharff, M. Mahjoubi and R. Perrin, New J. Chem., 17 (12), 793 (1993).

222. F. Inokurchi, Y. Shiomi, H. Kawabata, T. Sakaki and S. Shinkai, Chem. Lett., 1993 (9), 1595.

223. G. Deng, T. Sakaki, Y. Kawahara and S. Shinkai, Supramol. Chem., 2 (1), 71 (1993).

224. J. Soedarsono, M. Burgard, Z. Asfari and J. Vicens, New J. Chem., 17, 601 (1993).

225. A. Odani, Kagaku (Kyoto), 48 (12), 872 (1993).

226. K. Seangprasertkij, Z. Asfari and J. Vicens, J. Inclusion Phenom., Mol. Recognit. Chem., 17 (2), 111 (1994).

227. P.D. Beer, Z. Chang, AJ. Goulden, A. Grieve, D. Hesek, F. Szemes and T. Wear, J. Chem. Soc., Chem. Commun., 1994 (10), 1269.

228. A.M. Reichwein, W. Verboom, S. Harkema, A.L. Spek and D.N. Reinhoudt, J. Chem. Soc., Perkin Trans. II, 1994 (6), 1167.

229. S.Y. Han, M.H. Kang, Y.E. Jung and S.K. Chang, J. Chem. Soc., Perkin Trans. II, 1994 (4), 835.

230. W. Huang and J. Liu, Chin. J. Chem., 11 (4), 370 (1993).

231. E. Malinowska, Z. Brzozka, K. Kasiura, R.J.M. Egberink and D.N. Reinhoudt, Anal. Chim. Acta, 298 (2), 253 (1994).

232. F. Arnaud-Neu, G. Barrett, S. Fanni, D. Marrs, W. McGregor, MA. Mckervey, M.J. Schwing-Weill, V. Vetrogon and S. Wechsler, J. Chem. Soc., Perkin Trans. $I I, 1995$ (3), 453.

233. X. Shi, S. Ding, Y. Yang, S. Li, Y. Xu and Y. Chen, Wuji Huaxue Xuebao, 10 (4), 430 (1994). 
234. H. Iki, T. Kikuchi, H. Tsuzuki and S. Shinkai, J. Inclusion Phenom., Mol. Recognit. Chem., 19 (1-4), 227 (1994).

235. X. Delaigue, M.W. Hosseini, N. Kyritsakas, A. Decain and J. Fischer, J. Chem. Soc., Chem. Commun., 1995 (6), 609.

236. L. Dei, A. Casnati, P.L. Nostro and P. Baglioni, Langmuir, 11 (4), 1268 (1995).

237. T. Sakaki, T. Harada, Y. Kawahara and S. Shinkai, J. Inclusion Phenom., Mol. Recognit. Chem., 17 (4), 377 (1994).

238. F. Arnaud-Neu, S. Fanni, L. Guerra, W. McGregor, K. Ziat, M.J. Schwing-Weill, B. Barrett, M.A. Mckervey, D. Marrs and E.M. Seward, J. Chem. Soc., Perkin Trans. II, 1995 (1), 113.

239. P.D. Beer, Z. Chen, M.G.B. Drew and P.A. Gale, J. Chem. Soc., Chem. Commun., 1994 (19), 2207.

240. F. Davis, L. O'Toole, R. Short and C.J.M. Stirling, Langmuir, 12, 1892 (1996).

241. T. Suzuki, K. Nakashima and S. Shinkai, Tetrahedron Lett., 36, 244 (1995).

242. R.M. Williams, J.M. Zwier and J.W. Verhoeven, J. Am. Chem. Soc., 116, 6965 (1994).

243. I. Hall, G.P. Nicholson, T.J. Piper, D.M. Taylor, G. Williams and D.K. Williams, Radiochim. Acta, 69 (4), 225 (1995).

244. B.H. Huisman, D.M. Rudkevich, F.C.J.M. Van Veggel and D.M. Reinhoudt, J. Am. Chem. Soc., 118, 3523 (1996).

245. K. Ohto, E. Murakami, K. Shiratsuchi, K. Inoue and M. Iwasaki, Chem. Lett., 1996 (2), 173.

246. Y.K. Agrawal and S. Mallika, Analyst (Cambridge), 120 (11), 2759 (1995).

247. Y.K. Agrawal and S. Mallika, J. Radional. Nucl. Chem., 198 (2), 349 (1995).

248. F.J. Parlevliet, A. Olivier, W.G.J. deLange, P.C.J. Kamer, H. Kooijman, A.L. Spek and P.W.N.M. Van Leeuwen, Chem. Commun. - (Cambridge), 1996 (5), 583.

249. T. Grady, S. Maskula, D. Diamond, D.J. Marrs, M.A. Mckervey and P. O'Hagan, Anal. Proc., 32 (11), 471 (1995). 
250. A.T. Yordanov, J.T. Mague and D.M. Roundhill, Inorg. Chem. Acta, 2441 (1-2), 441 (1995).

251. Y. Koide, H. Sato, H. Shosenji and K. Yamada, Bull. Chem. Soc. Jpn., 69, 315 (1996).

252. A. Zanotti-Gerosa, E. Solari, I. Giannini, C. Floriani, A. Chiesi-Villa and C. Rizzoli, J. Chem. Soc., Chem. Commun., 1996 (2), 123.

253. Z.L. Zhong, C. Tang, C. Wu and Y. Chen, J. Chem. Soc., Chem. Commun., 1995 (17), 1737.

254. L. Zhang, L.A. Godinez, T. Lu, G.W. Gokel and A.E. Kaifer, Angew. Chem. Int. Ed. Eng., 34 (2), 235 (1995).

255. J.E. Bollinger, J.K. Moran, E.M. Georgiev and D.M. Roundhill, Supramol. Chem., 4 (3), 173 (1994).

256. K. Kimura and O. Ikagaku, Kenkyo Zaidan Kenkyu Hokoku, 12, 69 (1993).

257. T. Laiki, K. Goto, N. Tokitoh, M. Goto and R. Okazadi, Tetrahedron Lett., 37 (23), 4039 (1996).

258. A.F.D. deNamor, F.J.S. Velarde and M.C. Cubaleiro, J. Chem. Soc., Faraday Trans., 93 (10), 1731 (1996).

259. S. Shinkai, K. Fujimoto, M. Ogata and Y. Harada, Jpn. Kokai Tokkyo Koho JP. 08, 127, 561 [96, 127, 561], 21 May, 1996, Appl. 94/267, 142, 31 Oct. 1994, 6 pp.

260. H. Konishi, T. Tamura, H. Ohkubo, K. Kobayashi and O. Morikawa, Chem. Lett., 1996 (8), 685.

261. A. Ikeda and S. Shinkai, Chem. Lett., 1996 (9), 803.

262. A. Varnek, C. Sirlin and G. Wipff, NATO ASI Ser., Ser. C, 480, 67 (1996).

263. M.A. Mckervey, M. Schwing-Weill and F. Arnaud-Neu, Compr. Supramol. Chem., 1, 537 (1996).

264. C.L. Raston, Compr. Supramol. Chem., 1, 777 (1996).

265. T. Mckittrick, D. Diamond, D.J. Marrs, P. O'Hagan and MA. Mckervey, Talanta, 43, 1145 (1996).

266. H.C.Y. Bettega, J.C. Moutet, G. Ulrich and R. Ziessel, J. Electroanal. Chem., 406, 247 (1996). 
267. P.S.H. Wong, X. Yu and D.Y. Dearden, Inorg. Chim. Acta, 246, 259 (1996).

268. P.D. Beer, M.G.B. Drew, P.B. Leason and M.T. Ogden, Inorg. Chim. Acta, 246, 133 (1996).

269. H. Deligoz and M. Yilmaz, Synth. React. Inorg. Met.-Org. Chem., 26 (6), 943 (1996).

270. U.S. Vural and M. Ersoz, J. Appl. Polym. Sci., 61 (8), 1273 (1996).

271. A.M. Nechifor, A.P. Philipse, F. deJong, J.P.M. Van Duynhoven, R.J.M. Egberink and D.N. Reinhoudt, Langmuir, 12 (16), 3844 (1996).

272. T. Takeuchi, J. Chu and T. Miwa, Kuromatogurafi, 17 (2), 142 (1996).

273. N. Muzet, G. Wipff, A. Casnati, L. Domiano, R. Ungaro and F. Ugozzoli, J. Chem. Soc., Perkin Trans. II, 1996, 1065.

274. W. Wroblewski and Z. Brzozka, Anal. Chim. Acta, 326, 163 (1996).

275. P.D. Beer, M.G.B. Drew, A. Grieve, M. Kan, P.B. Leason, G. Nicholson, M.I. Ogden and G. Williams, Chem. Commun., 1996 (10), 1117.

276. M. Nishida, I. Yoshida, D. Isliii and S. Shinkai, Nippon Kagaku Kaishi, 5, 449 (1996). 